\title{
WAVEPACKETS IN INHOMOGENEOUS PERIODIC MEDIA: PROPAGATION THROUGH A ONE-DIMENSIONAL BAND CROSSING
}

\author{
ALEXANDER WATSON AND MICHAEL I. WEINSTEIN
}

\begin{abstract}
We consider a model of an electron in a crystal moving under the influence of an external electric field: Schrödinger's equation in one spatial dimension with a potential which is the sum of a periodic function $V$ and a smooth function $W$. We assume that the period of $V$ is much shorter than the scale of variation of $W$ and denote the ratio of these scales by $\epsilon$. We consider the dynamics of semiclassical wavepacket asymptotic (in the limit $\epsilon \downarrow 0$ ) solutions which are spectrally localized near to a crossing of two Bloch band dispersion functions of the periodic operator $-\frac{1}{2} \partial_{z}^{2}+V(z)$. We show that the dynamics is qualitatively different from the case where bands are well-separated: at the time the wavepacket is incident on the band crossing, a second wavepacket is 'excited' which has opposite group velocity to the incident wavepacket. We then show that our result is consistent with the solution of a 'Landau-Zener'-type model.
\end{abstract}

\section{INTRODUCTION}

In this work we study the non-dimensionalized, semi-classically scaled, time-dependent Schrödinger equation for $\psi^{\epsilon}(x, t): \mathbb{R} \times[0, \infty) \rightarrow \mathbb{C}$ :

$$
\begin{aligned}
& i \epsilon \partial_{t} \psi^{\epsilon}=-\frac{1}{2} \epsilon^{2} \partial_{x}^{2} \psi^{\epsilon}+V\left(\frac{x}{\epsilon}\right) \psi^{\epsilon}+W(x) \psi^{\epsilon} \equiv H^{\epsilon} \psi^{\epsilon} \\
& \psi^{\epsilon}(x, 0)=\psi_{0}^{\epsilon}(x) .
\end{aligned}
$$

Here, $\epsilon$ is a positive real parameter which we assume to be small. We assume throughout that the function $V$ is smooth and 1-periodic so that:

$$
V(z+1)=V(z) \text { for all } z \in \mathbb{R},
$$

and that $W$ is smooth with all derivatives uniformly bounded (this assumption may be relaxed; see Remark 1.2 of [47]). Equation (1.1] is the independent-particle approximation in condensed matter physics $[2]$ for the dynamics of an electron in a crystal described by periodic potential $V$, under the influence of an external electric field generated by a 'slowly varying' potential $W$.

Let $E_{n}$ denote the $n$th Bloch band dispersion function of the periodic operator $-\frac{1}{2} \partial_{z}^{2}+V(z)$. It is known that 6,47 for any uniformly isolated, or non-degenerate, band $E_{n}$ (see Figure 1) there exists a family of explicit asymptotic solutions of 1.1 known as semiclassical wavepackets which, for any fixed positive integer $N$, approximate exact solutions up to 'Ehrenfest time' $t \sim \ln 1 / \epsilon$ up to errors of order $\epsilon^{N}$ in $L_{x}^{2}$. The center of mass and average quasi-momentum of these solutions evolve (up to errors of size $o(1)$ ) along classical trajectories generated by the 'Bloch band' Hamiltonian $\mathcal{H}_{n}:=E_{n}(p)+W(q)$. We refer to such an asymptotic solution as a wavepacket associated with the band $E_{n}$. The 'Ehrenfest' time-scale of validity of the 


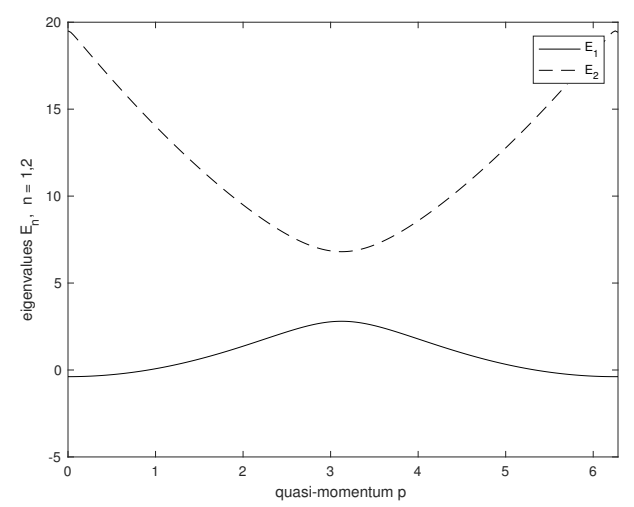

Figure 1. Plot of the two lowest Bloch band dispersion functions $E_{1}(p), E_{2}(p)$ for the 1-periodic potential $V(z)=4 \cos (2 \pi z)$. Both bands are isolated from each other and all other bands: for all $p \in[0,2 \pi], G\left(E_{2}(p)\right)>0$ and $G\left(E_{1}(p)\right)>0$ where $G\left(E_{n}(p)\right)$ is the spectral band gap function (2.8). Consequently, the maps $p \mapsto E_{1}(p), E_{2}(p)$ are smooth.

asymptotics is known to be the general limiting time-scale of validity of wavepacket, or coherent state, approximations 41]. These results generalize to $d$-dimensional analogs of (1.1) 47, 6, see also 24, 25, 39].

In this work we consider the following question concerning the dynamics of wavepackets in a situation where two Bloch bands are not isolated:

Problem. Consider equation (1.1) with initial conditions given by a wavepacket associated with a band $E_{n}$, for which the classical trajectories associated with $\mathcal{H}_{n}$ pass through a point in phase space, $\left(q^{*}, p^{*}\right)$, where the Bloch band $E_{n}$ is degenerate; see Figure 2. How are the dynamics different from the isolated band case?

More precisely, suppose that two bands $E_{n}(p), E_{n+1}(p)$ touch at a quasi-momentum $p^{*}$ in the Brillouin zone, but are otherwise non-degenerate in a neighborhood of $p^{*}$ (see Figure 2). We study a wavepacket associated with the band $E_{n}$ initially localized in phase space on a classical trajectory $(q(t), p(t))$ generated by $\mathcal{H}_{n}$ which encounters the crossing after some finite time $t^{*}$ : for some $t^{*}>0, \lim _{t \uparrow t^{*}} p(t)=p^{*} 1$

We now give a rough statement of our main results; a more precise statement is given in Section 2. Assume that an 'incident' wavepacket is driven through the crossing so that $\lim _{t \uparrow t^{*}} \dot{p}(t)=\lim _{t \uparrow t^{*}} \partial_{q} W(q(t)) \neq 0$. For a precise set up, see the Band Crossing Scenario (Property 3.7). Our main results address:

(1) Quantifying the breakdown of 'single-band' description as $t \uparrow t^{*}$; Theorem 3.12 Fix any positive integer, $N$. For $t \ll t^{*}$, the solution of (1.1) can be represented as a wavepacket associated with the band $E_{n}$ with errors which are $O\left((\sqrt{\epsilon})^{N}\right)$ in $L^{2}(\mathbb{R})$. As $t \uparrow t^{*}$, this 'single-band' description fails to capture the dynamics of the PDE to any order in $\sqrt{\epsilon}$

\footnotetext{
${ }^{1}$ The scenario we consider is non-generic: for generic periodic potentials in one spatial dimension, all bands are isolated 40, 33, 42. Non-trivial periodic potentials exhibiting band crossings may be explicitly displayed (see Section 3.2.
} 


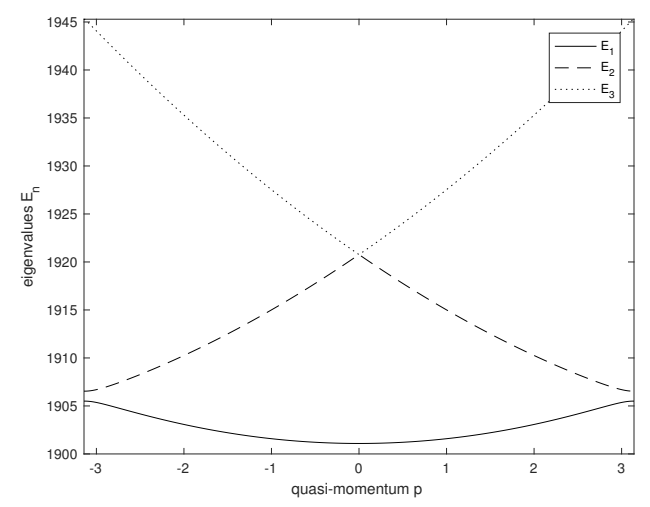

Figure 2. Plot of the three lowest Bloch band dispersion functions for $V(z)=\wp_{1 / 2, i \omega^{\prime}}\left(z+i \omega^{\prime}\right)$, the 'one-gap' potential (see Example 3.5, with $\omega^{\prime}=.8$. The band $E_{1}(p)$ is isolated over the whole Brillouin zone $[-\pi, \pi]$, but the bands $E_{2}(p), E_{3}(p)$ are degenerate at $p=0$. For this choice of potential, for all integers $n \geq 2$ the band $E_{n}(p)$ is degenerate with the band $E_{n+1}(p)$ at either $p=0$ or $p=\pi$. The one and only gap is shown.

higher than order $(\sqrt{\epsilon})^{0}=1$, since it does not incorporate an excited wave associated with the band $E_{n+1}$ whose norm grows to be of the order $\sqrt{\epsilon}$ as $t$ approaches $t^{*}$ on the non-adiabatic time-scale $s=\left(t-t^{*}\right) / \sqrt{\epsilon}$.

(2) Coupling of degenerate bands and excitation of a reflected wavepacket; Theorem 3.20 For $t \sim t^{*}$ and for $t \gg t^{*}$ the solution of (1.1) is well-approximated by the sum of two semiclassical wavepackets: a 'transmitted' wavepacket associated with the band $E_{n+1}$ with $L^{2}$-norm of order 1 and a 'reflected' wavepacket associated with the band $E_{n}$ with $L^{2}$-norm of order $\sqrt{\epsilon}$ (Figure 3). The size of the error terms is $o(\sqrt{\epsilon})$ in $L^{2}(\mathbb{R})$. The expansion is constructed via a rigorous matched-asymptotic analysis in which the 'transmitted' and 'reflected' wave-packets evolve on the emergent non-adiabatic time-scale $s=\left(t-t^{*}\right) / \sqrt{\epsilon}$.

In Appendix A we show that these results are consistent with the solution of an appropriate 'Landau-Zener'-type model.

The proofs of Theorems 3.12 and 3.20 rely on the existence of smooth continuations of the Bloch band dispersion functions $E_{n}, E_{n+1}$ through the crossing point $p^{*}$; see Property 3.1 and Figure 4. Such continuations always exist in one spatial dimension and more generally at arbitrary codimension 1 eigenvalue band crossings (see Theorem 3.2 and 27] for the general case). The key tools in our proof are the method of matched asymptotic expansions (following Hagedorn [27]) combined with the basic result of Carles and Sparber [6] on semiclassical wavepacket solutions in the presence of a periodic background potential.

Our proof does not readily generalize to cases where no smooth continuation of eigenvalue bands exists; for example at 'conical', or 'Dirac', points which are codimension 2 eigenvalue band crossings $16,17,14,15,13,12$. The dynamics of semiclassical wavepackets at such crossings was studied by Hagedorn in the context 


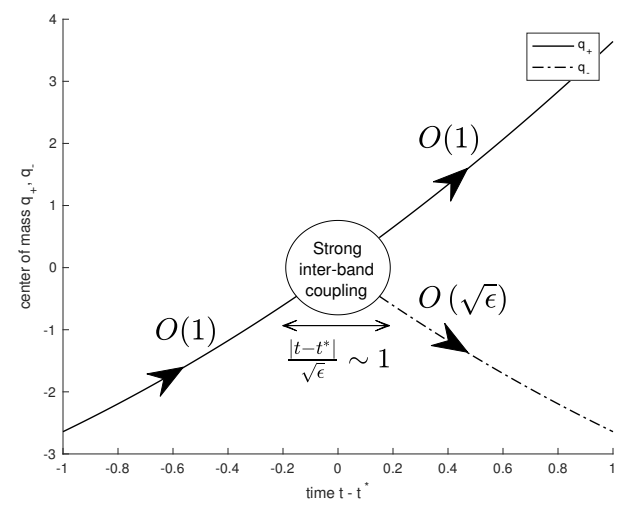

Figure 3. Schematic of center of mass location versus time of the 'incident/transmitted' wavepacket $q_{+}(t)$ and the 'excited/reflected' wavepacket $q_{-}(t)$, which satisfy (3.16) and (3.35). As $t$ approaches $t^{*}$ the average quasi-momentum of the incident wavepacket is degenerate, i.e. $p_{+}\left(t^{*}\right)=p^{*}$ where $E_{+}\left(p^{*}\right)=E_{-}\left(p^{*}\right)$. Interband coupling is non-negligible, occurs over the emergent nonadiabatic time-scale $s:=\frac{t-t^{*}}{\sqrt{\epsilon}}=\mathcal{O}(1)$ and excites a second wavepacket. The size in $L^{2}$ of the 'excited' wavepacket is smaller than that of the 'incident' wavepacket by a factor of $\sqrt{\epsilon}$ and proportional to the 'coupling coefficient' $\left\langle\chi_{-}\left(\cdot ; p^{*}\right) \mid \partial_{p} \chi_{+}\left(\cdot ; p^{*}\right)\right\rangle$. Here $E_{ \pm}(p), \chi_{ \pm}(z ; p)$ refer to the smooth continuations of the band eigenpairs $E_{n}(p), E_{n+1}(p), \chi_{n}(z ; p), \chi_{n+1}(z ; p)$ through the crossing (see Property 3.1 and Figure 44. Such continuations always exist at one-dimensional band crossings (Theorem 3.2.

of the Born-Oppenheimer approximation of molecular physics 27]. Adapting his methods to the present context is the subject of ongoing work. A model of the dynamics at a 'conical' codimension 2 Bloch band degeneracy was derived in [23].

Quantum dynamics at an eigenvalue band crossing was studied by Landau [34 and Zener [49] in the 1930s. Their simplified model, involving an explicitly timedependent Hamiltonian, captures many of the essential features of the dynamics in a neighborhood of the crossing.

For a review of their work, see 37. A rigorous proof of their main result, the 'Landau-Zener' formula for the probability of an inter-band transition, was given by Hagedorn 26 for the case of an 'avoided' crossing. Validity of the formula for true crossings was then proved by Joye 32]. In Appendix A we show that our main theorem (Theorem 3.20 is consistent with the solution of an appropriate 'Landau-Zener'-type simplified model of the present problem.

The propagation of Wigner measures through crossings (both true and avoided) in the context of the Born-Oppenheimer approximation has been studied by FermanianKammerer, Lasser, and others [18, 19, 35, 21, 7, 20, 8, 22. The dynamics of semiclassical wavepackets at an avoided crossing in this context was studied by Hagedorn and Joye 28. A discussion of crossing phenomena from the perspective 


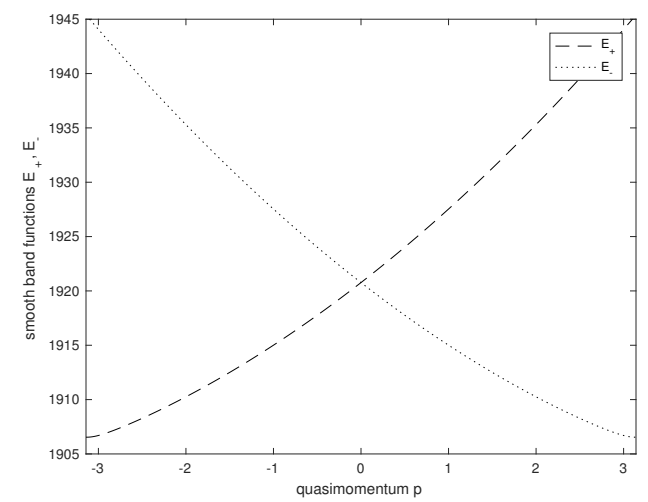

Figure 4. Plot of the maps $E_{+}(p), E_{-}(p)$ defined by (3.3) with $n=2$ and where $E_{2}(p), E_{3}(p)$ are the second and third lowest Bloch band dispersion functions when $V(z)=\wp_{1 / 2, i \omega^{\prime}}\left(z+i \omega^{\prime}\right)$, the 'onegap' potential with $\omega^{\prime}=.8$. The lowest three Bloch bands of this potential are shown in Figure 2.

of normal forms and microlocal analysis was given by Colin de Verdiere et al. 45 , 43,44 .

\subsection{Notation.}

- We introduce a natural set of function spaces which encode both smoothness and spatial decay. For every $l \in \mathbb{N}$ :

$$
\Sigma^{l}(\mathbb{R}):=\left\{f \in L^{2}(\mathbb{R}):\|f\|_{\Sigma^{l}}:=\sum_{|\alpha|+|\beta| \leq l}\left\|y^{\alpha}\left(-i \partial_{y}\right)^{\beta} f(y)\right\|_{L_{y}^{2}}<\infty\right\} .
$$

- The space of Schwartz functions $\mathcal{S}(\mathbb{R})$ is the space of functions defined as:

$$
\mathcal{S}(\mathbb{R}):=\cap_{l \in \mathbb{N}} \Sigma^{l}(\mathbb{R}) .
$$

- We will refer throughout to the space of $L^{2}$-integrable functions which are 1-periodic:

$$
L_{\text {per }}^{2}:=\left\{f \in L_{\text {loc }}^{2}(\mathbb{R}): f(z+1)=f(z) \text { at almost every } z \in \mathbb{R}\right\} .
$$

- For functions of period 1 , the Brillouin zone $\mathcal{B}$ may be chosen to be any real interval of length $2 \pi$. To arrange that the band degeneracy occurring at quasi-momentum $p^{*}=\pi$ is located at an interior point of $\mathcal{B}$, we fix $\mathcal{B}:=[0,2 \pi]$.

- Conventions for $L^{2}$-inner products and induced norms:

$$
\langle f \mid g\rangle_{L^{2}(\mathcal{D})}:=\int_{\mathcal{D}} \overline{f(x)} g(x) \mathrm{d} x, \quad\|f\|_{L^{2}(\mathcal{D})}:=\langle f \mid f\rangle_{L^{2}(\mathcal{D})}^{1 / 2}
$$

For brevity, when $\mathcal{D}=\mathbb{R}$ we omit the domain of integration:

$$
\langle f \mid g\rangle_{L^{2}}:=\int_{\mathbb{R}} \overline{f(x)} g(x) \mathrm{d} x, \quad\|f\|_{L^{2}}:=\langle f \mid f\rangle_{L^{2}}^{1 / 2},
$$


and when $\mathcal{D}=[0,1]$ we omit all subscripts:

$$
\langle f \mid g\rangle:=\int_{[0,1]} \overline{f(x)} g(x) \mathrm{d} x, \quad\|f\|:=\langle f \mid f\rangle^{1 / 2} .
$$

- We make the following convention for the Fourier transform and its inverse:

$$
\mathcal{F}_{x}\{f(x)\}(\xi):=\int_{-\infty}^{\infty} e^{-2 \pi i \xi x} f(x) \mathrm{d} x, \quad \mathcal{F}_{\xi}^{-1}\{g\}(x):=\int_{-\infty}^{\infty} e^{2 \pi i \xi x} g(\xi) \mathrm{d} \xi .
$$

Acknowledgements. The authors wish to thank George Hagedorn, Jianfeng Lu, and Christof Sparber for stimulating discussions. This research was supported in part by National Science Foundation Grant Nos. DMS-1412560, DMS-1620418 and Simons Foundation Math + X Investigator Award \#376319 (Michael I. Weinstein).

\section{Review of Floquet-Bloch theory and the isolated Band theory of WAVEPACKETS}

2.1. Floquet-Bloch theory. In order to state our results we require some background on the spectral theory of the Schrödinger operator:

$$
H:=-\frac{1}{2} \partial_{z}^{2}+V(z)
$$

where $V$ is 1-periodic (see 33,40 for proofs and details). Consider the family of self-adjoint eigenvalue problems parameterized by the real parameter $p$ :

$$
\begin{aligned}
& H \Phi(z ; p)=E(p) \Phi(z ; p) \\
& \Phi(z+1 ; p)=e^{i p} \Phi(z ; p) \text { for all } z \in \mathbb{R} .
\end{aligned}
$$

Because of the explicit $2 \pi$-periodicity of the boundary condition, there is no loss of generality in restricting our attention to $p \in \mathcal{B}$, where $\mathcal{B}$ is any real interval of length $2 \pi . \mathcal{B}$ is usually fixed to be $[-\pi, \pi]$ or $[0,2 \pi]$ and referred to as the Brillouin zone. The eigenvalue problem (2.2) is equivalent (by the transformation $\left.\Phi(z ; p)=e^{i p z} \chi(z ; p)\right)$ to the family of self-adjoint eigenvalue problems with 1periodic boundary conditions:

$$
\begin{aligned}
& H(p) \chi(z ; p)=E(p) \chi(z ; p) \\
& \chi(z+1 ; p)=\chi(z ; p) \text { for all } z \in \mathbb{R} \\
& H(p):=\frac{1}{2}\left(p-i \partial_{z}\right)^{2}+V(z) .
\end{aligned}
$$

For fixed $p$, the spectrum of the equivalent operators 2.2 and 2.3 is real and discrete and the eigenvalues can be ordered with multiplicity:

$$
E_{1}(p) \leq E_{2}(p) \leq \ldots \leq E_{n}(p) \leq \ldots .
$$

The maps $p \mapsto E_{n}(p)$, for $p$ varying over $\mathcal{B}$, are known as the spectral band functions. Their graphs are Lipschitz continuous and are called the dispersion curves of $H$. The set of all dispersion curves as $p$ varies over $\mathcal{B}$ is called the band structure of $H$ (2.1). The associated normalized eigenfunctions $\chi_{n}(z ; p)$ of $(2.3)$ are a basis of the space:

$$
L_{\text {per }}^{2}:=\left\{f \in L_{l o c}^{2}: f(z+1)=f(z) \text { at almost every } z \in \mathbb{R}\right\},
$$

and any function in $L^{2}(\mathbb{R})$ may be expressed as a superposition of Bloch waves:

$$
\left\{\Phi_{n}(z ; p)=e^{i p z} \chi_{n}(z ; p): n \in \mathbb{N}, p \in \mathcal{B}\right\} .
$$


The $L^{2}$-spectrum of the operator $(2.1)$ is the union of the real intervals swept out by the spectral band functions $E_{n}(p)$ :

$$
\sigma(H)_{L^{2}\left(\mathbb{R}^{d}\right)}=\cup_{n \in \mathbb{R}}\left\{E_{n}(p): p \in \mathcal{B}\right\} .
$$

We define a measure of the spectral gap or separation at quasimomentum $p \in \mathcal{B}$ between $E_{n}$ and all other spectral band functions satisfying (2.3):

$$
G\left(E_{n}(p)\right):=\min _{m \neq n}\left|E_{n}(p)-E_{m}(p)\right| .
$$

We make the following definitions:

Definition 2.1. Let $E_{n}(p)$ denote an eigenvalue band of either of the equivalent eigenvalue problems 2.2, 2.3) and let $\tilde{p} \in \mathcal{B}$. If:

$$
G\left(E_{n}(\tilde{p})\right)>0,
$$

then we will say that $E_{n}(p)$ is isolated at $\tilde{p}$. If:

$$
G\left(E_{n}(\tilde{p})\right)=0,
$$

then we will say that $E_{n}(p)$ is involved in a Bloch band degeneracy or band crossing at $\tilde{p}$.

\subsection{Isolated band theory.}

Property 2.2 (Isolated Band Property). Let $E_{n}$ denote a band dispersion function satisfying (2.3) for $p \in \mathcal{B}$. Let $t_{0}<t_{1} \leq \infty$ and $q_{0}, p_{0} \in \mathbb{R} \times \mathcal{B}$ be such that the equations of motion of the classical Hamiltonian $\mathcal{H}_{n}(p, q):=E_{n}(p)+W(q)$ :

$$
\begin{array}{ll}
\dot{q}(t)=\partial_{p} E_{n}(p(t)), & \dot{p}(t)=-\partial_{q} W(q(t)) \\
q\left(t_{0}\right)=q_{0} & p\left(t_{0}\right)=p_{0}
\end{array}
$$

have a unique smooth solution $(q(t), p(t))$ for $t \in\left[t_{0}, t_{1}\right)$ such that $E_{n}$ is isolated along the trajectory $(q(t), p(t))$ for $t \in\left[t_{0}, t_{1}\right)$; i.e:

$$
M\left(t_{0}, t_{1}\right):=\inf _{t \in\left[t_{0}, t_{1}\right)} G\left(E_{n}(p(t))\right)>0,
$$

where $G\left(E_{n}(p)\right)$ is defined by 2.8.

For arbitrary constant $S_{0} \in \mathbb{R}$ we let $S(t)$ denote the classical action along the path $(q(t), p(t))$ :

$$
S(t)=S_{0}+\int_{t_{0}}^{t} p\left(t^{\prime}\right) \partial_{p} E_{n}\left(p\left(t^{\prime}\right)\right)-E_{n}\left(p\left(t^{\prime}\right)\right)-W\left(q\left(t^{\prime}\right)\right) \mathrm{d} t^{\prime}
$$

For arbitrary $a_{0}^{0}(y) \in \mathcal{S}(\mathbb{R})$, let $a^{0}(y, t)$ denote the unique solution of Schrödinger's equation with a time-dependent harmonic oscillator Hamiltonian depending parametrically on the classical trajectory $(q(t), p(t))$ with initial data specified at $t_{0}$ by $a_{0}^{0}(y)$ :

$$
\begin{aligned}
& i \partial_{t} a^{0}(y, t)=\mathscr{H}(t) a^{0}(y, t), \\
& \mathscr{H}(t):=\frac{1}{2} \partial_{p}^{2} E_{n}(p(t))\left(-i \partial_{y}\right)^{2}+\frac{1}{2} \partial_{q}^{2} W(q(t)) y^{2}+\partial_{q} W(q(t)) \mathcal{A}_{n}(p(t)), \\
& a^{0}\left(y, t_{0}\right)=a_{0}^{0}(y) .
\end{aligned}
$$

Here, $p \in \mathcal{B} \mapsto \mathcal{A}_{n}(p)$ denotes the $n$-th band Berry connection (see Section 1.1 for conventions regarding inner products and norms):

$$
\mathcal{A}_{n}(p):=i\left\langle\chi_{n}(\cdot ; p)\right)\left|\partial_{p} \chi_{n}(\cdot ; p)\right\rangle \text {. }
$$


Since the $\chi_{n}(z ; p)$ are assumed normalized: for all $p \in \mathbb{R},\left\|\chi_{n}(\cdot, p)\right\|=1$, it follows that $\mathcal{A}_{n}(p)$ is real-valued. The term $\partial_{q} W(q(t)) \mathcal{A}_{n}(p(t))$ in 2.14 depends only on $t$ and leads to an overall phase shift in the solution of 2.14 known as Berry's phase.

Remark 2.3. Given any path $p(t)$ through parameter space which does not selfintersect (i.e. $p\left(t_{1}\right)=p\left(t_{2}\right) \Longrightarrow t_{1}=t_{2}$ for all $\left.t_{1}, t_{2}\right)$ it is possible to choose phases of the eigenfunctions $\chi_{n}(z ; p)$ in such a way that the Berry connection (2.15) is zero when evaluated along the curve $p(t)$ for all $t$. This choice is known as the adiabatic gauge. See Proposition 3.1 of [27], for example. In dimensions greater than 1, the integral of the Berry connection along a closed path through parameter space is not, in general, zero and is physically observable [3].

We now state a mild refinement of the result of Carles-Sparber $[6]$ which we find more directly applicable:

Theorem 2.4 (Order 1 wave-packet). Let $(q(t), p(t))$ denote the classical trajectory generated by the Hamiltonian $\mathcal{H}_{n}(p, q)=E_{n}(p)+W(q)$, where $p \mapsto E_{n}(p)$ denotes the $n^{\text {th }}$ spectral band function for the periodic Schrödinger operator $-\frac{1}{2} \partial_{z}^{2}+V(z)$. Assume that band $E_{n}$ satisfies the Isolated Band Property 2.2 along the trajectory $(q(t), p(t))$ for $t \in\left[t_{0}, t_{1}\right)$, i.e. $M\left(t_{0}, t_{1}\right)>0$; see (2.12).

Let $S(t)$ be as in 2.13) with $S_{0} \in \mathbb{R}$ and $a^{0}(y, t)$ be the unique solution of 2.14) with initial data $a_{0}^{0}(y) \in \mathcal{S}(\mathbb{R})$.

Then, for sufficiently small $\epsilon>0$ the following holds. Let $\psi^{\epsilon}(x, t)$ denote the unique solution of the initial value problem (1.1) with approximate 'Bloch wavepacket' initial data given at $t=t_{0}$ :

$$
\begin{aligned}
& i \epsilon \partial_{t} \psi^{\epsilon}=H^{\epsilon} \psi^{\epsilon} \\
& \psi^{\epsilon}\left(x, t_{0}\right)=\epsilon^{-1 / 4} e^{i S_{0} / \epsilon} e^{i p_{0}\left(x-q_{0}\right) / \epsilon} a_{0}^{0}\left(\frac{x-q_{0}}{\sqrt{\epsilon}}\right) \chi_{n}\left(\frac{x}{\epsilon} ; p_{0}\right) .
\end{aligned}
$$

For $t \in\left[t_{0}, t_{1}\right)$, the solution evolves as a modulated 'Bloch wavepacket' plus a corrector $\eta^{\epsilon}(x, t)$ :

$$
\psi^{\epsilon}(x, t)=\epsilon^{-1 / 4} e^{i S(t) / \epsilon} e^{i p(t)(x-q(t)) / \epsilon} a^{0}\left(\frac{x-q(t)}{\sqrt{\epsilon}}, t\right) \chi_{n}\left(\frac{x}{\epsilon} ; p(t)\right)+\eta^{\epsilon}(x, t)
$$

where the leading order term is of order 1 in $L^{2}(\mathbb{R})$ and the corrector $\eta^{\epsilon}$ satisfies:

$$
\left\|\eta^{\epsilon}(\cdot, t)\right\|_{L^{2}} \leq C e^{c\left(t-t_{0}\right)} \sqrt{\epsilon}, \quad t_{0} \leq t<t_{1} .
$$

The constants $C>0, c>0$ depend on $M\left(t_{0}, t_{1}\right)$ and the initial data specified at $t_{0}$, are independent of $\epsilon$ and do not depend otherwise on $t_{0}$ and $t_{1}$. Moreover, the constant $C$ in $(2.18)$ satisfies $C \uparrow \infty$ as $M\left(t_{0}, t_{1}\right) \downarrow 0$.

In particular, if $M\left(t_{0}, \infty\right)>0$ then

$$
\sup _{t \in\left[t_{0}, \tilde{C} \ln 1 / \epsilon\right]}\left\|\eta^{\epsilon}(\cdot, t)\right\|_{L^{2}}=o(1),
$$

where $\tilde{C}$ is any constant such that $\tilde{C}<\frac{1}{2 c}$.

Remark 2.5. The timescale $t \sim \ln 1 / \epsilon$ is known as 'Ehrenfest time' and is known to be the general limit of applicability of wavepacket, or coherent state, approximations (see [41] and references therein). 
It is convenient at this point to introduce a short-hand notation for the leading order $\left(O(1)\right.$ in $\left.L^{2}\right)$ 'Bloch wavepacket' asymptotic solution associated with the band $E_{n}$ with centering along the classical trajectory $(q(t), p(t))$ and envelope function $a^{0}(y, t)$ 2.17):

$$
\begin{aligned}
& \mathrm{WP}^{0, \epsilon}\left[S(t), q(t), p(t), a^{0}(y, t), \chi_{n}(z ; p(t))\right](x, t):= \\
& \epsilon^{-1 / 4} e^{i S(t) / \epsilon} e^{i p(t)(x-q(t)) / \epsilon} a^{0}\left(\frac{x-q(t)}{\sqrt{\epsilon}}, t\right) \chi_{n}\left(\frac{x}{\epsilon} ; p(t)\right) .
\end{aligned}
$$

In our analysis we require a refinement of Theorem 1.1 of [47] where it was demonstrated how to compute corrections to the asymptotic solution (2.17) in order to improve the error bound 2.18 by a factor of $\sqrt{\epsilon}$.

For any $a_{0}^{1}(y) \in \mathcal{S}(\mathbb{R})$, let $a^{1}(y, t)$ denote the unique solution of the following inhomogeneous Schrödinger equation with initial data specified at $t_{0}$ by $a_{0}^{1}(y)$ driven by the solution $a^{0}(y, t)$ of 2.14 :

$$
\begin{aligned}
& \left(i \partial_{t}-\mathscr{H}(t)\right) a^{1}(y, t)=\mathscr{I}(t) a^{0}(y, t) \\
& \mathscr{I}(t):=\frac{1}{6} \partial_{p}^{3} E_{n}(p(t))\left(-i \partial_{y}\right)^{3}+\frac{1}{6} \partial_{q}^{3} W(q(t)) y^{3} \\
& +\partial_{q} W(q(t)) \partial_{p} \mathcal{A}_{n}(p(t))\left(-i \partial_{y}\right)+\partial_{q}^{2} W(q(t)) \mathcal{A}_{n}(p(t)) y \\
& a^{1}\left(y, t_{0}\right)=a_{0}^{1}(y) .
\end{aligned}
$$

Again, $\mathcal{A}_{n}(p)$ denotes the Berry connection, displayed in 2.15). We next introduce a convenient short-hand notation for the 'Bloch wavepacket' asymptotic solution associated with the band $E_{n}$ including a first-order in $\sqrt{\epsilon}$ correction to $\mathrm{WP}^{0, \epsilon}$ in 2.20 :

$$
\begin{aligned}
& \mathrm{WP}^{1, \epsilon}\left[S(t), q(t), p(t), a^{0}(y, t), a^{1}(y, t), \chi_{n}(z ; p(t))\right](x, t):= \\
& \epsilon^{-1 / 4} e^{i S(t) / \epsilon} e^{i p(t)(x-q(t)) / \epsilon}\left\{a^{0}\left(\frac{x-q(t)}{\sqrt{\epsilon}}, t\right) \chi_{n}\left(\frac{x}{\epsilon} ; p(t)\right)\right. \\
& \left.+\sqrt{\epsilon}\left[a^{1}\left(\frac{x-q(t)}{\sqrt{\epsilon}}, t\right) \chi_{n}\left(\frac{x}{\epsilon} ; p(t)\right)+\left(-i \partial_{y}\right) a^{0}\left(\frac{x-q(t)}{\sqrt{\epsilon}}, t\right) \partial_{p} \chi_{n}\left(\frac{x}{\epsilon} ; p(t)\right)\right]\right\} .
\end{aligned}
$$

Then, we have the following mild generalization of the result of Theorem 1.1 in 47):

Theorem 2.6 (Order 1 wave-packet with order $\sqrt{\epsilon}$ correction). Assume the same setting as in Theorem 2.4. in particular that the Isolated Band Property 2.2 holds along the trajectory $(p(t), q(t))$ of the classical Hamiltonian $\mathcal{H}_{n}=E_{n}(p)+W(q)$ for $t \in\left[t_{0}, t_{1}\right)$, where $t_{0}<t_{1} \leq \infty$.

Let $S(t)$ be as in 2.13) with initial action $S(0)=S_{0} \in \mathbb{R}$. Let $a^{0}(y, t)$ as in 2.14) and $a^{1}(y, t)$ be as in (2.21) with $a_{0}^{0}(y)$ and $a_{0}^{1}(y) \in \mathcal{S}(\mathbb{R})$.

Then, for sufficiently small $\epsilon>0$, we have that the unique solution $\psi^{\epsilon}(x, t)$ of the initial value problem (1.1) with approximate 'Bloch wavepacket' initial data with corrections proportional to $\sqrt{\epsilon}$ given at $t=t_{0}$ :

$$
\begin{aligned}
& i \epsilon \partial_{t} \psi^{\epsilon}=H^{\epsilon} \psi^{\epsilon} \\
& \psi^{\epsilon}\left(x, t_{0}\right)=\mathrm{WP}^{1, \epsilon}\left[S_{0}, q_{0}, p_{0}, a_{0}^{0}(y), a_{0}^{1}(y), \chi_{n}\left(z ; p_{0}\right)\right](x)+O_{L_{x}^{2}}(\epsilon)
\end{aligned}
$$


evolves as a modulated 'Bloch wavepacket' plus a corrector $\eta^{\epsilon}(x, t)$ :

$(2.24) \psi^{\epsilon}(x, t)=\mathrm{WP}^{1, \epsilon}\left[S(t), q(t), p(t), a^{0}(y, t), a^{1}(y, t), \chi_{n}(z ; p(t))\right](x, t)+\eta^{\epsilon}(x, t)$

where the corrector $\eta^{\epsilon}$ satisfies, for $t \in\left[t_{0}, t_{1}\right)$, the bound:

$$
\left\|\eta^{\epsilon}(\cdot, t)\right\|_{L^{2}} \leq C e^{c t} \epsilon
$$

where the constants $C>0, c>0$ are as stated in Theorem 2.4.

Furthermore, it follows that if $M\left(t_{0}, \infty\right)>0$, then we have the following error bound on the Ehrenfest time-scale:

$$
\sup _{t \in[0, \tilde{C} \ln 1 / \epsilon]}\left\|\eta^{\epsilon}(\cdot, t)\right\|_{L^{2}}=o(\sqrt{\epsilon}),
$$

where $\tilde{C}$ is any constant such that $\tilde{C}<\frac{1}{2 c}$, cf. 2.19.

Remark 2.7. By a natural extension of the methods of $[6]$ and $[47]$ one may derive, for any integer $k \geq 0$, 'kth-order Bloch wavepacket' approximate solutions:

$$
\mathrm{WP}^{k, \epsilon}\left[S(t), q(t), p(t), a^{0}(y, t), a^{1}(y, t), a^{2}(y, t), \ldots, a^{k}(y, t), \chi_{n}(z ; p(t))\right](x, t)
$$

such that the exact solution $\psi^{\epsilon}(x, t)$ of (1.1) with ' $k$-th order Bloch wavepacket' initial data:

$$
\psi_{0}^{\epsilon}(x)=\mathrm{WP}^{k, \epsilon}\left[S_{0}, q_{0}, p_{0}, a_{0}^{0}(y), a_{0}^{1}(y), a_{0}^{2}(y), \ldots, a_{0}^{k}(y), \chi_{n}\left(z ; p_{0}\right)\right](x)
$$

satisfies:

$$
\begin{aligned}
\psi^{\epsilon}(x, t)= & \mathrm{WP}^{k, \epsilon}\left[S(t), q(t), p(t), a^{0}(y, t), a^{1}(y, t), a^{2}(y, t), \ldots, a^{k}(y, t), \chi_{n}(z ; p(t))\right](x, t) \\
& +o_{L_{x}^{2}}\left(\epsilon^{k / 2}\right)
\end{aligned}
$$

up to 'Ehrenfest time' $t \sim \ln 1 / \epsilon$. Note that each function $\mathrm{WP}^{k, \epsilon}[\ldots](x, t)$ depends on $k+1$ envelope functions $a^{0}(y, t), a^{1}(y, t), a^{2}(y, t), \ldots, a^{k}(y, t)$, each of which satisfies a suitable Schrödinger equation driven by the $k$ previously defined envelope functions. Hence $a^{2}(y, t)$ satisfies a Schrödinger equation driven by $a^{0}(y, t)$ and $a^{1}(y, t)$ and so on.

\section{Statement of Results on Dynamics at BAnd CROSSings}

3.1. Linear band crossings. We next give a precise discussion of the character of one-dimensional Bloch band degeneracies (band crossings). The following property describes a linear band crossing, illustrated in Figures 2 and 4 . In Theorem 3.2 we assert that all band crossings in one dimension are of this type:

Property 3.1 (Linear band crossing). Let $E_{n}(p), E_{n+1}(p)$ denote two spectral band functions satisfying (2.3) for $p \in \mathcal{B}$. Let $p^{*} \in U$, where $U$ is an open subset of $\mathcal{B}$, be such that:

(A1) The bands $E_{n}$ and $E_{n+1}$ are degenerate at $p^{*}$, and this degeneracy is unique in $U$ :

$$
\begin{aligned}
& E_{n}\left(p^{*}\right)=E_{n+1}\left(p^{*}\right) \\
& \text { if } \tilde{p}^{*} \in U \text { and } E_{n}\left(\tilde{p}^{*}\right)=E_{n+1}\left(\tilde{p}^{*}\right) \text {, then } \tilde{p}^{*}=p^{*} .
\end{aligned}
$$

(A2) The bands $E_{n+1}, E_{n}$ are uniformly isolated from the rest of the spectrum for all $p \in U$, i.e. there exists a positive constant $M>0$ such that:

$$
\min _{p \in \bar{U}} \min _{m \notin\{n, n+1\}}\left\{\left|E_{m}(p)-E_{n+1}(p)\right|,\left|E_{n}(p)-E_{m}(p)\right|\right\} \geq M>0
$$


(A3) The maps:

$$
\begin{aligned}
& p \mapsto\left(E_{+}(p), \chi_{+}(z ; p)\right):= \begin{cases}\left(E_{n}(p), \chi_{n}(z ; p)\right) & \text { for } p \in U \text { and } p<p^{*} \\
\left(E_{n+1}(p), \chi_{n+1}(z ; p)\right) & \text { for } p \in U \text { and } p \geq p^{*}\end{cases} \\
& p \mapsto\left(E_{-}(p), \chi_{-}(z ; p)\right):= \begin{cases}\left(E_{n+1}(p), \chi_{n+1}(z ; p)\right) & \text { for } p \in U \text { and } p<p^{*} \\
\left(E_{n}(p), \chi_{n}(z ; p)\right) & \text { for } p \in U \text { and } p \geq p^{*}\end{cases}
\end{aligned}
$$

are smooth for all $p \in U$.

(A4) The bands $E_{+}, E_{-}$satisfy $\partial_{p} E_{+}\left(p^{*}\right)>0, \partial_{p} E_{-}\left(p^{*}\right)<0$ and in particular:

$$
\partial_{p} E_{+}\left(p^{*}\right)-\partial_{p} E_{-}\left(p^{*}\right)=2 \partial_{p} E_{+}\left(p^{*}\right)>0 .
$$

Caveat Lector! In (3.3), the notation + and - refers to the slope of the smooth band functions at the crossing point: $\partial_{p} E_{+}\left(p^{*}\right)>0, \partial_{p} E_{-}\left(p^{*}\right)<0$. This is not to be confused with an ordering of the bands themselves. Indeed, with our conventions we have:

$$
\text { for } p \in U \text { and } p<p^{*}: E_{+}(p)=E_{n}(p)<E_{n+1}(p)=E_{-}(p) \text {. }
$$

It is useful to view the functions $E_{+}(p), E_{-}(p)$ as smooth continuations of the band functions $E_{n}(p), E_{n+1}(p)$ from the interval $\left\{p \in U: p \leq p^{*}\right\}$ to the interval $\left\{p \in U: p>p^{*}\right\}$. We will refer to any band crossing satisfying Property 3.1 as a linear crossing. In one spatial dimension, all band crossings are linear. Moreover, crossings can only occur at 0 or $\pi$ (modulo $2 \pi$ ):

Theorem 3.2. Let $E_{n}(p), E_{n+1}(p)$ denote spectral band functions satisfying (2.3) for $p \in \mathcal{B}$, and let $p^{*} \in \mathcal{B}$ be such that $E_{n}\left(p^{*}\right)=E_{n+1}\left(p^{*}\right)$. Then:

(1) $p^{*}=0$ or $\pi$ (modulo $2 \pi$ ).

(2) There exists an open interval $U$ containing $p^{*}$ such that hypotheses (A1)(A4) of Property 3.1 hold.

The proof of Theorem 3.2 follows from the fact that the eigenvalue problem 2.2 has the form of an ordinary differential equation with real and periodic coefficients and may therefore be put into the form of Hill's equation, for which a well-developed theory exists 36, 11, 40. For a self-contained proof, see Appendix B.1 of [46].

Corollary 3.3. Let $E_{n}(p), E_{n+1}(p)$ denote spectral band functions in one dimension which cross at some $p^{*} \in \mathcal{B}$. Let $P_{ \pm}^{\perp}(p)$ denote the projection onto the orthogonal complement in $L_{p e r}^{2}$ of the functions $\chi_{+}(z ; p), \chi_{-}(z ; p)$, defined for $p \in U$ by (3.3). Then:

$$
\left\|\left(H(p)-E_{\sigma}(p)\right)^{-1} P_{ \pm}^{\perp}(p)\right\|_{L_{p e r}^{2} \rightarrow H_{p e r}^{2}} \leq \frac{1}{M}, \quad \sigma= \pm, p \in \bar{U} .
$$

where $M>0$ is the constant appearing in (3.2).

The bound (3.6) follows immediately from $(3.2)$. When we consider the dynamics of wavepackets associated with $E_{n}(p)$ or $E_{n+1}(p)$ and spectrally localized close to $p^{*}$, the gap condition (3.2) and Corollary 3.3 will allow us to bound contributions to the solution from all bands other than $E_{n}(p)$ and $E_{n+1}(p)$ uniformly through the crossing time, see Appendix D of [47] for details.

Remark 3.4. Theorem 3.2 does not generalize to spatial dimensions larger than one. Indeed, at so-called 'conical' or 'Dirac' points, which occur in the spectral band structure of two-dimensional periodic Schrödinger operators with honeycomb 
lattice symmetry, the local band structure is the union of Lipschitz surfaces $[16,17]$ and the map $p \mapsto \chi_{n}(z ; p)$ from the Brillouin zone to the Bloch eigenfunctions is discontinuous $[17]$.

\subsection{Examples of potentials with linear band crossings.}

Example 3.5 ( $m$ - gap potentials). Let $\omega_{1}, \omega_{3} \in \mathbb{C}$ with $\operatorname{Im}\left(\omega_{3} / \omega_{1}\right) \neq 0$. Define $\wp \omega_{1}, \omega_{3}(z)$, the Weierstrass elliptic function with periods $2 \omega_{1}, 2 \omega_{3}$ by:

$$
\wp \omega_{1}, \omega_{3}(z):=\frac{1}{z^{2}}+\sum_{\substack{(m, n) \in \mathbb{Z} \times \mathbb{Z},(m, n) \neq(0,0)}} \frac{1}{\left(z-2 m \omega_{1}-2 n \omega_{3}\right)^{2}}-\frac{1}{\left(2 m \omega_{1}+2 n \omega_{3}\right)^{2}} .
$$

The function $\wp_{\omega_{1}, \omega_{3}}(z)$ is doubly-periodic and even:

$$
\begin{aligned}
& \wp \omega_{1}, \omega_{3}\left(z+2 \omega_{1}\right)=\wp \omega_{1}, \omega_{3}\left(z+2 \omega_{3}\right)=\wp \omega_{1}, \omega_{3}(z) \\
& \wp \omega_{1}, \omega_{3}(-z)=\wp \omega_{1}, \omega_{3}(z),
\end{aligned}
$$

and has poles of degree two at the points $\Omega_{m, n}=2 m \omega_{1}+2 n \omega_{3}$ for all $(m, n) \in \mathbb{Z} \times \mathbb{Z}$. If $\omega_{1}=\omega, \omega_{3}=i \omega^{\prime}$ with $\omega, \omega^{\prime} \in \mathbb{R}$ and $\omega>0$, then $\wp_{\omega, i \omega^{\prime}}(z)$ is real for $z$ such that $\operatorname{Re} z \in\{0, \omega\}$ or $\operatorname{Im} z \in\left\{0, \omega^{\prime}\right\}$ by the symmetries [3.8). Now fix $\omega=1 / 2$, and define for any $\omega^{\prime} \in \mathbb{R}$ with $\omega^{\prime} \neq 0$ and positive integer $m$ :

$$
V(z):=\frac{m(m+1)}{2} \wp_{1 / 2, i \omega^{\prime}}\left(z+i \omega^{\prime}\right)
$$

Then for $z \in \mathbb{R}, V(z)$ is a real, smooth, 1-periodic function.

The $m$ lowest Bloch band dispersion functions defined by 2.3 for this potential are non-degenerate for all $p \in \mathcal{B}$, but for every $n>m$, the band $E_{n}(p)$ has a linear crossing with the band $E_{n+1}(p)$ at $p=0$ or $\left.p=\pi \sqrt{36}\right]$. Such potentials are known as ' $m$-gap' potentials since the $L^{2}(\mathbb{R})$ spectrum of the operator $-\frac{1}{2} \partial_{z}^{2}+V(z)$ in this case consists of $m+1$ real intervals with $m$ 'gaps' between them. Indeed, all ' $m$ gap' potentials, for positive integers $m$, are elliptic functions [29]. Any Weierstrass elliptic function may be written in terms of Jacobi elliptic functions; for more detail see [5, 48, 9, 1, 38].

The lowest three bands of a 'one-gap' potential are shown in Figure 2. The smooth bands at the linear crossing between the second and third bands defined by (3.3), whose existence is ensured by Theorem 3.2, are shown in Figure 4.

Example 3.6 (Trivial band crossings). Every Bloch band of a $1 / 2$ - periodic function regarded as a 1-periodic function is degenerate. To see this, let $V(z)$ be 1/2periodic. We may plot the band structure of the operator $-\frac{1}{2} \partial_{z}^{2}+V(z)$ with respect to the natural $4 \pi$-periodic Brillouin zone, which we take for concreteness to be $[0,4 \pi]$. We may also treat $V(z)$ as a 1-periodic potential and plot its band structure with respect to the $2 \pi$-periodic Brillouin zone $[0,2 \pi]$. But it is clear that any eigenpair of the 1/2-periodic eigenvalue problem will also be an eigenpair of the 1-periodic eigenvalue problem. Hence the band structure of the 1-periodic operator is nothing but the band structure of the 1/2-periodic operator 'folded over' onto the shorter interval. More precisely, eigenvalues of the 1/2-periodic operator with quasi-momentum $p \in[2 \pi, 4 \pi]$ will be eigenvalues of the 1-periodic operator with quasi-momentum $p-2 \pi$. For an example, see Figure 5. The converse of this argument also holds: whenever all 'odd-numbered' gaps close, then $V(z)$ is 1/2-periodic. Analogous statements hold for $1 / 2^{n}$-periodic potentials for any natural number $n[4,30,40]$. We will refer to such crossings as 'trivial', since they may be removed by a proper choice 


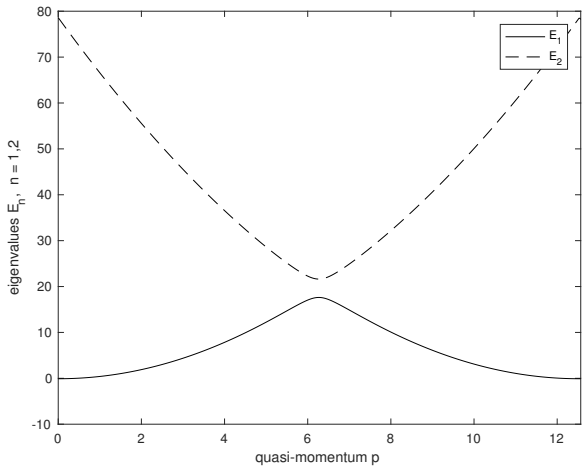

(A)

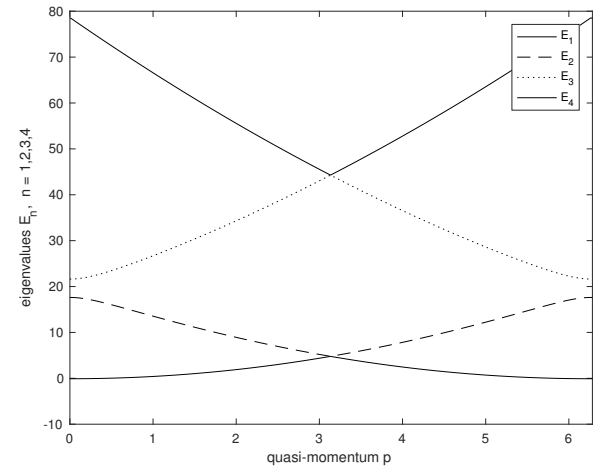

(B)

Figure 5. Lowest Bloch bands when $V(z)=4 \cos (4 \pi z)$, viewed as a $1 / 2$-periodic potential and plotted over the natural Brillouin zone in this case $[0,4 \pi](\mathrm{A})$ and viewed as a 1-periodic potential and plotted over $[0,2 \pi](\mathrm{B})$. When $V(z)$ is viewed as a 1-periodic potential, every Bloch band is degenerate at $p=\pi$.

of Brillouin zone. The amplitude of the 'excited' wave due to such crossings is zero; see Remark 3.14 and Appendix B.

3.3. Band crossing dynamics. We now make precise the scenario of a wavepacket whose quasi-momentum is driven by the external potential $W$ towards a quasimomentum $p^{*} \in \mathcal{B}$ at which there is a linear band crossing; see Property 3.1 .

Property 3.7 (Band Crossing Scenario). Let $E_{n}, E_{n+1}$ denote spectral band functions associated with the eigenvalue problem 2.3) for $p \in \mathcal{B}$ which have a linear crossing in the sense of Property 3.1 at $p^{*}$. Let $q_{0}, p_{0} \in \mathbb{R} \times \mathcal{B}$ be such that $G\left(E_{n}\left(p_{0}\right)\right)>0$ (i.e. the band $E_{n}(p)$ is isolated at $p_{0}$ : recall the definition of the spectral gap function $G(2.8)$ ). We assume the existence of a positive constant $t^{*}>0$ such that the equations of motion of the classical Hamiltonian $\mathcal{H}_{n}(q, p):=E_{n}(p)+W(q)$ :

$$
\begin{array}{ll}
\dot{q}(t)=\partial_{p} E_{n}(p(t)) & \dot{p}(t)=-\partial_{q} W(q(t)) \\
q(0)=q_{0} & p(0)=p_{0}
\end{array}
$$

have a unique smooth solution $(q(t), p(t)) \subset \mathbb{R} \times \mathcal{B}$ for all $t \in\left[0, t^{*}\right)$ such that the Bloch band function $E_{n}$ is isolated when evaluated at $p(t)$ for every $t \in\left[0, t^{*}\right)$ :

$$
\text { for all } t \in\left[0, t^{*}\right), G\left(E_{n}(p(t))\right)>0 \text { and } \lim _{t \uparrow t^{*}} p(t)=p^{*} \text {. }
$$

Let $q^{*}$ denote the limit: $\lim _{t \uparrow t^{*}} q(t)$. We assume that the wavepacket is 'driven' towards the crossing in the following sense:

$$
\lim _{t \uparrow t^{*}} \dot{p}(t)=-\partial_{q} W\left(q^{*}\right)>0 .
$$

Remark 3.8. We choose the sign of $-\partial_{q} W\left(q^{*}\right)$ in 3.12 to be positive without loss of generality. Note that it follows from (3.12) that for $t<t^{*}$ with $\left|t-t^{*}\right|$ sufficiently small, $p(t)<p^{*}$ : i.e. the wave-packet quasi-momentum approaches $p^{*}$ 'from the 
left'. As a consequence the 'smooth extension' of the map $t \mapsto E_{n}(p(t))$ for $t \geq t^{*}$ makes use of $E_{+}(p(t))$ rather than $E_{-}(p(t))$; see Proposition 3.9.

We aim to describe the solution of the PDE (1.1) with 'Bloch wavepacket' initial data of the form:

$$
\psi^{\epsilon}(x, 0)=\mathrm{WP}^{1, \epsilon}\left[S_{0}, q_{0}, p_{0}, a_{0}^{0}(y), a_{0}^{1}(y), \chi_{n}\left(z ; p_{0}\right)\right](x) ;
$$

in the Band Crossing Scenario (Property 3.7) up to errors of $o_{L^{2}}(\sqrt{\epsilon})$ for $t$ up to and greater than the crossing time $t^{*}$. Here, $a_{0}^{0}, a_{0}^{1} \in \mathcal{S}(\mathbb{R}), S_{0} \in \mathbb{R}$, and the $\mathrm{WP}^{1, \epsilon}[\ldots]$ notation is as in 2.22 .

Note that for $t<t^{*}$, 3.11) implies that Property 2.2 holds with $t_{0}=0$ and $t_{1}=t$. By Theorem 2.6 the solution $\psi^{\epsilon}(x, t)$ of (1.1) satisfies, for fixed $t$ and $\epsilon \downarrow 0$ :

$$
\psi^{\epsilon}(x, t)=\mathrm{WP}^{1, \epsilon}\left[S(t), q(t), p(t), a^{0}(y, t), a^{1}(y, t), \chi_{n}(z ; p(t))\right](x, t)+O_{L^{2}}(\epsilon)
$$

where $q(t), p(t), S(t), a^{0}(y, t), a^{1}(y, t)$ are as in 3.10 , 2.13, 2.14), and 2.21) respectively.

Two difficulties arise in estimating the error term in (3.14) for $t \geq t^{*}$ :

Difficulty 1. The functions $q(t), p(t), S(t), a^{0}(y, t), a^{1}(y, t), \chi_{n}(z ; p(t))$, and $\partial_{p} \chi_{n}(z ; p(t))$, and therefore the function:

$$
\mathrm{WP}^{1, \epsilon}\left[S(t), q(t), p(t), a^{0}(y, t), a^{1}(y, t), \chi_{n}(z ; p(t))\right](x, t),
$$

are not well-defined at $t=t^{*}$ since the band function $E_{n}(p)$ and its associated eigenfunctions $\chi_{n}(z ; p)$ are not smooth in $p$ at $p^{*}$.

Difficulty 2. The $L_{x}^{2}$-norm of the error in the approximation (3.14 depends directly on the inverse of the spectral gap function $G\left(E_{n}(p(t))\right)$, which blows up as $t \uparrow t^{*}$ since $\left|G\left(E_{n}(p(t))\right)\right| \sim\left|E_{n+1}(p(t))-E_{n}(p(t))\right| \downarrow 0$.

We return to Difficulty 2 below: see Theorem 3.12 and Corollary 3.16

3.4. Resolution of Difficulty 1; Smooth Continuation of Bands. Difficulty 1 may be overcome by making proper use of the smooth band functions $E_{+}, E_{-}$; see 3.3 in Property 3.1. Theorem 3.2 and Figure 4. The following proposition shows how in the Band Crossing Scenario (Property 3.7), we may extend the map $\left[0, t^{*}\right) \rightarrow \mathbb{R} \times \mathcal{B}, t \mapsto(q(t), p(t))$ to a smooth map over an interval $[0, T]$ with $T>t^{*}$ using the smooth band function $E_{+}$:

Proposition 3.9. Assume the Band Crossing Scenario (Property 3.7) with crossing occurring for $t=t^{*}$. Then for sufficiently small positive $\delta$ with $0<\delta<t^{*}$, the equations of motion of the classical Hamiltonian $\mathcal{H}_{+}(q, p):=E_{+}(p)+W(q)$ with data specified at $t^{*}$ :

$$
\begin{array}{ll}
\dot{q}_{+}(t)=\partial_{p} E_{+}\left(p_{+}(t)\right), & \dot{p}_{+}(t)=-\partial_{q} W\left(q_{+}(t)\right) \\
q_{+}\left(t^{*}\right)=q^{*} & p_{+}\left(t^{*}\right)=p^{*}
\end{array}
$$

have a unique smooth solution $\left(q_{+}(t), p_{+}(t)\right) \subset \mathbb{R} \times U$ over the interval $t \in\left[t^{*}-\right.$ $\left.\delta, t^{*}+\delta\right]$ which satisfies:

$$
\text { for all } t \in\left[t^{*}-\delta, t^{*}\right), q(t)=q_{+}(t), p(t)=p_{+}(t) .
$$


Furthermore, for sufficiently small $T \geq t^{*}+\delta>0$, there exists a solution $\left(q_{n+1}(t), p_{n+1}(t)\right)$ $\subset \mathbb{R} \times \mathcal{B}$ of the equations of motion of the classical Hamiltonian $\mathcal{H}_{n+1}(q, p):=$ $E_{n+1}(p)+W(q)$ over the interval $t \in\left(t^{*}, T\right]$ satisfying the limits:

$$
\begin{array}{ll}
\dot{q}_{n+1}(t)=\partial_{p} E_{n+1}\left(p_{n+1}(t)\right) & \dot{p}_{n+1}(t)=-\partial_{q} W\left(q_{n+1}(t)\right) \\
\lim _{t \downarrow t^{*}} q_{n+1}(t)=q^{*} & \lim _{t \downarrow t^{*}} p_{n+1}(t)=p^{*}
\end{array}
$$

such that $G\left(E_{n+1}\left(p_{n+1}(t)\right)\right)>0$ for all $t \in\left(t^{*}, T\right]$. This solution satisfies:

$$
\text { for all } t \in\left(t^{*}, t^{*}+\delta\right], q_{+}(t)=q_{n+1}(t), p_{+}(t)=p_{n+1}(t) .
$$

It follows from (3.17) and (3.19) that the map:

$$
t \mapsto\left(\mathfrak{q}_{+}(t), \mathfrak{p}_{+}(t)\right):= \begin{cases}(q(t), p(t)) & \text { for } t \in\left[0, t^{*}-\delta\right] \\ \left(q_{+}(t), p_{+}(t)\right) & \text { for } t \in\left[t^{*}-\delta, t^{*}+\delta\right] \\ \left(q_{n+1}(t), p_{n+1}(t)\right) & \text { for } t \in\left[t^{*}+\delta, T\right]\end{cases}
$$

is smooth as a map $[0, T] \rightarrow \mathbb{R} \times \mathcal{B}$.

Proof. The potential $W$ is smooth by assumption, the band functions $E_{n}, E_{n+1}$ are smooth everywhere away from $p^{*}$, and the band function $E_{+}$is smooth in $U$, a neighborhood of $p^{*}$. The proposition then follows easily from existence and uniqueness for solutions of ODEs with smooth coefficients.

Corresponding to the smooth extension $t \mapsto\left(\mathfrak{q}_{+}(t), \mathfrak{p}_{+}(t)\right)$ we may define the smooth extension of $\chi_{n}(z ; p(t))$ through the crossing:

$$
t \mapsto \mathfrak{X}_{+}\left(z ; \mathfrak{p}_{+}(t)\right):= \begin{cases}\chi_{n}(z ; p(t)) & \text { for } t \in\left[0, t^{*}-\delta\right] \\ \chi_{+}\left(z ; p_{+}(t)\right) & \text { for } t \in\left[t^{*}-\delta, t^{*}+\delta\right] . \\ \chi_{n+1}\left(z ; p_{n+1}(t)\right) & \text { for } t \in\left[t^{*}+\delta, T\right]\end{cases}
$$

Finally, using the smooth maps $t \mapsto\left(\mathfrak{q}_{+}(t), \mathfrak{p}_{+}(t)\right)$ and $t \mapsto \mathfrak{X}_{+}\left(z ; \mathfrak{p}_{+}(t)\right)$, we introduce smooth extensions of the functions $a^{0}(y, t), a^{1}(y, t)$, and $S(t)$ over the whole interval $t \in[0, T]$ as follows:

Definition 3.10 (Smooth extensions of $a^{0}(y, t), a^{1}(y, t)$, and $\left.S(t)\right)$. Let:

$$
S^{*}:=\lim _{t \uparrow t^{*}} S(t), a^{0, *}(y):=\lim _{t \uparrow t^{*}} a^{0}(y, t), \text { and } a^{1, *}(y):=\lim _{t \uparrow t^{*}} a^{1}(y, t) .
$$

Then let $S_{+}(t), a_{+}^{0}(y, t)$, and $a_{+}^{1}(y, t)$ be defined for $t \in\left[t^{*}-\delta, t^{*}+\delta\right]$ by 2.13), (2.14), and (2.21) with $t_{0}=t^{*}$, and where all dependence on $p(t), q(t), E_{n}(p(t))$, $\chi_{n}(z ; p(t))$, and $W(q(t))$ is replaced by dependence on $p_{+}(t), q_{+}(t), E_{+}\left(p_{+}(t)\right)$, $\chi_{+}\left(z ; p_{+}(t)\right)$, and $W\left(q_{+}(t)\right)$ respectively, and:

$$
S_{+}\left(t^{*}\right)=S^{*}, a_{+}^{0}\left(y, t^{*}\right)=a^{0, *}(y) \text {, and } a_{+}^{1}\left(y, t^{*}\right)=a^{1, *}(y) .
$$

Then let $S_{n+1}(t), a_{n+1}^{0}(y, t), a_{n+1}^{1}(y, t)$ be defined for $t \in\left(t^{*}, T\right]$ by equations (2.13), (2.14), and 2.21), replacing dependence on $p(t), q(t), E_{n}(p(t)), \chi_{n}(z ; p(t))$, and $W(q(t))$ by dependence on $p_{n+1}(t), q_{n+1}(t), E_{n+1}\left(p_{n+1}(t)\right), \chi_{n+1}\left(z ; p_{n+1}(t)\right)$, and $W\left(q_{n+1}(t)\right)$ and the limits:

$$
\lim _{t \downarrow t^{*}} S_{n+1}(t)=S^{*}, \lim _{t \downarrow t^{*}} a_{n+1}(y, t)=a^{0, *}(y), \text { and } \lim _{t \downarrow t^{*}} a^{1}(y, t)=a^{1, *}(y) .
$$


We denote by $\mathfrak{S}_{+}(t), \mathfrak{a}_{+}^{0}(y, t)$, and $\mathfrak{a}_{+}^{1}(y, t)$ smooth maps defined over the whole interval $t \in[0, T]$ defined analogously to 3.20 so that, for example:

$$
t \mapsto \mathfrak{a}_{+}^{0}(y, t):= \begin{cases}a^{0}(y, t) & \text { for } t \in\left[0, t^{*}-\delta\right] \\ a_{+}^{0}(y, t) & \text { for } t \in\left[t^{*}-\delta, t^{*}+\delta\right] . \\ a_{n+1}^{0}(y, t) & \text { for } t \in\left[t^{*}+\delta, T\right]\end{cases}
$$

We now define a first-order wavepacket smoothly continued through the crossing, an expression which is smooth for all $t \in[0, T]$ by (recall the definition of $\mathrm{WP}^{1, \epsilon}$ (2.22):

$$
\begin{aligned}
& \mathrm{WP}^{1, \epsilon}\left[\mathfrak{S}_{+}(t), \mathfrak{q}_{+}(t), \mathfrak{p}_{+}(t), \mathfrak{a}_{+}^{0}(y, t), \mathfrak{a}_{+}^{1}(y, t), \mathfrak{X}_{+}\left(z ; \mathfrak{p}_{+}(t)\right)\right](x, t):= \\
& := \begin{cases}\mathrm{WP}^{1, \epsilon}\left[S(t), q(t), p(t), a^{0}(y, t), a^{1}(y, t), \chi_{n}(z ; p(t))\right](x, t) & \text { for } t \in\left[0, t^{*}-\delta\right] \\
\mathrm{WP}^{1, \epsilon}\left[S_{+}(t), q_{+}(t), p_{+}(t), a_{+}^{0}(y, t), a_{+}^{1}(y, t), \chi_{+}(z ; p(t))\right](x, t) & \text { for } t \in\left[t^{*}-\delta, t^{*}+\delta\right] . \\
\mathrm{WP}^{1, \epsilon}\left[S_{n+1}(t), q_{n+1}(t), p_{n+1}(t), a_{n+1}^{0}(y, t), a_{n+1}^{1}(y, t), \chi_{n+1}(z ; p(t))\right](x, t) & \text { for } t \in\left[t^{*}+\delta, T\right]\end{cases}
\end{aligned}
$$

Remark 3.11. By construction, (3.26) is a wavepacket associated with the band $E_{n}$ for $t \in\left[0, t^{*}-\delta\right]$, a wavepacket associated with the band $E_{n+1}$ for $t \in\left[t^{*}+\delta, T\right]$, and a wavepacket associated with the 'smooth transition' $E_{+}$for $t \in\left[t^{*}-\delta, t^{*}+\delta\right]$.

3.5. Resolution of Difficulty 2 Incorporation of the second band and a new, fast / non-adiabatic, time-scale. We first present a result which quantifies, through a blow-up rate of the error bound, the breakdown of the single band approximation (3.14) as $t \uparrow t^{*}$ :

Theorem 3.12. Assume the Band Crossing Scenario (Property 3.7). Assume $a_{0}^{0}(y)$ and $a_{0}^{1}(y) \in \mathcal{S}(\mathbb{R})$ and let $\psi^{\epsilon}(x, t)$ denote the unique solution of (1.1) with 'Bloch wavepacket' initial data:

$$
\psi^{\epsilon}(x, 0)=\mathrm{WP}_{n}^{1, \epsilon}\left[S_{0}, q_{0}, p_{0}, a_{0}^{0}(y), a_{0}^{1}(y), \chi_{n}\left(z ; p_{0}\right)\right](x) .
$$

Then for $t \in\left[0, t^{*}\right), \psi^{\epsilon}(x, t)$ satisfies:

$$
\psi^{\epsilon}(x, t)=\mathrm{WP}^{1, \epsilon}\left[\mathfrak{S}_{+}(t), \mathfrak{q}_{+}(t), \mathfrak{p}_{+}(t), \mathfrak{a}_{+}^{0}(y, t), \mathfrak{a}_{+}^{1}(y, t), \mathfrak{X}_{+}\left(z ; \mathfrak{p}_{+}(t)\right)\right](x)+\eta^{\epsilon}(x, t)
$$

where $\mathrm{WP}_{+}^{1, \epsilon}(x, t)$ is given by (3.26). Moreover, the corrector $\eta^{\epsilon}(x, t)$ satisfies the following bound for $0<t<t^{*}$, which blows up as $t \uparrow t^{*}$ :

$$
\begin{aligned}
\left\|\eta^{\epsilon}(\cdot, t)\right\|_{L^{2}} \leq & C\left|\left\langle\chi_{-}\left(\cdot ; p^{*}\right) \mid \partial_{p} \chi_{+}\left(\cdot ; p^{*}\right)\right\rangle\right|\left(\frac{\epsilon}{\left|t-t^{*}\right|}+\frac{\epsilon^{3 / 2}}{\left|t-t^{*}\right|^{2}}\right) \\
& +O\left(\epsilon, \epsilon^{3 / 2} \ln \left|t-t^{*}\right|, \frac{\epsilon^{3 / 2}}{\left|t-t^{*}\right|}\right)
\end{aligned}
$$

The constants in (3.29) (explicit and implied) are independent of $t, \epsilon$ and are finite as long as: $\partial_{p} E_{+}\left(p^{*}\right)-\partial_{p} E_{-}\left(p^{*}\right)=2 \partial_{p} E_{+}\left(p^{*}\right)>0$ and $\partial_{q} W\left(q^{*}\right) \neq 0$.

The proof of Theorem 3.12 is by explicit term by term estimation of the corrector $\eta^{\epsilon}(x, t)$. We demonstrate this procedure in Section 4 for the term which contributes the first term on the right-hand side of the estimate (3.29). This term dominates when $t=t^{*}-\epsilon^{\xi}$ where $0<\xi<1 / 2$ and hence controls the time interval of validity of the single-band ansatz (see Corollary 3.16). All other terms in $\eta^{\epsilon}$ may be bounded similarly, see Appendix B.3 of [46]. 
Remark 3.13. The manner in which the nonzero constants $\partial_{p} E_{+}\left(p^{*}\right)-\partial_{p} E_{-}\left(p^{*}\right)=$ $2 \partial_{p} E_{+}\left(p^{*}\right)$ and $\partial_{q} W\left(q^{*}\right)$ play a role in the bound 3.29 is seen in 4.22.

Theorem 3.12 shows that the single band ansatz, even when smoothly continued through the linear band crossing, fails to give a good approximation (error of size $\left.o_{L_{x}^{2}}(\sqrt{\epsilon})\right)$ to the solution $\psi^{\epsilon}(x, t)$ of equation (1.1) for small $\left|t-t^{*}\right|$. Furthermore, since the dominant terms in the bound $\left(3.29, \sqrt{\epsilon} \times\left(\sqrt{\epsilon} /\left|t-t^{*}\right|\right), \sqrt{\epsilon} \times\left(\sqrt{\epsilon} /\left|t-t^{*}\right|\right)^{2}\right.$, are proportional to

$$
\left\langle\chi_{-}\left(\cdot ; p^{*}\right) \mid \partial_{p} \chi_{+}\left(\cdot ; p^{*}\right)\right\rangle,
$$

we see that the failure of the single band wave-packet approximation is due to contributions to the solution from the other band participating in the linear crossing, $p \mapsto E_{-}(p)$, growing to be of size $\sim \sqrt{\epsilon}$ when:

$$
\left|t-t^{*}\right| \sim \sqrt{\epsilon}
$$

Remark 3.14. At 'trivial' crossings, which occur when the potential $V(z)$ has minimal period 1/2 (recall Example 3.6), the 'inter-band coupling coefficient' (3.30) is zero (see Appendix B). It follows that the amplitude of the wave associated with the other band involved in the crossing 'excited' (Theorem 3.20) at the crossing is also zero. This is consistent with the observation that the crossing may be removed by making the proper choice of Brillouin zone.

Remark 3.15. When $V(z)$ is an $m$-gap potential (recall Example 3.5), the eigenfunctions $\chi_{ \pm}(z ; p)$ may be explicitly displayed (see [5], for example), and the 'coupling coefficient' (3.30) may be numerically computed relatively easily. Such a computation for the lowest band crossing of the 'one-gap' potential (shown in Figures 2 and (4) shows that (3.30) is non-zero in this case.

The following Corollary of Theorem 3.12 precisely characterizes the time interval of validity of the single band ansatz:

Corollary 3.16. Let $t=t^{*}-\epsilon^{\xi}$. Then, for small enough $\epsilon>0$, (3.29) implies that the corrector function $\eta^{\epsilon}(x, t)$ which appears in (3.28) satisfies:

$$
\sup _{t \in\left[0, t^{*}-\epsilon^{\xi}\right]}\left\|\eta^{\epsilon}(\cdot, t)\right\|_{L^{2}} \leq C \epsilon^{1-\xi}
$$

where $C>0$ is a constant independent of $\epsilon, \xi, t$. In particular, if $0<\xi<1 / 2$, then:

$$
\sup _{t \in\left[0, t^{*}-\epsilon^{\xi}\right]}\left\|\eta^{\epsilon}(\cdot, t)\right\|_{L^{2}}=o(\sqrt{\epsilon}) .
$$

It follows that $\eta^{\epsilon}(x, t)$ is negligible in $L^{2}(\mathbb{R})$ compared with $\mathrm{WP}^{1, \epsilon}$ in the expansion (3.28) for $t \in\left[0, t^{*}-\epsilon^{\xi}\right]$.

In order to describe the solution for $t \sim t^{*}$ and $t \geq t^{*}$, it is necessary to make a more general ansatz for the solution which accounts for the excitation of a wave associated with the other band involved in the crossing over the time-scale:

$$
s:=\frac{t-t^{*}}{\sqrt{\epsilon}}=\mathcal{O}(1) .
$$

The following proposition, which is analogous to Proposition 3.9, is required to construct this excited wave: 
Proposition 3.17. Assume the Band Crossing Scenario (Property 3.7). Then for sufficiently small positive $\delta^{\prime}$ the equations of motion of the classical Hamiltonian $\mathcal{H}_{-}(q, p):=E_{-}(p)+W(q)$ with data specified at $t^{*}$ :

$$
\begin{array}{ll}
\dot{q}_{-}(t)=\partial_{p} E_{-}\left(p_{-}(t)\right) & \dot{p}_{-}(t)=-\partial_{q} W\left(q_{-}(t)\right) \\
q_{-}\left(t^{*}\right)=q^{*} & p_{-}\left(t^{*}\right)=p^{*}
\end{array}
$$

have a unique smooth solution $\left(q_{-}(t), p_{-}(t)\right) \subset \mathbb{R} \times U$ over the interval $t \in\left[t^{*}-\right.$ $\left.\delta^{\prime}, t^{*}+\delta^{\prime}\right]$. Furthermore, for sufficiently small $T^{\prime} \geq t^{*}+\delta^{\prime}>0$, there exists a solution $\left(q_{n}(t), p_{n}(t)\right) \subset \mathbb{R} \times \mathcal{B}$ of the equations of motion of the classical Hamiltonian $\mathcal{H}_{n}(q, p):=E_{n}(p)+W(q)$ over the interval $t \in\left(t^{*}, T^{\prime}\right]$ satisfying the limits:

$$
\begin{array}{ll}
\dot{q}_{n}(t)=\partial_{p} E_{n}\left(p_{n}(t)\right) & \dot{p}_{n}(t)=-\partial_{q} W\left(q_{n}(t)\right) \\
\lim _{t \downarrow t^{*}} q_{n}(t)=q^{*} & \lim _{t \downarrow t^{*}} p_{n}(t)=p^{*}
\end{array}
$$

such that $G\left(E_{n}\left(p_{n}(t)\right)\right)>0$ for all $t \in\left(t^{*}, T^{\prime}\right]$. This solution satisfies:

$$
\text { for all } t \in\left(t^{*}, t^{*}+\delta^{\prime}\right], q_{-}(t)=q_{n}(t), p_{-}(t)=p_{n}(t) .
$$

It follows from 3.38 that the map:

$$
\left(\mathfrak{q}_{-}(t), \mathfrak{p}_{-}(t)\right):= \begin{cases}\left(q_{-}(t), p_{-}(t)\right) & \text { for } t \in\left[t^{*}-\delta^{\prime}, t^{*}+\delta^{\prime}\right] \\ \left(q_{n}(t), p_{n}(t)\right) & \text { for } t \in\left[t^{*}+\delta^{\prime}, T\right] .\end{cases}
$$

is smooth over the interval $t \in\left[t^{*}-\delta^{\prime}, T^{\prime}\right]$.

We again define, as in 3.21 :

$$
t \mapsto \mathfrak{X}_{-}\left(z ; \mathfrak{p}_{-}(t)\right):=\left\{\begin{array}{ll}
\chi_{-}\left(z ; p_{-}(t)\right) & \text { for } t \in\left[t^{*}-\delta^{\prime}, t^{*}+\delta^{\prime}\right] \\
\chi_{n}\left(z ; p_{n}(t)\right) & \text { for } t \in\left[t^{*}+\delta^{\prime}, T^{\prime}\right]
\end{array} .\right.
$$

The precise form of the wave 'excited' at the crossing time is derived from a rigorous multiscale analysis on the emergent nonadiabatic time-scale (3.34) (see Section 5.1). The following definition is the result of this calculation:

Definition 3.18 (Parameters of the excited wave-packet). We let $S_{-}(t)$ and $a_{-}^{0}(y, t)$ be defined for $t \in\left[t^{*}-\delta^{\prime}, t^{*}+\delta^{\prime}\right]$ by (2.13) and (2.14) with $t_{0}=t^{*}$, in which all dependence on $p(t), q(t), E_{n}(p(t)), \chi_{n}(z ; p(t))$, and $W(q(t))$ replaced by dependence on $p_{-}(t), q_{-}(t), E_{-}\left(p_{-}(t)\right), \chi_{-}\left(z ; p_{-}(t)\right)$, and $W\left(q_{-}(t)\right)$ respectively.

Moreover, $S_{-}\left(t^{*}\right)=S^{*}$ and the initial data for $a_{-}^{0}(y, t)$, generated by the incoming ' + band' wave-packet is given by:

$$
\begin{aligned}
& a_{-}^{0}\left(y, t^{*}\right)=\partial_{q} W\left(q^{*}\right) \times\left\langle\chi_{-}\left(\cdot ; p^{*}\right) \mid \partial_{p} \chi_{+}\left(\cdot ; p^{*}\right)\right\rangle \\
& \quad \times \int_{-\infty}^{\infty} e^{i\left[\partial_{q} W\left(q^{*}\right)\right]\left[\partial_{p} E_{+}\left(p^{*}\right)-\partial_{p} E_{-}\left(p^{*}\right)\right] \tau^{2} / 2} \times a^{0, *}\left(y-\left[\partial_{p} E_{+}\left(p^{*}\right)-\partial_{p} E_{-}\left(p^{*}\right)\right] \tau\right) \mathrm{d} \tau .
\end{aligned}
$$

Here, $S^{*}=\lim _{t \uparrow t^{*}} S(t)$ and $a^{0, *}(y)=\lim _{t \uparrow t^{*}} a^{0}(y, t)(3.22)$.

Recall that $\partial_{q} W\left(q^{*}\right)$ is assumed to be non-zero (see (3.12)) and that $\partial_{p} E_{+}\left(p^{*}\right)-$ $\partial_{p} E_{-}\left(p^{*}\right)=2 \partial_{p} E_{+}\left(p^{*}\right)$ is always nonzero at band crossings (Theorem 3.2. Property $3.1(A 4))$ and hence the integral in (3.41) is well-defined since $a^{0, *}(y)$ is localized. We then define $S_{n}(t), a_{n}^{0}(y, t)$ for $t \in\left(t^{*}, T^{\prime}\right]$ by replacing dependence on $p(t)$, 
$q(t), E_{n}(p(t)), \chi_{n}(z ; p(t))$, and $W(q(t))$ by dependence on $p_{n}(t), q_{n}(t), E_{n}\left(p_{n}(t)\right)$, $\chi_{n}\left(z ; p_{n}(t)\right)$, and $W\left(q_{n}(t)\right)$ respectively, and by the limits:

$$
\lim _{t \downarrow t^{*}} S_{n}(t)=S^{*}, \lim _{t \downarrow t^{*}} a_{n}^{0}(y, t)=a^{0, *}(y) .
$$

We denote by $\mathfrak{S}_{-}(t), \mathfrak{a}_{-}^{0}(y, t)$ the smooth maps defined over the whole interval $t \in\left[t^{*}-\delta^{\prime}, T^{\prime}\right]$ in analogy with the definitions of $\mathfrak{S}_{+}(t)$ and $\mathfrak{a}_{+}(y, t)$ in (3.25).

Remark 3.19. Equation (3.41) gives the precise form of the envelope of the wavepacket 'excited' at the crossing time $t^{*}$. In Appendix A we prove using this expression that the main statement (3.45) of Theorem 3.20 (below) is consistent with the solution of an appropriate simplified 'Landau-Zener' model.

We now define the wave-packet associated with the band $E_{-}$which is 'excited' at the crossing time $t^{*}$ by:

$$
\begin{aligned}
& \mathrm{WP}^{0, \epsilon}\left[\mathfrak{S}_{-}(t), \mathfrak{q}_{-}(t), \mathfrak{p}_{-}(t), \mathfrak{a}_{-}^{0}(y, t), \mathfrak{X}_{-}\left(z ; \mathfrak{p}_{-}(t)\right)\right](x, t):= \\
& := \begin{cases}\mathrm{WP}^{0, \epsilon}\left[S_{-}(t), q_{-}(t), p_{-}(t), a_{-}^{0}(y, t), \chi_{-}(z ; p(t))\right](x, t) & \text { for } t \in\left[t^{*}-\delta^{\prime}, t^{*}+\delta^{\prime}\right] \\
\mathrm{WP}^{0, \epsilon}\left[S_{n}(t), q_{n}(t), p_{n}(t), a_{n}^{0}(y, t), \chi_{n}(z ; p(t))\right](x, t) & \text { for } t \in\left[t^{*}+\delta^{\prime}, T\right]\end{cases}
\end{aligned}
$$

3.6. The main theorem. Our main theorem is that a size 1 incoming wave-packet associated with the ' + band', when encountering a band-crossing, generates a size 1 'transmitted + band' wave-packet and a 'reflected - band' wave-packet of size $\sqrt{\epsilon}$. Moreover, when the wave-packet is in a neighborhood of the crossing, i.e. $t \approx t_{*}$ and hence $(p(t), q(t)) \approx\left(p_{*}, q_{*}\right)$, the detailed dynamics are non-adiabatic and are described by an ansatz incorporating wave-packets from both bands with envelopes varying on an additional fast scale. The precise statement is the following:

Theorem 3.20. Assume the Band Crossing Scenario (Property 3.7) in which the crossing time, along the trajectory $(p(t), q(t))$ is $t=t_{*}$. Let $\xi$, $\xi^{\prime}$ be fixed such that $3 / 8<\xi^{\prime}<\xi<1 / 2$. Let $\tilde{T}>0$, with $0<t_{*}<\tilde{T}$, be sufficiently small that Propositions 3.9 and 3.17 hold with $T=\tilde{T}$ and $T^{\prime}=\tilde{T}$ respectively.

Let $\psi^{\epsilon}(x, t)$ denote the unique solution of (1.1) with 'incident Bloch wavepacket' initial data (3.2\%), defined for $t \in[0, \tilde{T}]$.

Then, there exists an $\epsilon_{0}>0$ such that for all $0<\epsilon<\epsilon_{0}$ the following holds.

(1) For $t \in\left[0, t^{*}-\epsilon^{\xi}\right), \psi^{\epsilon}(x, t)$ may be approximated up to errors of $o_{L_{x}^{2}}(\sqrt{\epsilon})$ by a single-band ansatz (see Theorem 3.12 and Corollary 3.16):

$$
\psi^{\epsilon}(x, t)=\mathrm{WP}^{1, \epsilon}\left[\mathfrak{S}_{+}(t), \mathfrak{q}_{+}(t), \mathfrak{p}_{+}(t), \mathfrak{a}_{+}^{0}(y, t), \mathfrak{a}_{+}^{1}(y, t), \mathfrak{X}_{+}\left(z ; \mathfrak{p}_{+}(t)\right)\right](x, t)+o_{L_{x}^{2}}(\sqrt{\epsilon}) .
$$

(2) For $t \in\left(t^{*}+\epsilon^{\xi}, \tilde{T}\right], \psi^{\epsilon}(x, t)$ is approximated up to errors of $o_{L_{x}^{2}}(\sqrt{\epsilon})$ by the sum of two Bloch wave-packets, one associated with each band involved in the crossing (recall Definition 3.18):

$$
\begin{aligned}
& \psi^{\epsilon}(x, t)=\mathrm{WP}^{1, \epsilon}\left[\mathfrak{S}_{+}(t), \mathfrak{q}_{+}(t), \mathfrak{p}_{+}(t), \mathfrak{a}_{+}^{0}(y, t), \mathfrak{a}_{+}^{1}(y, t), \mathfrak{X}_{+}\left(z ; \mathfrak{p}_{+}(t)\right)\right](x, t) \\
& +\sqrt{\epsilon} \mathrm{WP}^{0, \epsilon}\left[\mathfrak{S}_{-}(t), \mathfrak{q}_{-}(t), \mathfrak{p}_{-}(t), \mathfrak{a}_{-}^{0}(y, t), \mathfrak{X}_{-}\left(z ; \mathfrak{p}_{-}(t)\right)\right](x, t)+o_{L_{x}^{2}}(\sqrt{\epsilon}) .
\end{aligned}
$$

Over the interval $t \in\left(t^{*}-\epsilon^{\xi^{\prime}}, t^{*}+\epsilon^{\xi^{\prime}}\right)$, the solution $\psi^{\epsilon}(x, t)$ is expressible, with errors of size $o_{L_{x}^{2}}(\sqrt{\epsilon})$, by a superposition of wave-packets from both + and - bands, 
whose amplitudes vary on an additional (fast / non-adiabatic) time scale:

$$
s:=\frac{t-t^{*}}{\sqrt{\epsilon}} .
$$

The detailed construction appears in Section 5.1 .

Remark 3.21. The restriction to sufficiently small $\tilde{T}>0$ in Theorem 3.20 is to ensure that neither the incident nor excited wavepacket encounter a second band crossing over the time interval $t \in[0, \tilde{T}]$. It is clear that this assumption may be relaxed and the analysis repeated each time a wavepacket is incident on a band crossing in order to obtain results valid over arbitrary finite time intervals, fixed independent of $\epsilon$.

Remark 3.22. By construction:

$$
\mathrm{WP}^{1, \epsilon}\left[\mathfrak{S}_{+}(t), \mathfrak{q}_{+}(t), \mathfrak{p}_{+}(t), \mathfrak{a}_{+}^{0}(y, t), \mathfrak{a}_{+}^{1}(y, t), \mathfrak{X}_{+}\left(z ; \mathfrak{p}_{+}(t)\right)\right](x, t)
$$

is a wavepacket with $L_{x}^{2}$-norm proportional to 1 associated with the band $E_{n}$ for $t \in\left[0, t^{*}-\delta\right]$ and with the band $E_{n+1}$ for $t \in\left[t^{*}+\delta, \tilde{T}\right]$, and:

$$
\sqrt{\epsilon} \mathrm{WP}^{0, \epsilon}\left[\mathfrak{S}_{-}(t), \mathfrak{q}_{-}(t), \mathfrak{p}_{-}(t), \mathfrak{a}_{-}^{0}(y, t), \mathfrak{X}_{-}\left(z ; \mathfrak{p}_{-}(t)\right)\right](x, t)
$$

is a wavepacket with $L_{x}^{2}$-norm proportional to $\sqrt{\epsilon}$ associated with the band $E_{n}$ for $t \in$ $\left[t^{*}+\delta^{\prime}, \tilde{T}\right]$. Hence the statement of Theorem 3.20 is consistent with the description of our results given in Section 1 .

Remark 3.23. To leading order in $\sqrt{\epsilon}$, the center of mass of the wavepacket 'excited' at the crossing is given by $\mathfrak{q}_{-}(t)$, which, for $\left|t-t^{*}\right|$ small enough, evolves according to (3.35). The center of mass of the incoming wavepacket is given by (again to leading order in $\sqrt{\epsilon}) \mathfrak{q}_{+}(t)$, which evolves (again for $\left|t-t^{*}\right|$ small enough) according to (3.16). Since $\dot{\mathfrak{q}}_{+}\left(t^{*}\right)=\partial_{p} E_{+}\left(p^{*}\right)>0$ and $\dot{\mathfrak{q}}_{-}\left(t^{*}\right)=\partial_{p} E_{-}\left(p^{*}\right)<0$ we have that the velocities of the centers of mass of each wavepacket have opposite signs: see Figure 3 .

Remark 3.24. The $\mathcal{O}(\sqrt{\epsilon})$ reflected wave is required only to describe the solution with an order of size $o(\sqrt{\epsilon})$. Indeed, by dropping terms of o(1) in $L_{x}^{2}$ in (3.44), (3.45, and in the asymptotic solution which we construct for $t \in\left(t^{*}-\epsilon^{\xi^{\prime}}, t^{*}+\epsilon^{\xi^{\prime}}\right)$, we have that, under the assumptions of Theorem 3.20, for all $t \in[0, \tilde{T}]$ :

$$
\psi^{\epsilon}(x, t)=\mathrm{WP}^{0, \epsilon}\left[\mathfrak{S}_{+}(t), \mathfrak{q}_{+}(t), \mathfrak{p}_{+}(t), \mathfrak{a}_{+}^{0}(y, t), \mathfrak{X}_{+}\left(z ; \mathfrak{p}_{+}(t)\right)\right](x, t)+o_{L_{x}^{2}}(1) .
$$

Equation (3.49) may be summarized as follows: to leading order in $\sqrt{\epsilon}$, the wavepacket propagates as if associated with an isolated band, with band function given by the smooth extension of $E_{n}$ through the crossing. This is consistent with previous results obtained by Hagedorn [27] and Jecko et al. [31, 10] on dynamics at codimension 1 eigenvalue band crossings.

\section{Sketch of Proof of Theorem 3.12 on Blow-Up of ERror in SINGLE-BAND APPROXIMATION AS $t$ APPROACHES THE CROSSING TIME $t^{*}$}

4.1. Strategy for estimating the corrector. In this section we recall the simple Lemma which we use in the proofs of Theorem 3.12 and Theorem 3.20 to estimate the corrector to a wave-packet approximate solution. A similar strategy was followed in 6, 47 
Lemma 4.1. For $0<T \leq \infty$, let $\psi^{\epsilon} \in C^{0}\left(\left[t_{0}, T\right) ; L^{2}(\mathbb{R})\right)$ denote the unique solution of the initial value problem (1.1) with initial data $\psi_{0}^{\epsilon}(x)$ given at $t=t_{0}$ :

$$
\begin{aligned}
& i \epsilon \partial_{t} \psi^{\epsilon}=H^{\epsilon} \psi^{\epsilon} \\
& \psi^{\epsilon}\left(x, t_{0}\right)=\psi_{0}^{\epsilon}(x) .
\end{aligned}
$$

Furthermore, let $\psi_{a p p}^{\epsilon}(x, t) \in C^{0}\left(\left[t_{0}, T\right) ; L^{2}(\mathbb{R})\right), r^{\epsilon}(x, t)$ be such that:

$$
\begin{aligned}
& i \epsilon \partial_{t} \psi_{a p p}^{\epsilon}=H^{\epsilon} \psi_{a p p}^{\epsilon}+r^{\epsilon} \\
& \psi_{a p p}^{\epsilon}\left(x, t_{0}\right)=\psi_{a p p, 0}^{\epsilon}(x) .
\end{aligned}
$$

Introduce $\eta^{\epsilon}(x, t)$ defined by:

$$
\eta^{\epsilon}(x, t):=\psi^{\epsilon}(x, t)-\psi_{a p p}^{\epsilon}(x, t) .
$$

Then,

$$
\left\|\eta^{\epsilon}(\cdot, t)\right\|_{L^{2}} \leq\left\|\psi_{0}^{\epsilon}(\cdot)-\psi_{a p p, 0}^{\epsilon}(\cdot)\right\|_{L^{2}}+\frac{1}{\epsilon} \int_{t_{0}}^{t}\left\|r^{\epsilon}\left(\cdot, t^{\prime}\right)\right\|_{L^{2}} d t^{\prime} .
$$

Remark 4.2. We shall apply Lemma 4.1 with $\psi_{a p p}^{\epsilon}(x, t)$ equal to an approximate solution of 1.1 and $r^{\epsilon}(x, t)=\left(i \partial_{t}-H^{\epsilon}\right) \psi_{\text {app }}^{\epsilon}$, a sufficiently high order in $\sqrt{\epsilon}$ residual.

Proof. The function $\eta^{\epsilon}(x, t)$ satisfies the initial value problem:

$$
\begin{aligned}
& i \epsilon \partial_{t} \eta^{\epsilon}=H^{\epsilon} \eta^{\epsilon}+r^{\epsilon} \\
& \psi_{a p p}^{\epsilon}\left(x, t_{0}\right)=\psi_{0}^{\epsilon}(x)-\psi_{a p p, 0}^{\epsilon}(x)
\end{aligned}
$$

Multiplying both sides of $(4.5)$ by $\overline{\eta^{\epsilon}}$, taking the imaginary part and using selfadjointness of $H^{\epsilon}$ yields: $\epsilon \partial_{t}\left\|\eta^{\epsilon}\right\|_{L^{2}}^{2}=-i\left\langle\eta^{\epsilon} \mid r^{\epsilon}\right\rangle_{L^{2}}+i\left\langle r^{\epsilon} \mid \eta^{\epsilon}\right\rangle_{L^{2}}$. This implies, using the Cauchy-Schwarz inequality, that $2 \epsilon\left\|\eta^{\epsilon}\right\|_{L^{2}} \partial_{t}\left\|\eta^{\epsilon}\right\|_{L^{2}} \leq 2\left\|r^{\epsilon}\right\|_{L^{2}}\left\|\eta^{\epsilon}\right\|_{L^{2}}$. Cancelling common factors from both sides (note that the inequality is trivially true if $\left\|\eta^{\epsilon}\right\|_{L^{2}}=0$ ) and integrating from $t_{0}$ to $t$ gives (4.4).

We now estimate the error in the single-band approximation as $t \uparrow t^{*}$, as measured by the $L_{x}^{2}$-norm of the corrector function $\eta^{\epsilon}(x, t)$ which appears in 3.28$)$. We start by recalling the strategy of the proof of Theorem 2.6 the proof of Theorem 2.4 is similar. Let $\psi^{\epsilon}(x, t)$ denote the exact solution of (1.1) with approximate 'Bloch wavepacket' initial data (2.23) specified at $t=t_{0}$. Then by Lemma 4.1, if we can find an approximate solution of 1.1), $\psi_{\text {app }}^{\epsilon}(x, t)$, such that 4.2 holds with:

$$
\begin{gathered}
\left\|\psi_{0}^{\epsilon}(\cdot)-\left.\psi_{a p p}^{\epsilon}(\cdot, t)\right|_{t=0}\right\|_{L^{2}} \leq C \epsilon \\
\text { and }\left\|r^{\epsilon}(\cdot, t)\right\|_{L^{2}} \leq C e^{c t} \epsilon^{2},
\end{gathered}
$$

where the constants $C>0, c>0$ are independent of $\epsilon, t$, then it follows from 4.3 and 4.4 that:

$$
\left\|\psi^{\epsilon}(\cdot, t)-\psi_{a p p}^{\epsilon}(\cdot, t)\right\|_{L^{2}} \leq C \epsilon e^{c t} .
$$

If in addition we have that:

$$
\left\|\psi_{a p p}^{\epsilon}(\cdot, t)-\mathrm{WP}^{1, \epsilon}\left[S(t), q(t), p(t), a^{0}(y, t), a^{1}(y, t), \chi_{n}(z ; p(t))\right](\cdot, t)\right\|_{L^{2}} \leq C e^{c t} \epsilon,
$$

where $q(t), p(t)$ and so on are as in the statement of Theorem 2.6, then the conclusions of Theorem 2.6 follow immediately by the triangle inequality. The details of how to construct such a $\psi_{a p p}^{\epsilon}(x, t)$ were presented in 47 . 
Theorem 2.6 implies, in particular, that the solution $\psi^{\epsilon}(x, t)$ of 1.1 with initial data (3.27) satisfies:

$$
\begin{aligned}
& \text { for } t \in\left[0, t^{*}-\delta\right] \\
& \left\|\psi^{\epsilon}(\cdot, t)-\mathrm{WP}^{1, \epsilon}\left[S(t), q(t), p(t), a^{0}(y, t), a^{1}(y, t), \chi_{n}(z ; p(t))\right](\cdot, t)\right\|_{L^{2}}=O_{L_{x}^{2}}(\epsilon) .
\end{aligned}
$$

Due to the band crossing at $p^{*}$, the Isolated Band Property 2.2 does not hold as $t \uparrow t^{*}$. As a result the proof of Theorem 2.6 fails as follows:

(1) As $t \uparrow t^{*}$, the $L_{x}^{2}$-norm of the residual $r^{\epsilon}(x, t)$ defined by 4.2 , diverges.

(2) The integral $\frac{1}{\epsilon} \int_{0}^{t}\left\|r^{\epsilon}\left(\cdot, t^{\prime}\right)\right\|_{L^{2}} \mathrm{~d} t^{\prime}$, and hence the bound 4.4) on the $L^{2}$-norm of the corrector function $\eta^{\epsilon}(x, t)$ diverges as $t \uparrow t^{*}$.

Theorem 3.12 is proved by analyzing the rates of blow-up of singular terms in $r^{\epsilon}(x, t)$ and then deducing the resulting rate of blow-up of the bound (4.4). In Section 4.2 we explain the strategy by studying the term which contributes the first term on the right-hand side of the estimate 3.29 , which is the dominant term when $t=t^{*}-\epsilon^{\xi}$ with $0<\xi<1 / 2$ and controls the time interval of validity of the single-band ansatz (Corollary 3.16). For a sketch of the general argument, see Appendix B.3 of 46 .

4.2. Estimation of representative term demonstrating blow-up as $t \uparrow t^{*}$. Let $t \in\left[t^{*}-\delta, t^{*}\right]$ where $\delta>0$ is as in Proposition 3.9 so that:

$$
\begin{aligned}
& \mathrm{WP}^{1, \epsilon}\left[\mathfrak{S}_{+}(t), \mathfrak{q}_{+}(t), \mathfrak{p}_{+}(t), \mathfrak{a}_{+}^{0}(y, t), \mathfrak{a}_{+}^{1}(y, t), \mathfrak{X}_{+}\left(z ; \mathfrak{p}_{+}(t)\right)\right](x, t) \\
& =\mathrm{WP}^{1, \epsilon}\left[S_{+}(t), q_{+}(t), p_{+}(t), a_{+}^{0}(y, t), a_{+}^{1}(y, t), \chi_{+}(z ; p(t))\right](x, t)
\end{aligned}
$$

Here, $q_{+}(t), p_{+}(t)$ are as in $(3.16), S_{+}(t), a_{+}^{0}(y, t), a_{+}^{1}(y, t)$ are as in Definition 3.10 and $E_{+}(p), \chi_{+}(z ; p)$ are as in (3.3). The representative term which appears in the residual $r^{\epsilon}(x, t) 4.2$ which we now consider is the following:

$$
\begin{aligned}
& R^{\epsilon}(x, t):=\epsilon^{-1 / 4} e^{i \phi_{+}^{\epsilon}(y, t) / \epsilon}[ \\
& \left.\epsilon^{2}\left(-i \partial_{t}\right)\left(-i \partial_{q} W\left(q_{+}(t)\right) a_{+}^{0}(y, t) \mathcal{R}_{+}\left(p_{+}(t)\right) P_{+}^{\perp}\left(p_{+}(t)\right) \partial_{p} \chi_{+}\left(z ; p_{+}(t)\right)\right)\right]\left.\right|_{y=\frac{x-q_{+}(t)}{\epsilon^{1 / 2}, z=\frac{x}{\epsilon}}}
\end{aligned}
$$

where $\phi_{+}^{\epsilon}(y, t):=S_{+}(t)+\epsilon^{1 / 2} p_{+}(t) y$

Here, $P_{+}^{\perp}(p)$ denotes the projection operator onto the orthogonal complement of the subspace of $L_{p e r}^{2}$ spanned by $\chi_{+}(z ; p)$, and:

$$
\mathcal{R}_{+}(p):=\left(H(p)-E_{+}(p)\right)^{-1}
$$

denotes the resolvent operator where $H(p)$ is as in 2.3). Because of the band crossing at $p^{*}$, the operator $\mathcal{R}_{+}(p) P_{+}^{\perp}(p)$ is singular as $p \rightarrow p^{*}$ on the 'resonant' subspace of $L_{p e r}^{2}$ spanned by $\chi_{-}(z ; p)$. The operator $\mathcal{R}_{+}(p) P_{ \pm}^{\perp}(p)$ however, where $P_{ \pm}^{\perp}(p)$ is defined as the projection onto the orthogonal complement of $\chi_{+}(z ; p)$ and $\chi_{-}(z ; p)$ in $L_{p e r}^{2}$ is regular for all $p \in U$ by (A2) of Property 3.1 (see Corollary 3.3). 
We isolate the singular part of 4.12 as follows. Expressing $\partial_{p} \chi_{+}\left(z ; p_{+}(t)\right)$ in terms of its projections onto both $\chi_{+}\left(z ; p_{+}(t)\right)$ and $\chi_{-}\left(z ; p_{+}(t)\right)$, and their orthogonal complement, the range of $P_{ \pm}^{\perp}\left(p_{+}(t)\right)$, we have

$$
\begin{aligned}
& \mathcal{R}_{+}\left(p_{+}(t)\right) P_{+}^{\perp}\left(p_{+}(t)\right) \partial_{p} \chi_{+}\left(z ; p_{+}(t)\right)=\mathcal{R}_{+}(p(t)) P_{ \pm}^{\perp}\left(p_{+}(t)\right) \partial_{p} \chi_{+}\left(z ; p_{+}(t)\right) \\
& +\left(E_{-}\left(p_{+}(t)\right)-E_{+}\left(p_{+}(t)\right)\right)^{-1}\left\langle\chi_{-}\left(\cdot ; p_{+}(t)\right) \mid \partial_{p} \chi_{+}\left(\cdot ; p_{+}(t)\right)\right\rangle \chi_{-}(z ; p(t))
\end{aligned}
$$

Since $p_{+}(t) \rightarrow p^{*}$ as $t \uparrow t_{*}, E_{+}\left(p^{*}\right)=E_{-}\left(p^{*}\right)$, the singular behavior is isolated in the latter term of (4.14). We decompose $R^{\epsilon}(x, t)$ into its corresponding regular and singular parts:

$$
R^{\epsilon}(x, t):=R_{\text {regular }}^{\epsilon}(x, t)+R_{\text {singular }}^{\epsilon}(x, t)
$$

where:

$$
\begin{aligned}
& R_{\text {regular }}^{\epsilon}(x, t)=\epsilon^{-1 / 4} e^{i \phi_{+}^{\epsilon}(y, t) / \epsilon}[ \\
& \left.\epsilon^{2}\left(-i \partial_{t}\right)\left(-i \partial_{q} W\left(q_{+}(t)\right) a_{+}^{0}(y, t) \mathcal{R}_{+}\left(p_{+}(t)\right) P_{ \pm}^{\perp}\left(p_{+}(t)\right) \partial_{p} \chi_{+}\left(z ; p_{+}(t)\right)\right)\right]\left.\right|_{y=\frac{x-q_{+}(t)}{\epsilon^{1 / 2}}, z=\frac{x}{\epsilon}} \\
& R_{\text {singular }}^{\epsilon}(x, t)=\epsilon^{-1 / 4} e^{i \phi_{+}^{\epsilon}(y, t) / \epsilon}\left[\epsilon ^ { 2 } ( - i \partial _ { t } ) \left(-i \partial_{q} W\left(q_{+}(t)\right) a_{+}^{0}(y, t)\right.\right. \\
& \left.\left.\times\left(E_{-}\left(p_{+}(t)\right)-E_{+}\left(p_{+}(t)\right)\right)^{-1}\left\langle\chi_{-}\left(z ; p_{+}(t)\right) \mid \partial_{p} \chi_{+}\left(z ; p_{+}(t)\right)\right\rangle \chi_{-}\left(z ; p_{+}(t)\right)\right)\right]\left.\right|_{y=\frac{x-q_{+}(t)}{\epsilon^{1 / 2}, z=\frac{x}{\epsilon}}} .
\end{aligned}
$$

It follows from the techniques detailed in 47 that:

$$
R_{\text {regular }}^{\epsilon}(x, t)=O_{L_{x}^{2}}\left(\epsilon^{2}\right)
$$

uniformly as $t \uparrow t^{*}$. On the other hand, $R_{\text {singular }}^{\epsilon}(x, t)$ is explicitly singular, since it depends on $\left(E_{-}\left(p_{+}(t)\right)-E_{+}\left(p_{+}(t)\right)\right)^{-1}$ which is unbounded as $t \uparrow t^{*}$. The time derivative of $R_{\text {singular }}^{\epsilon}(x, t)$ yields two terms:

$$
R_{\text {singular }}^{\epsilon}(x, t)=R_{\text {singular }}^{1, \epsilon}(x, t)+R_{\text {singular }}^{2, \epsilon}(x, t),
$$

where:

$$
\begin{aligned}
& R_{\text {singular }}^{1, \epsilon}(x, t):=\epsilon^{-1 / 4} e^{i \phi_{+}^{\epsilon}(y, t) / \epsilon}\left[\epsilon^{2}\left(E_{-}\left(p_{+}(t)\right)-E_{+}\left(p_{+}(t)\right)\right)^{-1}\right. \\
& \left.\times\left(-i \partial_{t}\right)\left(-i \partial_{q} W\left(q_{+}(t)\right) a_{+}^{0}(y, t)\left\langle\chi_{-}\left(z ; p_{+}(t)\right) \mid \partial_{p} \chi_{+}\left(z ; p_{+}(t)\right)\right\rangle \chi_{-}\left(z ; p_{+}(t)\right)\right)\right]\left.\right|_{y=\frac{x-q_{+}(t)}{\epsilon^{1 / 2}}, z=\frac{x}{\epsilon}} \\
& R_{\text {singular }}^{2, \epsilon}(x, t):=\epsilon^{-1 / 4} e^{i \phi_{+}^{\epsilon}(y, t) / \epsilon}\left[\epsilon^{2} \partial_{t}\left(\left(E_{-}\left(p_{+}(t)\right)-E_{+}\left(p_{+}(t)\right)\right)^{-1}\right)\right. \\
& \left.\times(-i)\left(-i \partial_{q} W\left(q_{+}(t)\right) a_{+}^{0}(y, t)\left\langle\chi_{-}\left(z ; p_{+}(t)\right) \mid \partial_{p} \chi_{+}\left(z ; p_{+}(t)\right)\right\rangle \chi_{-}\left(z ; p_{+}(t)\right)\right)\right]\left.\right|_{y=\frac{x-q_{+}(t)}{\epsilon^{1 / 2}}, z=\frac{x}{\epsilon}} .
\end{aligned}
$$

We now concentrate on $R_{\text {singular }}^{2, \epsilon}(x, t)$ which will turn out to be the dominant term as $t \uparrow t^{*}$. We first evaluate the time-derivative:

$$
\begin{aligned}
& \partial_{t}\left(\left(E_{-}\left(p_{+}(t)\right)-E_{+}\left(p_{+}(t)\right)\right)^{-1}\right)= \\
& \partial_{q} W\left(q_{+}(t)\right)\left(\partial_{p} E_{-}\left(p_{+}(t)\right)-\partial_{p} E_{+}\left(p_{+}(t)\right)\right)\left(E_{-}\left(p_{+}(t)\right)-E_{+}\left(p_{+}(t)\right)\right)^{-2} .
\end{aligned}
$$


We then follow 6, 47. in estimating $R_{\text {singular }}^{2, \epsilon}$ in $L_{x}^{2}$ by taking the $L^{\infty}$ norm of all $z$-dependence and the $L^{2}$-norm of all $y$ dependence:

$$
\begin{aligned}
& \left\|R_{\text {singular }}^{2, \epsilon}(\cdot, t)\right\|_{L^{2}} \leq \epsilon^{2}\left|\partial_{q} W\left(q_{+}(t)\right)\right|^{2}\left|\partial_{p} E_{-}\left(p_{+}(t)\right)-\partial_{p} E_{+}\left(p_{+}(t)\right)\right| \\
& \times\left|E_{-}\left(p_{+}(t)\right)-E_{+}\left(p_{+}(t)\right)\right|^{-2}\left\|a_{+}^{0}(\cdot, t)\right\|_{L^{2}}\left|\left\langle\chi_{-}\left(\cdot ; p_{+}(t)\right) \mid \partial_{p} \chi_{+}\left(\cdot ; p_{+}(t)\right)\right\rangle_{L^{2}[0,1]}\right|\left\|\chi_{+}\left(\cdot, p_{+}(t)\right)\right\|_{L^{\infty}[0,1]} .
\end{aligned}
$$

Taylor-expanding in $t-t^{*}$, using the non-degeneracy conditions 3.4 and 3.12 , we have that as $t \uparrow t^{*}$ :

$$
\begin{aligned}
& \left|\left(E_{+}\left(p_{+}(t)\right)-E_{-}\left(p_{+}(t)\right)\right)^{-2}\right| \leq \\
& \left|\frac{1}{\partial_{q} W\left(q^{*}\right)\left(\partial_{p} E_{+}\left(p^{*}\right)-\partial_{p} E_{-}\left(p^{*}\right)\right)}\right|^{2}\left(\frac{1}{\left|t-t^{*}\right|^{2}}\right)+O\left(\frac{1}{\left|t-t^{*}\right|}\right) .
\end{aligned}
$$

Substituting 4.22 into 4.21 and Taylor-expanding all other terms gives:

$$
\begin{aligned}
& \left\|R_{\text {singular }}^{2, \epsilon}(\cdot, t)\right\|_{L^{2}} \leq \\
& \left|\frac{\left\langle\chi_{-}\left(\cdot ; p^{*}\right) \mid \partial_{p} \chi_{+}\left(\cdot ; p^{*}\right)\right\rangle_{L^{2}[0,1]}\left\|a_{+}^{0}\left(\cdot, t^{*}\right)\right\|_{L^{2}}\left\|\chi_{+}\left(\cdot, p^{*}\right)\right\|_{L^{\infty}[0,1]}}{\partial_{p} E_{-}\left(p^{*}\right)-\partial_{p} E_{+}\left(p^{*}\right)}\right|\left(\frac{\epsilon^{2}}{\left|t-t^{*}\right|^{2}}\right)+O\left(\frac{\epsilon^{2}}{\left|t-t^{*}\right|}\right)
\end{aligned}
$$

Similar analysis shows that:

$$
\left\|R_{\text {singular }}^{1, \epsilon}(\cdot, t)\right\|_{L^{2}}=O\left(\frac{\epsilon^{2}}{\left|t-t^{*}\right|}\right) .
$$

Recall the relationship between the residual $r^{\epsilon}(x, t)$ and the bound on the solution error $\eta^{\epsilon}(x, t):=\psi^{\epsilon}(x, t)-\psi_{a p p}^{\epsilon}(x, t)$ (4.4). Putting everything (4.15), 4.17), 4.23), and (4.24) together, then integrating once in time and dividing by $\epsilon$, we see that the term contributed by $R^{\epsilon}(x, t)$ to the solution error $\eta^{\epsilon}(x, t)$ may be bounded by:

$$
\begin{aligned}
& \frac{1}{\epsilon} \int_{0}^{t}\left\|R^{\epsilon}\left(\cdot, t^{\prime}\right)\right\|_{L^{2}} \mathrm{~d} t^{\prime} \leq \\
& \left|\frac{\left\langle\chi_{-}\left(\cdot ; p^{*}\right) \mid \partial_{p} \chi_{+}\left(\cdot ; p^{*}\right)\right\rangle_{L^{2}[0,1]}\left\|a_{+}^{0}\left(\cdot, t^{*}\right)\right\|_{L^{2}}\left\|\chi_{+}\left(\cdot, p^{*}\right)\right\|_{L^{\infty}[0,1]}}{\partial_{p} E_{-}\left(p^{*}\right)-\partial_{p} E_{+}\left(p^{*}\right)}\right|\left(\frac{\epsilon}{\left|t-t^{*}\right|}\right) \\
& +O\left(\epsilon, \epsilon \ln \left|t-t^{*}\right|\right) .
\end{aligned}
$$

It follows that the right hand side of (4.25), which is the bound on the corrector to the wave-packet ansatz, is of the same size as the $O\left(\epsilon^{1 / 2}\right)$ term in the wave-packet approximate solution for $\left|t-t_{*}\right| \sim \epsilon^{1 / 2}$.

\section{Proof of Theorem 3.20 on Coupled Band dynamics When $t \sim t^{*}$}

We now turn to the proof of Theorem 3.20 , on the dynamics through the crossing time $t^{*}$. We follow the strategy adopted by Hagedorn, see in particular the proof of Theorem 5.1 in 27 . Theorem 3.12 and Corollary 3.16 give a description of the exact solution $\psi^{\epsilon}(x, t)$ of 1.1 with initial data given by (3.27) which is valid with 
errors of $o_{L_{x}^{2}}\left(\epsilon^{1 / 2}\right)$ up to $t=t^{*}-\epsilon^{\xi}$ for any $\xi \in(0,1 / 2)$ :

For all $t \in\left[0, t^{*}-\epsilon^{\xi}\right)$,

$\psi^{\epsilon}(x, t)=\mathrm{WP}^{1, \epsilon}\left[\mathfrak{S}_{+}(t), \mathfrak{q}_{+}(t), \mathfrak{p}_{+}(t), \mathfrak{a}_{+}^{0}(y, t), \mathfrak{a}_{+}^{1}(y, t), \mathfrak{X}_{+}\left(z ; \mathfrak{p}_{+}(t)\right)\right](x, t)+o_{L_{x}^{2}}\left(\epsilon^{1 / 2}\right)$.

We seek to extend (5.1) to a description of $\psi^{\epsilon}(x, t)$ up to errors of $o_{L_{x}^{2}}\left(\epsilon^{1 / 2}\right)$ over the entire interval $t \in[0, \tilde{T}]$ where $\tilde{T}$ is chosen such that Propositions 3.9 and 3.17 hold with $T=\tilde{T}$ and $T^{\prime}=\tilde{T}$. We first claim that the proof of Theorem 3.20 may be reduced to (a) the construction of an 'inner solution' $\psi_{a p p, i n n e r}^{\epsilon}(x, t)$ satisfying certain properties and (b) an application of Lemma 4.1

Proposition 5.1. Let $\xi, \xi^{\prime} \in(0,1 / 2)$ be such that $\xi^{\prime}<\xi$ so that $\left(t^{*}-\epsilon^{\xi}, t^{*}+\right.$ $\left.\epsilon^{\xi}\right) \subset\left(t^{*}-\epsilon^{\xi^{\prime}}, t^{*}+\epsilon^{\xi^{\prime}}\right)$. Assume (5.1) for an incoming wave-packet. Consider an approximate solution $\psi_{\text {app,inner }}^{\epsilon}(x, t)$ which satisfies the following three properties:

(P1) $\psi_{a p p, i n n e r}^{\epsilon}(x, t)$ is equal to the single-band ansatz in the 'incoming' overlap region $t \in\left(t^{*}-\epsilon^{\xi^{\prime}}, t^{*}-\epsilon^{\xi}\right)$ up to errors of $o\left(\epsilon^{1 / 2}\right)$ in $L^{2}(\mathbb{R})$. That is,

for all $t \in\left(t^{*}-\epsilon^{\xi^{\prime}}, t^{*}-\epsilon^{\xi}\right)$,

$\left\|\psi_{\text {app }, \text { inner }}^{\epsilon}(\cdot, t)-\mathrm{WP}^{1, \epsilon}\left[\mathfrak{S}_{+}(t), \mathfrak{q}_{+}(t), \mathfrak{p}_{+}(t), \mathfrak{a}_{+}^{0}(y, t), \mathfrak{a}_{+}^{1}(y, t), \mathfrak{X}_{+}\left(z ; \mathfrak{p}_{+}(t)\right)\right](\cdot, t)\right\|_{L^{2}}=o\left(\epsilon^{1 / 2}\right)$,

(P2) $\psi_{\text {app,inner }}^{\epsilon}(x, t)$ is an approximate solution to 1.1$)\left(i \epsilon \partial_{t}-H^{\epsilon}\right) \psi^{\epsilon}=0$ :

$$
i \epsilon \partial_{t} \psi_{\text {app }, \text { inner }}^{\epsilon}-H^{\epsilon} \psi_{\text {app }, \text { inner }}^{\epsilon}=r_{\text {inner }}^{\epsilon}
$$

with residual satisfying the bound:

$$
\frac{1}{\epsilon} \int_{t^{*}-\epsilon^{\xi^{\prime}}}^{t^{*}+\epsilon^{\xi^{\prime}}}\left\|r_{\text {inner }}^{\epsilon}\left(\cdot, t^{\prime}\right)\right\|_{L^{2}} d t^{\prime}=o\left(\epsilon^{1 / 2}\right)
$$

(P3) $\psi_{\text {app,inner }}^{\epsilon}(x, t)$ matches the 'two-band' ansatz of (3.45) in the 'outgoing' overlap region $t \in\left(t^{*}+\epsilon^{\xi}, t^{*}+\epsilon^{\xi^{\prime}}\right)$ up to errors of $o\left(\epsilon^{1 / 2}\right)$ in $L^{2}(\mathbb{R})$. That is,

$$
\begin{aligned}
& \text { for all } t \in\left(t^{*}+\epsilon^{\xi}, t^{*}+\epsilon^{\xi^{\prime}}\right), \\
& \| \psi_{\text {app }, \text { inner }}(\cdot, t)-\mathrm{WP}^{1, \epsilon}\left[\mathfrak{S}_{+}(t), \mathfrak{q}_{+}(t), \mathfrak{p}_{+}(t), \mathfrak{a}_{+}^{0}(y, t), \mathfrak{a}_{+}^{1}(y, t), \mathfrak{X}_{+}\left(z ; \mathfrak{p}_{+}(t)\right)\right](\cdot, t) \\
& -\epsilon^{1 / 2} \mathrm{WP}^{0, \epsilon}\left[\mathfrak{S}_{-}(t), \mathfrak{q}_{-}(t), \mathfrak{p}_{-}(t), \mathfrak{a}_{-}^{0}(y, t), \mathfrak{X}_{-}\left(z ; \mathfrak{p}_{-}(t)\right)\right](\cdot, t) \|_{L^{2}}=o\left(\epsilon^{1 / 2}\right) .
\end{aligned}
$$

Then, under conditions (P1), (P2), and (P3), Theorem 3.20 holds.

Proof. We apply Lemma 4.1 with $t_{0}=t^{*}-\epsilon^{\xi^{\prime}}, t_{1}=t^{*}+\epsilon^{\xi^{\prime}}, \psi_{\text {app }}^{\epsilon}(x, t)=\psi_{\text {app,inner }}^{\epsilon}(x, t)$, and $r^{\epsilon}(x, t)=r_{a p p, \text { inner }}^{\epsilon}(x, t)$. It then follows from (P1) and (P2) that:

$$
\begin{aligned}
& \text { For all } t \in\left(t^{*}-\epsilon^{\xi^{\prime}}, t^{*}+\epsilon^{\xi^{\prime}}\right), \\
& \left\|\psi^{\epsilon}(\cdot, t)-\psi_{\text {app }, \text { inner }}^{\epsilon}(\cdot, t)\right\|_{L^{2}}=o\left(\epsilon^{1 / 2}\right) .
\end{aligned}
$$


Combining (5.6) with (P3), we have that:

$$
\begin{aligned}
& \text { For all } t \in\left(t^{*}+\epsilon^{\xi}, t^{*}+\epsilon^{\xi^{\prime}}\right), \\
& \| \psi^{\epsilon}(\cdot, t)-\mathrm{WP}^{1, \epsilon}\left[\mathfrak{S}_{+}(t), \mathfrak{q}_{+}(t), \mathfrak{p}_{+}(t), \mathfrak{a}_{+}^{0}(y, t), \mathfrak{a}_{+}^{1}(y, t), \mathfrak{X}_{+}\left(z ; \mathfrak{p}_{+}(t)\right)\right](\cdot, t) \\
& +\epsilon^{1 / 2} \mathrm{WP}^{0, \epsilon}\left[\mathfrak{S}_{-}(t), \mathfrak{q}_{-}(t), \mathfrak{p}_{-}(t), \mathfrak{a}_{-}^{0}(y, t), \mathfrak{X}_{-}\left(z ; \mathfrak{p}_{-}(t)\right)\right](x, t) \|_{L^{2}}=o\left(\epsilon^{1 / 2}\right) .
\end{aligned}
$$

We claim that the main statement (3.45) of Theorem 3.20 then follows from the isolated band theory. For any $\tilde{T}_{0}$ fixed independent of $\epsilon$ such that $t^{*}<\tilde{T}_{0}<\tilde{T}$, the Isolated Band Property 2.2 holds for the bands $E_{n}(p)$ and $E_{n+1}(p)$ and trajectories $p_{n}(t)$ and $p_{n+1}(t)$ defined by (3.37), (3.18) with $t_{0}=\tilde{T}_{0}, t_{1}=\tilde{T}$. By linearity, the two-band wavepacket ansatz agrees (modulo errors of $o_{L_{x}^{2}}\left(\epsilon^{1 / 2}\right)$ ) with the exact solution $\psi_{\text {outgoing }}^{\epsilon}(x, t)$ of the full equation $(1.1)$ over the interval $t \in\left[\tilde{T}_{0}, \tilde{T}\right]$ with initial data given at $\tilde{T}_{0}$ by:

$$
\begin{aligned}
& \psi_{\text {outgoing }}^{\epsilon}\left(x, \tilde{T}_{0}\right)= \\
& \operatorname{WP}^{1, \epsilon}\left[\mathfrak{S}_{+}\left(\tilde{T}_{0}\right), \mathfrak{q}_{+}\left(\tilde{T}_{0}\right), \mathfrak{p}_{+}\left(\tilde{T}_{0}\right), \mathfrak{a}_{+}^{0}\left(y, \tilde{T}_{0}\right), \mathfrak{a}_{+}^{1}\left(y, \tilde{T}_{0}\right), \mathfrak{X}_{+}\left(z ; \mathfrak{p}_{+}\left(\tilde{T}_{0}\right)\right)\right]\left(x, \tilde{T}_{0}\right) \\
& +\epsilon^{1 / 2} \mathrm{WP}^{0, \epsilon}\left[\mathfrak{S}_{-}\left(\tilde{T}_{0}\right), \mathfrak{q}_{-}\left(\tilde{T}_{0}\right), \mathfrak{p}_{-}\left(\tilde{T}_{0}\right), \mathfrak{a}_{-}^{0}\left(y, \tilde{T}_{0}\right), \mathfrak{X}_{-}\left(z ; \mathfrak{p}_{-}\left(\tilde{T}_{0}\right)\right)\right]\left(x, \tilde{T}_{0}\right) .
\end{aligned}
$$

By performing the same analysis as in the proof of Theorem 3.12 backwards in time towards $t^{*}$, we have that:

$$
\begin{aligned}
& \text { For all } t \in\left(t^{*}+\epsilon^{\xi}, \tilde{T}\right] \\
& \| \psi_{\text {outgoing }}^{\epsilon}(\cdot, t)-\mathrm{WP}^{1, \epsilon}\left[\mathfrak{S}_{+}(t), \mathfrak{q}_{+}(t), \mathfrak{p}_{+}(t), \mathfrak{a}_{+}^{0}(y, t), \mathfrak{a}_{+}^{1}(y, t), \mathfrak{X}_{+}\left(z ; \mathfrak{p}_{+}(t)\right)\right](\cdot, t) \\
& +\epsilon^{1 / 2} \mathrm{WP}^{0, \epsilon}\left[\mathfrak{S}_{-}(t), \mathfrak{q}_{-}(t), \mathfrak{p}_{-}(t), \mathfrak{a}_{-}^{0}(y, t), \mathfrak{X}_{-}\left(z ; \mathfrak{p}_{-}(t)\right)\right](x, t) \|_{L^{2}}=o\left(\epsilon^{1 / 2}\right) .
\end{aligned}
$$

But now combining the triangle inequality with (5.7), we have that:

$$
\begin{aligned}
& \text { For all } t \in\left(t^{*}+\epsilon^{\xi}, t^{*}+\epsilon^{\xi^{\prime}}\right), \\
& \left\|\psi^{\epsilon}(\cdot, t)-\psi_{\text {outgoing }}^{\epsilon}(\cdot, t)\right\|_{L^{2}}=o\left(\epsilon^{1 / 2}\right) .
\end{aligned}
$$

Since $\psi^{\epsilon}(x, t)$ and $\psi_{\text {outgoing }}^{\epsilon}(x, t)$ are both exact solutions of 1.1], applying Lemma 4.1 one more time with $\psi_{\text {app }}^{\epsilon}(x, t)=\psi_{\text {outgoing }}^{\epsilon}(x, t)$ gives that:

$$
\begin{aligned}
& \text { For all } t \in\left(t^{*}+\epsilon^{\xi}, \tilde{T}\right], \\
& \left\|\psi^{\epsilon}(\cdot, t)-\psi_{\text {outgoing }}^{\epsilon}(\cdot, t)\right\|_{L^{2}}=o\left(\epsilon^{1 / 2}\right) .
\end{aligned}
$$

The main statement of Theorem 3.20 3.45) then follows from combining (5.11) and (5.9).

This brings us to the core construction of the paper.

5.1. Derivation of $\psi_{a p p, i n n e r}^{\epsilon}$ satisfying hypotheses of Proposition 5.1. We make the following ansatz for $\psi_{a p p, i n n e r}^{\epsilon}(x, t)$, which incorporates both + and bands, and a new 'fast' timescale:

$$
s=\frac{t-t^{*}}{\epsilon^{1 / 2}}
$$


which was motivated by the preceding single-band analysis:

$(5.13)$

$\psi_{\text {app }, \text { inner }}^{\epsilon}(x, t)=\left.\epsilon^{-1 / 4} \sum_{\sigma= \pm} e^{i\left\{S_{\sigma}(t)+\epsilon^{1 / 2} p_{\sigma}(t) y_{\sigma}\right\} / \epsilon} f_{\sigma, \text { inner }}^{\epsilon}\left(y_{\sigma}, z, t, s\right)\right|_{y_{\sigma}=\frac{x-q_{\sigma}(t)}{\epsilon^{1 / 2}, z=\frac{x}{\epsilon}, s=\frac{t-t^{*}}{\epsilon^{1 / 2}}}}$.

The new time scale has been introduced in the envelope functions $f_{\sigma, \text { inner }}^{\epsilon}$. We take $\left(q_{\sigma}(t), p_{\sigma}(t)\right), \sigma= \pm$ as in (3.16 and 3.35), $S_{\sigma}(t), \sigma= \pm$, as in Definitions 3.10 and 3.18 and assume that $f_{\sigma, \text { inner }}^{\epsilon}\left(y_{\sigma}, z, t, s\right)$ may be expanded in powers of $\epsilon^{1 / 2}$ :

$$
f_{\sigma, i n n e r}^{\epsilon}\left(y_{\sigma}, z, t, s\right)=f_{\sigma, \text { inner }}^{0}\left(y_{\sigma}, z, t, s\right)+\epsilon^{1 / 2} f_{\sigma, \text { inner }}^{1}\left(y_{\sigma}, z, t, s\right)+\ldots
$$

Then, $\psi_{\text {app,inner }}^{\epsilon}$, given by (5.13), satisfies the non-homogeneous Schroedinger equation (5.3) with residual:

$$
\begin{aligned}
& r_{\text {inner }}^{\epsilon}(x, t)=\epsilon^{-1 / 4} \sum_{\sigma= \pm} e^{i\left\{S_{\sigma}(t)+\epsilon^{1 / 2} p_{\sigma}(t) y_{\sigma}\right\} / \epsilon}\left\{\epsilon^{3 / 2}\left[y_{\sigma}^{3} \int_{0}^{1} \frac{(\tau-1)^{3}}{3 !} \partial_{x}^{3} W\left(q_{\sigma}(t)+\tau \epsilon^{1 / 2} y_{\sigma}\right) \mathrm{d} \tau\right]\right. \\
& +\epsilon\left[\frac{1}{2}\left(-i \partial_{y_{\sigma}}\right)^{2}+\frac{1}{2} \partial_{x}^{2} W\left(q_{\sigma}(t)\right) y_{\sigma}^{2}-i \partial_{t}\right]+\epsilon^{1 / 2}\left[\left(p_{\sigma}(t)-i \partial_{z}-\partial_{p} E_{\sigma}\left(p_{\sigma}(t)\right)\right)\left(-i \partial_{y_{\sigma}}\right)-i \partial_{s}\right] \\
& \left.+\left[H\left(p_{\sigma}(t)\right)-E_{\sigma}\left(p_{\sigma}(t)\right)\right]\right\}\left.\left\{f_{\sigma, \text { inner }}^{0}\left(y_{\sigma}, z, t, s\right)+\epsilon^{1 / 2} f_{\sigma, \text { inner }}^{1}\left(y_{\sigma}, z, t, s\right)+\ldots\right\}\right|_{y_{\sigma}=\frac{x-q_{\sigma}(t)}{\epsilon^{1 / 2}}, z=\frac{x}{\epsilon}, s=\frac{t-t^{*}}{\epsilon^{1 / 2}}} \\
& =r_{\text {inner }, 0}^{\epsilon}(x, t)+\epsilon^{1 / 2} r_{\text {inner }, 1}^{\epsilon}(x, t)+\left(\epsilon^{1 / 2}\right)^{2} r_{\text {inner }, 2}^{\epsilon}(x, t)+\ldots+\left(\epsilon^{1 / 2}\right)^{m} r_{\text {inner }, 3}^{\epsilon}(x, t)+\ldots
\end{aligned}
$$

Here, $H(p)=-\frac{1}{2}\left(p-i \partial_{z}\right)^{2}+V(z)$; see 2.3).

In the coming subsections we construct the functions $f_{\sigma, i n n e r}^{j}$ so that $\psi_{a p p, i n n e r}^{\epsilon}(x, t)$ satisfies the properties (P1), (P2) and (P3) of Proposition 5.1.

5.1.1. Terms in $r_{\text {inner }}^{\epsilon}(x, t)$ with $L_{x}^{2}$ norm of order $\epsilon^{0}$. The terms with $L_{x}^{2}$ norm proportional to $\epsilon^{0}=1$ in 5.15 are of the form:

$r_{\text {inner }, 0}^{\epsilon}(x, t)=\left.\epsilon^{-1 / 4} \sum_{\sigma= \pm} e^{i\left\{S_{\sigma}(t)+\epsilon^{1 / 2} p_{\sigma}(t) y_{\sigma}\right\} / \epsilon}\left[H\left(p_{\sigma}(t)\right)-E_{\sigma}\left(p_{\sigma}(t)\right)\right] f_{\sigma, \text { inner }}^{0}\left(y_{\sigma}, z, t, s\right)\right|_{y_{\sigma}=\frac{x-q_{\sigma}(t)}{\epsilon^{1 / 2}}, z=\frac{x}{\epsilon}, s=\frac{t-t^{*}}{\epsilon^{1 / 2}}}$.

We may set these two terms individually to zero by defining:

$$
f_{\sigma, i n n e r}^{0}\left(y_{\sigma}, z, t, s\right)=a_{\sigma, i n n e r}^{0}\left(y_{\sigma}, t, s\right) \chi_{\sigma}\left(z ; p_{\sigma}(t)\right), \quad \sigma= \pm \quad .
$$

The functions $a_{\sigma, \text { inner }}^{0}\left(y_{\sigma}, t, s\right), \sigma= \pm$ are left arbitrary for now and will be determined at a later stage.

5.1.2. Terms in $r_{i n n e r}^{\epsilon}$ with $L_{x}^{2}$ norm of order $\epsilon^{1 / 2}$. The terms with $L_{x}^{2}$ norm proportional to $\epsilon^{1 / 2}$ in (5.15) are of the form $\epsilon^{1 / 2}$ times the following expression which is $O_{L^{2}}(1)$ :

$$
\begin{aligned}
& r_{\text {inner }, 1}^{\epsilon}(x, t)=\epsilon^{-1 / 4} \sum_{\sigma= \pm} e^{i\left\{S_{\sigma}(t)+\epsilon^{1 / 2} p_{\sigma}(t) y_{\sigma}\right\} / \epsilon}\{ \\
& {\left[\left(p_{\sigma}(t)-i \partial_{z}-\partial_{p} E_{\sigma}\left(p_{\sigma}(t)\right)\right)\left(-i \partial_{y_{\sigma}}\right)-i \partial_{s}\right] f_{\sigma, \text { inner }}^{0}\left(y_{\sigma}, z, t, s\right)} \\
& \left.+\left[H\left(p_{\sigma}(t)\right)-E_{\sigma}\left(p_{\sigma}(t)\right)\right] f_{\sigma, \text { inner }}^{1}\left(y_{\sigma}, z, t, s\right)\right\}\left.\right|_{y_{\sigma}=\frac{x-q_{\sigma}(t)}{\epsilon^{1 / 2}}, z=\frac{x}{\epsilon}, s=\frac{t-t^{*}}{\epsilon^{1 / 2}}}
\end{aligned}
$$


Proposition 5.2. Substituting the expression (5.17) for $f_{\sigma, \text { inner }}^{0}\left(y_{\sigma}, z, t, s\right)$ into (5.18) yields the following equivalent expression for (5.18):

$$
\begin{aligned}
& \epsilon^{-1 / 4} \sum_{\sigma= \pm} e^{i\left\{S_{\sigma}(t)+\epsilon^{1 / 2} p_{\sigma}(t) y_{\sigma}\right\} / \epsilon}\left\{[ H ( p _ { \sigma } ( t ) ) - E _ { \sigma } ( p _ { \sigma } ( t ) ) ] \left(f_{\sigma, i n n e r}^{1}\left(y_{\sigma}, z, t, s\right)\right.\right. \\
& \left.\left.-\left(-i \partial_{y_{\sigma}}\right) a_{\sigma, i n n e r}^{0}\left(y_{\sigma}, t, s\right) \partial_{p_{\sigma}} \chi_{\sigma}\left(z ; p_{\sigma}(t)\right)\right)-i \partial_{s} a_{\sigma, i n n e r}^{0}\left(y_{\sigma}, s\right) \chi_{\sigma}\left(z ; p_{\sigma}(t)\right)\right\}\left.\right|_{y_{\sigma}=\frac{x-q_{\sigma}(t)}{\epsilon^{1 / 2}, z=\frac{x}{\epsilon}, s=\frac{t-t^{*}}{\epsilon^{1 / 2}}}} .
\end{aligned}
$$

Proof. Differentiating the eigenvalue problem 2.3 satisfied by $\left(E_{\sigma}, \chi_{\sigma}\right)$ with respect to $p$, we obtain the following pair of identities for $\sigma= \pm$ :

$$
\begin{aligned}
& \left(p_{\sigma}(t)-i \partial_{z}-\partial_{p_{\sigma}} E_{\sigma}\left(p_{\sigma}(t)\right)\right) \chi_{\sigma}\left(z ; p_{\sigma}(t)\right) \\
& =-\left(H\left(p_{\sigma}(t)\right)-E_{\sigma}\left(p_{\sigma}(t)\right)\right) \partial_{p} \chi_{\sigma}\left(z ; p_{\sigma}(t)\right) .
\end{aligned}
$$

Relation 5.19) now follows from substituting (5.17) into (5.18) and using (5.20).

We may therefore set the expression in (5.18) equal to zero by setting each term in the sum individually to zero. To do this, we first take:

$$
\partial_{s} a_{\sigma, i n n e r}^{0}\left(y_{\sigma}, t, s\right)=0, \quad \sigma= \pm .
$$

We then require, for $\sigma \in \pm$, that $f_{\sigma, i n n e r}^{1}\left(y_{\sigma}, z, t, s\right)$ satisfy:

$$
\left[H\left(p_{\sigma}(t)\right)-E_{\sigma}\left(p_{\sigma}(t)\right)\right]\left(f_{\sigma, i n n e r}^{1}\left(y_{\sigma}, z, t, s\right)-\left(-i \partial_{y_{\sigma}}\right) a_{\sigma, i n n e r}^{0}\left(y_{\sigma}, t, s\right) \partial_{p_{\sigma}} \chi_{\sigma}\left(z ; p_{\sigma}(t)\right)\right)=0
$$

Therefore, for $\sigma= \pm$,

$f_{\sigma, \text { inner }}^{1}\left(y_{\sigma}, z, t, s\right)=a_{\sigma, \text { inner }}^{1}\left(y_{\sigma}, t, s\right) \chi_{\sigma}\left(z ; p_{\sigma}(t)\right)+\left(-i \partial_{y_{\sigma}}\right) a_{\sigma, \text { inner }}^{0}\left(y_{\sigma}, t\right) \partial_{p} \chi_{\sigma}\left(z ; p_{\sigma}(t)\right)$,

where the functions $a_{\sigma, \text { inner }}^{1}\left(y_{\sigma}, t, s\right)$ are thus far arbitrary and to be determined.

5.1.3. Terms in $r_{\text {inner }}^{\epsilon}$ with $L_{x}^{2}$ norm of order $\epsilon^{1}$. The terms in $r_{i n n e r}^{\epsilon}$ with $L_{x}^{2}$ norm proportional to $\epsilon^{1}$ in 5.15) are of the form: $\epsilon$ times the following expression which is $O_{L^{2}}(1)$ :

$$
\begin{aligned}
& r_{\text {inner }, 2}^{\epsilon}(x, t)=\epsilon^{-1 / 4} \sum_{\sigma= \pm} e^{i\left\{S_{\sigma}(t)+\epsilon^{1 / 2} p_{\sigma}(t) y_{\sigma}\right\} / \epsilon}\{ \\
& +\left[\frac{1}{2}\left(-i \partial_{y_{\sigma}}\right)^{2}+\frac{1}{2} \partial_{x}^{2} W\left(q_{\sigma}(t)\right) y_{\sigma}^{2}-i \partial_{t}\right] f_{\sigma, \text { inner }}^{0}\left(y_{\sigma}, z, t, s\right) \\
& +\left[\left(p_{\sigma}(t)-i \partial_{z}-\partial_{p} E_{\sigma}\left(p_{\sigma}(t)\right)\right)\left(-i \partial_{y_{\sigma}}\right)-i \partial_{s}\right] f_{\sigma, i n n e r}^{1}\left(y_{\sigma}, z, t, s\right) \\
& \left.+\left[H\left(p_{\sigma}(t)\right)-E_{\sigma}\left(p_{\sigma}(t)\right)\right] f_{\sigma, \text { inner }}^{2}\left(y_{\sigma}, z, t, s\right)\right\}\left.\right|_{y_{\sigma}=\frac{x-q_{\sigma}(t)}{\epsilon^{\sigma / 2}}, z=\frac{x}{\epsilon}, s=\frac{t-t^{*}}{\epsilon^{1 / 2}}}
\end{aligned}
$$

Recall (Proposition 5.1 (P2)) that we must choose the $f_{\sigma, \text { inner }}^{j}$ in 5.15 such that $\frac{1}{\epsilon} \int_{t^{*}-\epsilon^{\xi^{\prime}}}^{t^{*}+\epsilon^{\xi^{\prime}}}\left\|r_{\text {inner }}^{\epsilon}\left(\cdot, t^{\prime}\right)\right\|_{L^{2}} \mathrm{~d} t^{\prime}=o\left(\epsilon^{1 / 2}\right)$ for all $t=\left(t^{*}-\epsilon^{\xi^{\prime}}, t^{*}+\epsilon^{\xi^{\prime}}\right)$. It follows that we need to choose the undetermined functions so that $r_{\text {inner, },}^{\epsilon}(x, t)$ in 5.24 satisfies:

$$
\int_{t^{*}-\epsilon^{\xi}}^{t^{*}+\epsilon^{\xi^{\prime}}}\left\|r_{\text {inner }, 2}^{\epsilon}(\cdot, t)\right\|_{L^{2}} \mathrm{~d} t=o\left(\epsilon^{1 / 2}\right) .
$$


In contrast to considerations at previous orders in $\epsilon^{1 / 2}$, we will not be able to satisfy (5.25) by choosing each summand of (5.24) to satisfy the smallness condition (5.25). To see this and to see how to proceed, we first simplify the expression (5.24) using the expressions for $f_{\sigma, \text { inner }}^{0}\left(y_{\sigma}, z, t, s\right)$ 5.17) and $f_{\sigma, \text { inner }}^{1}\left(y_{\sigma}, z, t, s\right) 5.23$ derived above.

Proposition 5.3. The expression (5.24) may be written in the following form:

$$
\begin{aligned}
& r_{i n n e r, 2}^{\epsilon}(x, t) \\
& =\epsilon^{-1 / 4} \sum_{\sigma= \pm} e^{i\left\{S_{\sigma}(t)+\epsilon^{1 / 2} p_{\sigma}(t) y_{\sigma}\right\} / \epsilon}\left\{[ H ( p _ { \sigma } ( t ) ) - E _ { \sigma } ( p _ { \sigma } ( t ) ) ] \left(f_{\sigma, \text { inner }}^{2}\left(y_{\sigma}, z, t, s\right)\right.\right. \\
& \left.-\left(-i \partial_{y_{\sigma}}\right) a_{\sigma, \text { inner }}^{1}\left(y_{\sigma}, t, s\right) \partial_{p_{\sigma}} \chi_{\sigma}\left(z ; p_{\sigma}(t)\right)-\frac{1}{2}\left(-i \partial_{y_{\sigma}}\right)^{2} a_{\sigma, i n n e r}^{0}\left(y_{\sigma}, t\right) \partial_{p_{\sigma}}^{2} \chi_{\sigma}\left(z ; p_{\sigma}(t)\right)\right) \\
& -i \partial_{s} a_{\sigma, i n n e r}^{1}\left(y_{\sigma}, t, s\right) \chi_{\sigma}\left(z ; p_{\sigma}(t)\right)-\left[i \partial_{t}-\mathscr{H}_{\sigma}(t)\right] a_{\sigma, i n n e r}^{0}\left(y_{\sigma}, t\right) \chi_{\sigma}\left(z ; p_{\sigma}(t)\right) \\
& +i \partial_{q_{\sigma}} W\left(q_{\sigma}(t)\right) a_{\sigma, \text { inner }}^{0}\left(y_{\sigma}, t, s\right)\left\langle\chi_{-\sigma}\left(\cdot ; p_{\sigma}(t)\right) \mid \partial_{p_{\sigma}} \chi_{\sigma}\left(\cdot ; p_{\sigma}(t)\right)\right\rangle \chi_{-\sigma}\left(z ; p_{\sigma}(t)\right) \\
& \left.+i \partial_{q_{\sigma}} W\left(q_{\sigma}(t)\right) a_{\sigma, \text { inner }}^{0}\left(y_{\sigma}, t, s\right) P_{ \pm}^{\perp}\left(p_{\sigma}(t)\right) \partial_{p_{\sigma}} \chi_{\sigma}\left(z ; p_{\sigma}(t)\right)\right\}\left.\right|_{y_{\sigma}=\frac{x-q_{\sigma}(t)}{\epsilon^{1 / 2}}, z=\frac{x}{\epsilon}, s=\frac{t-t^{*}}{\epsilon^{1 / 2}}}
\end{aligned}
$$

Here, we recall that $H(p)=-\frac{1}{2}\left(p-i \partial_{z}\right)^{2}+V(z)$ and $\mathscr{H}_{\sigma}(t)$ denotes the timedependent harmonic oscillator Hamiltonian defined in (2.14), where we replace $p(t), q(t), E_{n}, \chi_{n}, y$, respectively, by $p_{\sigma}(t), q_{\sigma}(t), E_{\sigma}, \chi_{\sigma}, y_{\sigma}$. Finally, $P_{ \pm}^{\perp}\left(p_{\sigma}(t)\right)$ denotes the orthogonal projection operator given by:

$$
P_{ \pm}^{\perp}\left(p_{\sigma}(t)\right) f(z):=f(z)-\sum_{\sigma^{\prime}= \pm}\left\langle\chi_{\sigma^{\prime}}\left(\cdot ; p_{\sigma}(t)\right) \mid f(\cdot)\right\rangle \chi_{\sigma^{\prime}}\left(z ; p_{\sigma}(t)\right) .
$$

Proof. We begin with the identity, obtained by differentiating the eigenvalue problem (2.3), satisfied by the eigenpair $\left(E_{\sigma}, \chi_{\sigma}\right)$, twice with respect to $p$ :

$$
\begin{aligned}
& \frac{1}{2}\left(1-\partial_{p}^{2} E_{\sigma}\left(p_{\sigma}(t)\right)\right) \chi_{\sigma}\left(z ; p_{\sigma}(t)\right)+\left(p_{\sigma}(t)-i \partial_{z}-\partial_{p_{\sigma}} E_{\sigma}\left(p_{\sigma}(t)\right)\right) \partial_{p_{\sigma}} \chi_{\sigma}\left(z ; p_{\sigma}(t)\right) \\
& =-\frac{1}{2}\left(H\left(p_{\sigma}(t)\right)-E_{\sigma}\left(p_{\sigma}(t)\right)\right) \partial_{p_{\sigma}}^{2} \chi_{\sigma}\left(z ; p_{\sigma}(t)\right), \quad \sigma= \pm .
\end{aligned}
$$

To obtain the expression (5.26), we first substitute expression (5.17) for $f_{\sigma, \text { inner }}^{0}$ and expression (5.23) for $f_{\sigma, \text { inner }}^{1}$ into 5.24 . We then simplify using the identity (5.28) and the expansion of $\partial_{p_{\sigma}} \chi_{\sigma}\left(z ; p_{\sigma}(t)\right)$ in terms of its orthogonal components:

$$
\begin{aligned}
& \partial_{p} \chi_{\sigma}\left(z ; p_{\sigma}(t)\right)= \\
& \sum_{\sigma^{\prime}= \pm}\left\langle\chi_{\sigma^{\prime}}\left(\cdot ; p_{\sigma}(t)\right) \mid \partial_{p} \chi_{\sigma}\left(\cdot ; p_{\sigma}(t)\right)\right\rangle \chi_{\sigma^{\prime}}\left(z ; p_{\sigma}(t)\right)+P_{ \pm}^{\perp}\left(p_{\sigma}(t)\right) \chi_{\sigma}\left(z ; p_{\sigma}(t)\right) .
\end{aligned}
$$

By Proposition 5.3 the smallness condition 5.25 may be studied with the expression (5.26) in place of (5.24). We proceed in two steps.

(A) We first use certain degrees of freedom to eliminate 'in-band' contributions to 5.26 . 
(B) We will then be left with contributions which relate to the coupling of bands revealed in analysis of the breakdown of the single-band approximation.

Step A: We first choose $a_{\sigma, \text { inner }}^{0}\left(y_{\sigma}, t\right)$ so that:

$$
i \partial_{t} a_{\sigma, \text { inner }}^{0}\left(y_{\sigma}, t\right)=\mathscr{H}_{\sigma}(t) a_{\sigma, \text { inner }}^{0}\left(y_{\sigma}, t\right), \quad \sigma= \pm .
$$

We also require that $z \mapsto f_{\sigma, i n n e r}^{2}\left(y_{\sigma}, z, t, s\right)$ be a $1-$ periodic solution of:

$$
\begin{aligned}
& {\left[H\left(p_{\sigma}(t)\right)-E_{\sigma}\left(p_{\sigma}(t)\right)\right]\left(f_{\sigma, \text { inner }}^{2}\left(y_{\sigma}, z, t, s\right)\right.} \\
& \left.-\left(-i \partial_{y_{\sigma}}\right) a_{\sigma, \text { inner }}^{1}\left(y_{\sigma}, t, s\right) \partial_{p_{\sigma}} \chi_{\sigma}\left(z ; p_{\sigma}(t)\right)-\frac{1}{2}\left(-i \partial_{y_{\sigma}}\right)^{2} a_{\sigma, \text { inner }}^{0}\left(y_{\sigma}, t\right) \partial_{p_{\sigma}}^{2} \chi_{\sigma}\left(z ; p_{\sigma}(t)\right)\right) \\
& =-i \partial_{q_{\sigma}} W\left(q_{\sigma}(t)\right) a_{\sigma, \text { inner }}^{0}\left(y_{\sigma}, t, s\right) P_{ \pm}^{\perp}\left(p_{\sigma}(t)\right) \partial_{p_{\sigma}} \chi_{\sigma}\left(z ; p_{\sigma}(t)\right) .
\end{aligned}
$$

Equation (5.31) is solvable, with a uniform bound in time on the inverse, for all $t$ near $t^{*}$ by Corollary 3.3 . Hence we have for $\sigma= \pm$ :

$$
\begin{aligned}
& f_{\sigma, \text { inner }}^{2}\left(y_{\sigma}, z, t, s\right)=a_{\sigma, \text { inner }}^{2}\left(y_{\sigma}, t, s\right) \chi_{\sigma}\left(z ; p_{\sigma}(t)\right) \\
& +\left(-i \partial_{y_{\sigma}}\right) a_{\sigma, \text { inner }}^{1}\left(y_{\sigma}, s, t\right) \partial_{p} \chi_{\sigma}\left(z ; p_{\sigma}(t)\right)+\frac{1}{2}\left(-i \partial_{y_{\sigma}}\right)^{2} a_{\sigma, \text { inner }}^{0}\left(y_{\sigma}, t\right) \partial_{p}^{2} \chi_{\sigma}\left(z ; p_{\sigma}(t)\right) \\
& -i \partial_{q} W\left(q_{\sigma}(t)\right) a_{\sigma, \text { inner }}^{0}\left(y_{\sigma}, t, s\right) \mathcal{R}_{\sigma}\left(p_{\sigma}(t)\right) P_{ \pm}^{\perp}\left(p_{\sigma}(t)\right) \partial_{p} \chi_{\sigma}\left(z ; p_{\sigma}(t)\right) .
\end{aligned}
$$

Here, $a_{\sigma, \text { inner }}^{2}\left(y_{\sigma}, t, s\right)$ is presently arbitrary and can be determined at higher order in $\epsilon^{1 / 2}$.

The initial data for equations 5.30 is fixed by the requirement that $\psi_{a p p, i n n e r}^{\epsilon}$ satisfy (P1) of Proposition 5.1. By inspection of the incoming solution, we see that this is equivalent to requiring that:

$$
\text { for } t \in\left(t^{*}-\epsilon^{\xi^{\prime}}, t^{*}-\epsilon^{\xi}\right): a_{+, \text {inner }}^{0}(y, t)=a_{+}^{0}(y, t) \text { and } a_{-, \text {inner }}(y, t)=0 \text {. }
$$

The only choice of initial data $a_{+, \text {inner }}^{0}\left(y, t^{*}\right), a_{-, \text {inner }}^{0}\left(y, t^{*}\right)$ for 5.30 consistent with $(5.33)$ are :

$$
a_{+, \text {inner }}^{0}\left(y, t^{*}\right)=a_{+}^{0}\left(y, t^{*}\right)=a^{0, *}(y), \quad a_{-, \text {inner }}^{0}\left(y, t^{*}\right)=0 .
$$

Here, $a^{0, *}(y):=\lim _{t \uparrow t^{*}} a^{0}(y, t)(3.22)$. Indeed, for all $t \in\left(t^{*}-\epsilon^{\xi^{\prime}}, t^{*}+\epsilon^{\xi^{\prime}}\right)$ :

$$
a_{+, \text {inner }}^{0}\left(y_{+}, t\right)=a_{+}\left(y_{+}, t\right) \text {, and } a_{-, \text {inner }}^{0}\left(y_{-}, t\right)=0 .
$$

The choices 5.30, 5.32), and 5.34 simplify (5.26) to:

$$
\begin{aligned}
& r_{\text {inner }, 2}^{\epsilon}(x, t)=\epsilon^{-1 / 4} e^{i\left\{S_{+}(t)+\epsilon^{1 / 2} p_{+}(t) y_{+}\right\} / \epsilon}\left\{-i \partial_{s} a_{+, \text {inner }}^{1}\left(y_{+}, t, s\right) \chi_{+}\left(z ; p_{+}(t)\right)\right. \\
& \left.+i \partial_{q_{+}} W\left(q_{+}(t)\right) a_{+, \text {inner }}^{0}\left(y_{+}, t, s\right)\left\langle\chi_{-}\left(\cdot ; p_{+}(t)\right) \mid \partial_{p_{+}} \chi_{+}\left(\cdot ; p_{+}(t)\right)\right\rangle \chi_{-}\left(z ; p_{+}(t)\right)\right\} \\
& \left.\epsilon^{-1 / 4} e^{i\left\{S_{-}(t)+\epsilon^{1 / 2} p_{-}(t) y_{-}\right\} / \epsilon}\left\{-i \partial_{s} a_{-, \text {inner }}^{1}\left(y_{-}, t, s\right) \chi_{-}\left(z ; p_{-}(t)\right)\right\}\right|_{y_{\sigma}=\frac{x-q_{\sigma}(t)}{\epsilon^{1 / 2}}, z=\frac{x}{\epsilon}, s=\frac{t-t^{*}}{\epsilon^{1 / 2}}}
\end{aligned}
$$


We find that at this order in $\epsilon^{1 / 2}$ that there is no loss in taking the functions $a_{\sigma, i n n e r}^{1}, \sigma= \pm$ to be independent of $t$ :

$$
a_{+, \text {inner }}^{1}\left(y_{+}, t, s\right)=a_{+, \text {inner }}^{1}\left(y_{+}, s\right), \quad a_{-, \text {inner }}^{1}\left(y_{-}, t, s\right)=a_{-, \text {inner }}^{1}\left(y_{-}, s\right) .
$$

From 5.35 it is natural to set:

$$
\begin{aligned}
& \partial_{s} a_{+, \text {inner }}^{1}\left(y_{+}, s\right)=0 \\
& a_{+, \text {inner }}^{1}\left(y_{+}, 0\right)=a_{+, \text {inner }, 0}^{1}\left(y_{+}\right) .
\end{aligned}
$$

and to choose $a_{-, \text {inner }}^{1}\left(y_{+}, s\right)$ to eliminate the projection of $r_{\text {inner, } 2}^{\epsilon}(x, t)$ onto the vector $\chi_{-}\left(z ; p_{+}(t)\right)$. The function $a_{+, \text {inner }, 0}^{1}\left(y_{+}\right)$is at this point arbitrary, it will be fixed below by enforcing (P1) of Proposition 5.1 .

Taking $a_{+, \text {inner }}^{1}\left(y_{+}, s\right)$ to satisfy (5.36) reduces (5.35) to the following:

$$
\begin{aligned}
& r_{\text {inner }, 2}^{\epsilon}(x, t)=\epsilon^{-1 / 4} e^{i\left\{S_{+}(t)+\epsilon^{1 / 2} p_{+}(t) y_{+}\right\} / \epsilon}[ \\
& \left.i \partial_{q} W\left(q_{+}(t)\right) a_{+}\left(y_{+}, t\right)\left\langle\chi_{-}\left(\cdot ; p_{+}(t)\right) \mid \partial_{p} \chi_{+}\left(\cdot ; p_{+}(t)\right)\right\rangle \chi_{-}\left(z ; p_{+}(t)\right)\right] \\
& +\left.\epsilon^{-1 / 4} e^{i\left\{S_{-}(t)+\epsilon^{1 / 2} p_{-}(t) y_{-}\right\} / \epsilon}\left[-i \partial_{s} a_{-, \text {inner }}^{1}\left(y_{-}, s\right) \chi_{-}\left(z ; p_{-}(t)\right)\right]\right|_{y_{\sigma}=\frac{x-q_{\sigma}(t)}{\epsilon^{1 / 2}}, z=\frac{x}{\epsilon}, s=\frac{t-t^{*}}{\epsilon^{1 / 2}}} .
\end{aligned}
$$

We next determine the evolution of $a_{-, \text {inner }}^{1}\left(y_{-}, s\right)$ to satisfy the smallness condition (5.25). We find it useful at this point to re-express functions of $t$ and $y_{+}$in terms of the variables $y_{-}$and $s$ using the relations:

$$
y_{+}=y_{-}+\frac{q_{-}(t)-q_{+}(t)}{\epsilon^{1 / 2}}, \quad t=t^{*}+\epsilon^{1 / 2} s
$$

This yields:

$$
\begin{aligned}
& r_{\text {inner }, 2}^{\epsilon}\left(x, t^{*}+\epsilon^{1 / 2} s\right) \\
& =\epsilon^{-1 / 4} e^{i\left\{S_{+}\left(t^{*}+\epsilon^{1 / 2} s\right)+\epsilon^{1 / 2} p_{+}\left(t^{*}+\epsilon^{1 / 2} s\right) y_{-}+p_{+}\left(t^{*}+\epsilon^{1 / 2} s\right)\left(q_{-}\left(t^{*}+\epsilon^{1 / 2} s\right)-q_{+}\left(t^{*}+\epsilon^{1 / 2} s\right)\right)\right\} / \epsilon}[ \\
& i \partial_{q} W\left(q_{+}\left(t^{*}+\epsilon^{1 / 2} s\right)\right) a_{+}\left(y_{-}+\frac{q_{-}\left(t^{*}+\epsilon^{1 / 2} s\right)-q_{+}\left(t^{*}+\epsilon^{1 / 2} s\right)}{\epsilon^{1 / 2}}, t^{*}+\epsilon^{1 / 2} s\right) \\
& \left.\times\left\langle\chi_{-}\left(\cdot ; p_{+}\left(t^{*}+\epsilon^{1 / 2} s\right)\right) \mid \partial_{p} \chi_{+}\left(\cdot ; p_{+}\left(t^{*}+\epsilon^{1 / 2} s\right)\right)\right\rangle \chi_{-}\left(z ; p_{+}\left(t^{*}+\epsilon^{1 / 2} s\right)\right)\right] \\
& +\epsilon^{-1 / 4} e^{i\left\{S_{-}\left(t^{*}+\epsilon^{1 / 2} s\right)+\epsilon^{1 / 2} p_{-}\left(t^{*}+\epsilon^{1 / 2} s\right) y_{-}\right\} / \epsilon}[ \\
& \left.-i \partial_{s} a_{-, i n n e r}^{1}\left(y_{-}, s\right) \chi_{-}\left(z ; p_{-}\left(t^{*}+\epsilon^{1 / 2} s\right)\right)\right]\left.\right|_{y_{-}=\frac{x-q_{-}\left(t^{*}+\epsilon^{1 / 2} s\right)}{\epsilon^{1 / 2}}, z=\frac{x}{\epsilon}} .
\end{aligned}
$$

In terms of $s$, the condition 5.25 reads:

$$
\int_{-\epsilon^{\xi^{\prime}-1 / 2}}^{\epsilon^{\xi^{\prime}-1 / 2}}\left\|r_{\text {inner }, 2}^{\epsilon}\left(\cdot, t^{*}+\epsilon^{1 / 2} s\right)\right\|_{L^{2}}=o(1) .
$$

We proceed with the construction of $a_{-, \text {inner }}^{1}\left(y_{-}, s\right)$ by seeking the expression in $r_{\text {inner }, 2}^{\epsilon}\left(x, t^{*}+\epsilon^{1 / 2} s\right)$ which, to leading order, will be balanced (indeed cancelled out by) the term proportional to $\partial_{s} a_{-, \text {inner }}^{1}\left(y_{-}, s\right)$, for $-\epsilon^{\xi^{\prime}-1 / 2}<s<\epsilon^{\xi^{\prime}-1 / 2}$ $\left(0<\xi^{\prime}<1 / 2\right)$. 
Thus we expand the expression for $r_{\text {inner, } 2}^{\epsilon}\left(x, t^{*}+\epsilon^{1 / 2} s\right)$ in powers of $\epsilon^{1 / 2} s$, making use of the equations governing $\left(q_{ \pm}(t), p_{ \pm}(t)\right)$ and $S_{ \pm}(t)$ (3.16), 3.35), Definitions 3.10 and 3.18 to compute their derivatives. We first Taylor-expand the expression within square brackets in (5.39):

$$
\begin{aligned}
& r_{\text {inner }, 2}^{\epsilon}\left(x, t^{*}+\epsilon^{1 / 2} s\right) \\
& =\epsilon^{-1 / 4} e^{i\left\{S_{+}\left(t^{*}+\epsilon^{1 / 2} s\right)+\epsilon^{1 / 2} p_{+}\left(t^{*}+\epsilon^{1 / 2} s\right) y_{-}+p_{+}\left(t^{*}+\epsilon^{1 / 2} s\right)\left(q_{-}\left(t^{*}+\epsilon^{1 / 2} s\right)-q_{+}\left(t^{*}+\epsilon^{1 / 2} s\right)\right)\right\} / \epsilon}[ \\
& \left.i \partial_{q} W\left(q^{*}\right) a_{+}\left(y_{-}+\left[\partial_{p} E_{-}\left(p^{*}\right)-\partial_{p} E_{+}\left(p^{*}\right)\right] s, t^{*}\right)\left\langle\chi_{-}\left(\cdot ; p^{*}\right) \mid \partial_{p} \chi_{+}\left(\cdot ; p^{*}\right)\right\rangle \chi_{-}\left(z ; p^{*}\right)\right] \\
& +\left.\epsilon^{-1 / 4} e^{i\left\{S_{-}\left(t^{*}+\epsilon^{1 / 2} s\right)+\epsilon^{1 / 2} p_{-}\left(t^{*}+\epsilon^{1 / 2} s\right) y_{-}\right\} / \epsilon}\left[-i \partial_{s} a_{-, i n n e r}^{1}\left(y_{-}, s\right) \chi_{-}\left(z ; p^{*}\right)\right]\right|_{y_{-}=\frac{x-q_{-}\left(t^{*}+\epsilon^{1 / 2} s\right)}{\epsilon^{1 / 2}}, z=\frac{x}{\epsilon}} \\
& +O_{L_{x}^{2}}\left(\epsilon^{1 / 2} s, \epsilon^{1 / 2} s^{2}\right) .
\end{aligned}
$$

Rearranging terms, we obtain:

$$
\begin{aligned}
& r_{\text {inner }, 2}^{\epsilon}\left(x, t^{*}+\epsilon^{1 / 2} s\right) \\
& =\epsilon^{-1 / 4} e^{i\left\{S_{-}\left(t^{*}+\epsilon^{1 / 2} s\right)+\epsilon^{1 / 2} p_{-}\left(t^{*}+\epsilon^{1 / 2} s\right) y_{-}\right\} / \epsilon} i \chi_{-}\left(z ; p^{*}\right) \\
& \times\left\{e^{i\left\{S_{+}\left(t^{*}+\epsilon^{1 / 2} s\right)-S_{-}\left(t^{*}+\epsilon^{1 / 2} s\right)+\epsilon^{1 / 2}\left(p_{+}\left(t^{*}+\epsilon^{1 / 2} s\right)-p_{-}\left(t^{*}+\epsilon^{1 / 2} s\right)\right) y_{-}+p_{+}\left(t^{*}+\epsilon^{1 / 2} s\right)\left(q_{-}\left(t^{*}+\epsilon^{1 / 2} s\right)-q_{+}\left(t^{*}+\epsilon^{1 / 2} s\right)\right)\right\} / \epsilon}\right. \\
& \times\left[\partial_{q} W\left(q^{*}\right) a_{+}\left(y_{-}+\left[\partial_{p} E_{-}\left(p^{*}\right)-\partial_{p} E_{+}\left(p^{*}\right)\right] s, t^{*}\right)\left\langle\chi_{-}\left(\cdot ; p^{*}\right) \mid \partial_{p} \chi_{+}\left(\cdot ; p^{*}\right)\right\rangle\right] \\
& \left.-\partial_{s} a_{-, \text {inner }}^{1}\left(y_{-}, s\right)\right\}\left.\right|_{y_{-}=\frac{x-q_{-}\left(t^{*}+\epsilon^{1 / 2} s\right)}{\epsilon^{1 / 2}}, z=\frac{x}{\epsilon}} \\
& +O_{L_{x}^{2}}\left(\epsilon^{1 / 2} s, \epsilon^{1 / 2} s^{2}\right) .
\end{aligned}
$$

We next Taylor-expand the exponential:

$$
\begin{aligned}
& S_{+}\left(t^{*}+\epsilon^{1 / 2} s\right)-S_{-}\left(t^{*}+\epsilon^{1 / 2} s\right) \\
& =\left(\epsilon^{1 / 2} s\right) p^{*}\left(\partial_{p} E_{+}\left(p^{*}\right)-\partial_{p} E_{-}\left(p^{*}\right)\right) \\
& +\frac{1}{2}\left(\epsilon^{1 / 2} s\right)^{2}\left(\partial_{q} W\left(q^{*}\right) p^{*}\left(\partial_{p}^{2} E_{-}\left(p^{*}\right)-\partial_{p}^{2} E_{+}\left(p^{*}\right)\right)+\partial_{q} W\left(q^{*}\right)\left(\partial_{p} E_{-}\left(p^{*}\right)-\partial_{p} E_{+}\left(p^{*}\right)\right)\right) \\
& +O\left(\epsilon^{3 / 2} s^{3}\right) \\
& \epsilon^{1 / 2}\left(p_{+}\left(t^{*}+\epsilon^{1 / 2} s\right)-p_{-}\left(t^{*}+\epsilon^{1 / 2} s\right)\right) y_{-} \\
& =O\left(\epsilon^{3 / 2} s^{2} y-\right) \\
& p_{+}\left(t^{*}+\epsilon^{1 / 2} s\right)\left(q_{-}\left(t^{*}+\epsilon^{1 / 2} s\right)-q_{+}\left(t^{*}+\epsilon^{1 / 2} s\right)\right) \\
& =\left(\epsilon^{1 / 2} s\right)\left(p^{*}\left(\partial_{p} E_{-}\left(p^{*}\right)-\partial_{p} E_{+}\left(p^{*}\right)\right)\right) \\
& +\frac{1}{2}\left(\epsilon^{1 / 2} s\right)^{2}\left(-2 \partial_{q} W\left(q^{*}\right)\left(\partial_{p} E_{-}\left(p^{*}\right)-\partial_{p} E_{+}\left(p^{*}\right)\right)+\partial_{q} W\left(q^{*}\right) p^{*}\left(\partial_{p}^{2} E_{+}\left(p^{*}\right)-\partial_{p}^{2} E_{-}\left(p^{*}\right)\right)\right) \\
& +O\left(\epsilon^{3 / 2} s^{3}\right) .
\end{aligned}
$$


Substituting these expressions and using the fact that $a_{+}(y, t) \in \mathcal{S}(\mathbb{R})$ gives:

$$
\begin{aligned}
& r_{\text {inner }, 2}^{\epsilon}\left(x, t^{*}+\epsilon^{1 / 2} s\right)=\epsilon^{-1 / 4} e^{i\left\{S_{-}\left(t^{*}+\epsilon^{1 / 2} s\right)+\epsilon^{1 / 2} p_{-}\left(t^{*}+\epsilon^{1 / 2} s\right) y_{-}\right\} / \epsilon} i \chi_{-}\left(z ; p^{*}\right) \\
& \times\left\{e^{i \frac{1}{2} \partial_{q} W\left(q^{*}\right)\left(\partial_{p} E_{+}\left(p^{*}\right)-\partial_{p} E_{-}\left(p^{*}\right)\right) s^{2}}\right. \\
& \times\left[\partial_{q} W\left(q^{*}\right)\left\langle\chi_{-}\left(\cdot ; p^{*}\right) \mid \partial_{p} \chi_{+}\left(\cdot ; p^{*}\right)\right\rangle a_{+}\left(y_{-}+\left[\partial_{p} E_{-}\left(p^{*}\right)-\partial_{p} E_{+}\left(p^{*}\right)\right] s, t^{*}\right)\right] \\
& \left.-\partial_{s} a_{-, \text {inner }}^{1}\left(y_{-}, s\right)\right\}+\left.O_{L_{x}^{2}}\left(\epsilon^{1 / 2} s, \epsilon^{1 / 2} s^{2}, \epsilon^{1 / 2} s^{3}\right)\right|_{y_{-}=\frac{x-q_{-}(t)}{\epsilon^{1 / 2}}, z=\frac{x}{\epsilon}, s=\frac{t-t^{*}}{\epsilon^{1 / 2}}}
\end{aligned}
$$

It follows that by taking $a_{-, \text {inner }}^{1}\left(y_{-}, s\right)$ to satisfy:

$$
\begin{aligned}
& \partial_{s} a_{-, \text {inner }}^{1}\left(y_{-}, s\right)=e^{i \frac{1}{2} \partial_{q} W\left(q^{*}\right)\left(\partial_{p} E_{+}\left(p^{*}\right)-\partial_{p} E_{-}\left(p^{*}\right)\right) s^{2}} \\
& \times \partial_{q} W\left(q^{*}\right) a_{+}\left(y_{-}+\left[\partial_{p} E_{-}\left(p^{*}\right)-\partial_{p} E_{+}\left(p^{*}\right)\right] s, t^{*}\right)\left\langle\chi_{-}\left(\cdot ; p^{*}\right) \mid \partial_{p} \chi_{+}\left(\cdot ; p^{*}\right)\right\rangle \\
& a_{-, \text {inner }}^{1}\left(y_{-}, 0\right)=a_{-, \text {inner }, 0}^{1}\left(y_{-}\right) .
\end{aligned}
$$

We have that $\psi_{\text {app,inner }}^{\epsilon}(x, t)$ satisfies $(5.40)$, and therefore (P2) of Proposition 5.1. provided $3 / 8<\xi^{\prime}<1 / 2$. That is, for $3 / 8<\xi^{\prime}<1 / 2$, we have

$$
\int_{-\epsilon^{\xi^{\prime}-1 / 2}}^{\epsilon^{\xi^{\prime}-1 / 2}}\left\|O_{L_{x}^{2}}\left(\epsilon^{1 / 2} s, \epsilon^{1 / 2} s^{2}, \epsilon^{1 / 2} s^{3}\right)\right\|_{L_{x}^{2}} \mathrm{~d} s=O\left(\epsilon^{2 \xi^{\prime}-1 / 2}, \epsilon^{3 \xi^{\prime}-1}, \epsilon^{4 \xi^{\prime}-3 / 2}\right)=o(1) .
$$

The initial data choices $a_{+, \text {inner, } 0}^{1}\left(y_{+}\right)$and $a_{-, \text {inner }, 0}^{1}\left(y_{-}\right)$are forced by the requirement that $\psi_{a p p, i n n e r}^{\epsilon}(x, t)$ satisfies the matching condition (P1) of Proposition 5.1. Since these terms appear at order $\epsilon^{1 / 2}$ in the asymptotic expansion, for (P1) to hold it is sufficient that for $s \in\left(-\epsilon^{\xi^{\prime}-1 / 2}, t^{*}-\epsilon^{\xi-1 / 2}\right): a_{+, \text {inner }}^{1}\left(y_{+}, s\right)-a_{+}^{1}\left(y_{+}, t^{*}+\right.$ $\left.\epsilon^{1 / 2} s\right)=o_{L_{y_{+}}^{2}}(1)$ and $a_{-, \text {inner }}^{1}\left(y_{-}, s\right)=o_{L_{y_{-}}^{2}}(1)$.

We claim that we may ensure this by taking:

$$
a_{+, \text {inner }, 0}^{1}\left(y_{+}\right)=a_{+}^{1}\left(y_{+}, t^{*}\right)=a^{1, *}\left(y_{+}\right),
$$

(recall: $a^{1, *}(y):=\lim _{t \uparrow t^{*}} a^{1}(y, t)$ 3.22), and:

$$
\begin{aligned}
& a_{-, \text {inner }, 0}^{1}\left(y_{-}\right)=\partial_{q} W\left(q^{*}\right) \times\left\langle\chi_{-}\left(\cdot ; p^{*}\right) \mid \partial_{p} \chi_{+}\left(\cdot ; p^{*}\right)\right\rangle \\
& \times \int_{-\infty}^{0} e^{i \frac{1}{2} \partial_{q} W\left(q^{*}\right)\left(\partial_{p} E_{+}\left(p^{*}\right)-\partial_{p} E_{-}\left(p^{*}\right)\right)\left(s^{\prime}\right)^{2}} a_{+}\left(y_{-}+\left[\partial_{p} E_{-}\left(p^{*}\right)-\partial_{p} E_{+}\left(p^{*}\right)\right] s^{\prime}, t^{*}\right) \mathrm{d} s^{\prime} .
\end{aligned}
$$

This claim follows from Taylor-expansion:

$$
\begin{aligned}
& \text { for all } s \in\left(-\epsilon^{\xi^{\prime}-1 / 2}, t^{*}-\epsilon^{\xi-1 / 2}\right): \\
& a_{+}^{1}\left(y_{+}, t^{*}+\epsilon^{1 / 2} s\right)-a_{+}^{1}\left(y_{+}, t^{*}\right)=O_{L_{y_{+}}^{2}}\left(\epsilon^{1 / 2} s\right)=O_{L_{y_{+}}^{2}}\left(\epsilon^{\xi^{\prime}}\right)=o_{L_{y_{+}}^{2}}(1)
\end{aligned}
$$


since $3 / 8<\xi^{\prime}<1 / 2$ and from integration by parts, which shows that for $s \in$ $\left(-\epsilon^{\xi^{\prime}-1 / 2},-\epsilon^{\xi-1 / 2}\right)$ :

$$
\begin{aligned}
& a_{-, \text {inner }}^{1}\left(y_{-}, s\right)=\int_{-\infty}^{s} e^{i \frac{1}{2} \partial_{q} W\left(q^{*}\right)\left(\partial_{p} E_{+}\left(p^{*}\right)-\partial_{p} E_{-}\left(p^{*}\right)\right)\left(s^{\prime}\right)^{2}} \\
& \times \partial_{q} W\left(q^{*}\right) a_{+}\left(y_{-}+\left[\partial_{p} E_{-}\left(p^{*}\right)-\partial_{p} E_{+}\left(p^{*}\right)\right] s^{\prime}, t^{*}\right)\left\langle\chi_{-}\left(\cdot ; p^{*}\right) \mid \partial_{p} \chi_{+}\left(\cdot ; p^{*}\right)\right\rangle \mathrm{d} s^{\prime} \\
& =O_{L_{y_{-}}^{2}}\left(\epsilon^{1 / 2-\xi}\right) .
\end{aligned}
$$

Since $\xi<1 / 2$ by assumption, we are done.

It remains to show (P3) of Proposition 5.1. But by an identical argument, for $s \in\left(\epsilon^{\xi-1 / 2}, \epsilon^{\xi^{\prime}-1 / 2}\right):$

$$
\begin{aligned}
& a_{-, \text {inner }}^{1}\left(y_{-}, s\right)=\partial_{q} W\left(q^{*}\right) \times\left\langle\chi_{-}\left(\cdot ; p^{*}\right) \mid \partial_{p} \chi_{+}\left(\cdot ; p^{*}\right)\right\rangle \\
& \int_{-\infty}^{\infty} e^{i \frac{1}{2} \partial_{q} W\left(q^{*}\right)\left(\partial_{p} E_{+}\left(p^{*}\right)-\partial_{p} E_{-}\left(p^{*}\right)\right)\left(s^{\prime}\right)^{2}} a_{+}\left(y_{-}+\left[\partial_{p} E_{-}\left(p^{*}\right)-\partial_{p} E_{+}\left(p^{*}\right)\right] s^{\prime}, t^{*}\right) \mathrm{d} s^{\prime} \\
& +O\left(\epsilon^{\xi^{\prime}-1 / 2}\right),
\end{aligned}
$$

so that for $\sigma= \pm$ :

$$
a_{\sigma, \text { inner }}^{1}\left(y_{\sigma}, t^{*}+\epsilon^{1 / 2} s\right)-a_{\sigma}^{1}\left(y_{\sigma}, t^{*}\right)=O_{L_{y_{\sigma}}^{2}}\left(\epsilon^{\xi^{\prime}}\right)=o_{L_{y_{\sigma}}^{2}}(1)
$$

for $t-t^{*}=\epsilon^{1 / 2} s \in\left(\epsilon^{\xi}, \epsilon^{\xi^{\prime}}\right)$. It follows that $\psi_{a p p, i n n e r}^{\epsilon}(x, t)$ so constructed satisfies all hypotheses of Proposition 5.1, and so the proof of Theorem 3.20 is complete.

\section{A. Consistency of Theorem 3.20 with 'Landau-Zener' theory}

In this Appendix we:

(1) Derive the precise magnitude (in $L^{2}$ ) of the 'excited' wavepacket from the main statement of Theorem 3.20 (3.45) in terms of that of the initial, 'incident', wavepacket (Section A.1)

(2) Show how this result is consistent with the solution of a simplified 'LandauZener'-type model (Section A.2.

A.1. Magnitude of wave 'excited' at the crossing. Let $t \in\left(t^{*}, \tilde{T}\right]$. According to the main statement of Theorem 3.20 (3.45), the wave 'excited' at the crossing time $t^{*}$ is of the form:

$$
\begin{aligned}
& \operatorname{WP}_{\text {excited }}^{\epsilon}(x, t):= \\
& \sqrt{\epsilon} \mathrm{WP}^{0, \epsilon}\left[\mathfrak{S}_{-}(t), \mathfrak{q}_{-}(t), \mathfrak{p}_{-}(t), \mathfrak{a}_{-}^{0}(y, t), \mathfrak{X}_{-}\left(z ; \mathfrak{p}_{-}(t)\right)\right](x, t)+o_{L_{x}^{2}}(\sqrt{\epsilon})
\end{aligned}
$$

where the parameters of the excited wavepacket: $\mathfrak{S}_{-}(t), \mathfrak{q}_{-}(t), \mathfrak{p}_{-}(t), \mathfrak{a}_{-}^{0}(y, t)$, $\mathfrak{X}_{-}\left(z ; \mathfrak{p}_{-}(t)\right)$ were defined in Proposition 3.17 and Definition 3.18. The notation $\mathrm{WP}^{0, \epsilon}[\ldots](x, t)$ was defined in 2.20$)$.

Expanding the short-hand notation, (A.1) becomes:

(A.2)

$$
\begin{aligned}
& \operatorname{WP}_{\text {excited }}^{\epsilon}(x, t)= \\
& \sqrt{\epsilon} \times \epsilon^{-1 / 4} e^{i \mathfrak{S}_{-}(t) / \epsilon} e^{i \mathfrak{p}_{-}(t)\left(x-\mathfrak{q}_{-}(t)\right) / \epsilon} \mathfrak{a}_{-}^{0}\left(\frac{x-\mathfrak{q}_{-}(t)}{\sqrt{\epsilon}}, t\right) \mathfrak{X}_{-}\left(\frac{x}{\epsilon} ; \mathfrak{p}_{-}(t)\right)+o_{L_{x}^{2}}(\sqrt{\epsilon}) .
\end{aligned}
$$


Using 1-periodicity in $z$ of the function $\mathfrak{X}_{-}\left(z ; \mathfrak{p}_{-}(t)\right)$ for every $t$ and Lemma 4.1 of [47] we have that:

$$
\left\|\mathrm{WP}_{\text {excited }}^{\epsilon}(\cdot, t)\right\|_{L^{2}}^{2}=\epsilon\left\|\mathfrak{a}_{-}^{0}(\cdot, t)\right\|_{L^{2}}^{2}\left\|\mathfrak{X}_{-}\left(\cdot ; \mathfrak{p}_{-}(t)\right)\right\|^{2}+o(\epsilon) .
$$

Using normalization of $\mathfrak{X}_{-}\left(\cdot ; \mathfrak{p}_{-}(t)\right)\left(\left\|\mathfrak{X}_{-}\left(\cdot ; \mathfrak{p}_{-}(t)\right)\right\|=1\right.$ for every $\left.t\right)$, and preservation of the $L_{y}^{2}$-norm by the equations defining $\mathfrak{a}_{-}(y, t)$ (recall Definition 3.10 and (2.14), we have that:

$$
\left\|\mathrm{WP}_{\text {excited }}^{\epsilon}(\cdot, t)\right\|_{L^{2}}^{2}=\epsilon\left\|a_{-}^{0}\left(\cdot, t^{*}\right)\right\|_{L^{2}}^{2}+o(\epsilon) .
$$

The precise form of $a_{-}^{0}\left(y, t^{*}\right)$ was derived in Section 5.1 and is displayed in (3.41). Taking the $L^{2}$-norm and squaring both sides of this expression we have:

$$
\begin{aligned}
& \left\|a_{-}^{0}\left(\cdot, t^{*}\right)\right\|_{L^{2}}^{2}=\left|\partial_{q} W\left(q^{*}\right)\right|^{2} \times\left|\left\langle\chi_{-}\left(\cdot ; p^{*}\right) \mid \partial_{p} \chi_{+}\left(\cdot ; p^{*}\right)\right\rangle\right|^{2} \\
& \times\left\|\int_{-\infty}^{\infty} e^{i\left[\partial_{q} W\left(q^{*}\right)\right]\left[\partial_{p} E_{+}\left(p^{*}\right)-\partial_{p} E_{-}\left(p^{*}\right)\right] \tau^{2} / 2} \times a^{0, *}\left(y-\left[\partial_{p} E_{+}\left(p^{*}\right)-\partial_{p} E_{-}\left(p^{*}\right)\right] \tau\right) \mathrm{d} \tau\right\|_{L_{y}^{2}}^{2} .
\end{aligned}
$$

By changing variables (recall Property 3.1 (A4) that $\left.\partial_{p} E_{+}\left(p^{*}\right)-\partial_{p} E_{-}\left(p^{*}\right) \neq 0\right)$ we can write the integral as a convolution:

$$
\begin{aligned}
& \left\|a_{-}^{0}\left(\cdot, t^{*}\right)\right\|_{L^{2}}^{2}=\frac{\left|\partial_{q} W\left(q^{*}\right)\right|^{2} \times\left|\left\langle\chi_{-}\left(\cdot ; p^{*}\right) \mid \partial_{p} \chi_{+}\left(\cdot ; p^{*}\right)\right\rangle\right|^{2}}{\left|\partial_{p} E_{+}\left(p^{*}\right)-\partial_{p} E_{-}\left(p^{*}\right)\right|^{2}} \\
& \times\left\|\int_{-\infty}^{\infty} e^{\frac{i\left[\partial_{q} W\left(q^{*}\right)\right] \tau^{2}}{2\left[\partial_{p} E_{+}\left(p^{*}\right)-\partial_{p} E_{-}\left(p^{*}\right)\right]}} \times a^{0, *}(y-\tau) \mathrm{d} \tau\right\|_{L_{y}^{2}}^{2}
\end{aligned}
$$

Applying Plancharel's theorem and then the convolution theorem yields that:

$$
\begin{aligned}
& \left\|a_{-}^{0}\left(\cdot, t^{*}\right)\right\|_{L^{2}}^{2}=\frac{\left|\partial_{q} W\left(q^{*}\right)\right|^{2} \times\left|\left\langle\chi_{-}\left(\cdot ; p^{*}\right) \mid \partial_{p} \chi_{+}\left(\cdot ; p^{*}\right)\right\rangle\right|^{2}}{\left|\partial_{p} E_{+}\left(p^{*}\right)-\partial_{p} E_{-}\left(p^{*}\right)\right|^{2}} \\
& \times\left\|\mathcal{F}_{\tau}\left\{e^{\frac{i\left[\partial_{q} W\left(q^{*}\right)\right] \tau^{2}}{2\left[\partial_{p} E+\left(p^{*}\right)-\partial_{p} E_{-}\left(p^{*}\right)\right]}}\right\}(\xi) \times \mathcal{F}_{y}\left\{a^{0, *}(y)\right\}(\xi)\right\|_{L_{\xi}^{2}}^{2},
\end{aligned}
$$

where $\mathcal{F}_{\tau}$ denotes the Fourier transform with respect to the variable $\tau$ :

$$
\mathcal{F}_{\tau}\{f(\tau)\}(\xi):=\int_{-\infty}^{\infty} e^{-2 \pi i \xi \tau} f(\tau) \mathrm{d} \tau
$$

A standard calculation shows that:

$$
\begin{aligned}
& \mathcal{F}_{\tau}\left\{e^{\frac{i\left[\partial_{q} W\left(q^{*}\right)\right] \tau^{2}}{2\left[\partial_{p} E_{+}\left(p^{*}\right)-\partial_{p} E_{-}\left(p^{*}\right)\right]}}\right\}(\xi)= \\
& \frac{\sqrt{\pi}(1+i) \sqrt{\left[\partial_{p} E_{+}\left(p^{*}\right)-\partial_{p} E_{-}\left(p^{*}\right)\right]}}{\sqrt{\left[\partial_{q} W\left(q^{*}\right)\right]}} e^{\frac{-i 2 \pi^{2}\left[\partial_{p} E_{+}\left(p^{*}\right)-\partial_{p} E_{-}\left(p^{*}\right)\right] \xi^{2}}{\left[\partial_{q} W\left(q^{*}\right)\right]}} .
\end{aligned}
$$

Substituting A.9 into A.7 and again using Plancharel's theorem yields:

$$
\left\|a_{-}^{0}\left(\cdot, t^{*}\right)\right\|_{L^{2}}^{2}=\frac{2 \pi\left|\partial_{q} W\left(q^{*}\right)\left\|\left.\left\langle\chi_{-}\left(\cdot ; p^{*}\right) \mid \partial_{p} \chi_{+}\left(\cdot ; p^{*}\right)\right\rangle\right|^{2}\right\| a^{0, *}(\cdot) \|_{L^{2}}^{2}\right.}{\left|\partial_{p} E_{+}\left(p^{*}\right)-\partial_{p} E_{-}\left(p^{*}\right)\right|} .
$$

Recall that $a^{0, *}(y)$ is defined as the limit: $a^{0, *}(y):=\lim _{t \uparrow t^{*}} a^{0}(y, t)$ where $a^{0}(y, t)$ is the leading-order envelope of the incident wavepacket and satisfies (2.14). By the $L_{y}^{2}$-norm conserving property of 2.14, we have that:

$$
\left\|a^{0, *}(\cdot)\right\|_{L^{2}}^{2}=\lim _{t \uparrow t^{*}}\left\|a^{0}(\cdot, t)\right\|_{L^{2}}=\left\|a^{0}(\cdot, 0)\right\|_{L^{2}}^{2}=\left\|a_{0}^{0}(\cdot)\right\|_{L^{2}}^{2}
$$


Substituting A.11 into A.10 we obtain:

$$
\left\|a_{-}^{0}\left(\cdot, t^{*}\right)\right\|_{L^{2}}^{2}=\frac{2 \pi\left|\partial_{q} W\left(q^{*}\right)\right|\left|\left\langle\chi_{-}\left(\cdot ; p^{*}\right) \mid \partial_{p} \chi_{+}\left(\cdot ; p^{*}\right)\right\rangle\right|^{2}\left\|a_{0}^{0}(\cdot)\right\|_{L^{2}}^{2}}{\left|\partial_{p} E_{+}\left(p^{*}\right)-\partial_{p} E_{-}\left(p^{*}\right)\right|} .
$$

Combining A.12 with A.4 we have that:

$$
\begin{aligned}
& \left\|\mathrm{WP}_{\text {excited }}^{\epsilon}(\cdot, t)\right\|_{L^{2}}^{2}= \\
& \quad \frac{2 \pi\left|\partial_{q} W\left(q^{*}\right)\left\|\left.\left\langle\chi_{-}\left(\cdot ; p^{*}\right) \mid \partial_{p} \chi_{+}\left(\cdot ; p^{*}\right)\right\rangle\right|^{2}\right\| a_{0}^{0}(\cdot) \|_{L^{2}}^{2}\right.}{\left|\partial_{p} E_{+}\left(p^{*}\right)-\partial_{p} E_{-}\left(p^{*}\right)\right|} \times \epsilon+o(\epsilon) .
\end{aligned}
$$

For the purposes of comparing our result with 'Landau-Zener' theory, we seek an expression for the magnitude of the excited wavepacket A.13 in terms of that of the initial wavepacket. First, recall that the initial wavepacket has the form (3.27):

$$
\mathrm{WP}_{\text {initial }}^{\epsilon}(x):=\mathrm{WP}^{1, \epsilon}\left[S_{0}, q_{0}, p_{0}, a_{0}^{0}(y), a_{0}^{1}(y), \chi_{n}\left(z ; p_{0}\right)\right](x) .
$$

By an identical calculation to that given above A.2)-A.4, we see that:

$$
\left\|\mathrm{WP}_{\text {initial }}^{\epsilon}(\cdot)\right\|_{L^{2}}^{2}=\left\|a_{0}^{0}(\cdot)\right\|_{L^{2}}^{2}+o(\sqrt{\epsilon}),
$$

and hence:

$$
\begin{aligned}
& \left\|\mathrm{WP}_{\text {excited }}^{\epsilon}(\cdot, t)\right\|_{L^{2}}^{2}= \\
& \quad \frac{2 \pi\left|\partial_{q} W\left(q^{*}\right) \|\left\langle\chi_{-}\left(\cdot ; p^{*}\right) \mid \partial_{p} \chi_{+}\left(\cdot ; p^{*}\right)\right\rangle\right|^{2}}{\left|\partial_{p} E_{+}\left(p^{*}\right)-\partial_{p} E_{-}\left(p^{*}\right)\right|} \times \epsilon \times\left\|\mathrm{WP}_{\text {initial }}^{\epsilon}(\cdot)\right\|_{L^{2}}^{2}+o(\epsilon) .
\end{aligned}
$$

Using $\dot{p}_{+}(t)=-\partial_{q} W\left(q_{+}(t)\right)$ 3.16, we may re-write A.16 in the equivalent form:

$$
\begin{aligned}
& \left\|\mathrm{WP}_{\text {excited }}^{\epsilon}(\cdot, t)\right\|_{L^{2}}^{2}= \\
& \left.\quad \frac{2 \pi\left|\left\langle\chi-\left(\cdot ; p_{+}(\tau)\right) \mid \frac{\mathrm{d}}{\mathrm{d} t} \chi_{+}\left(\cdot ; p_{+}(\tau)\right)\right\rangle\right|^{2}}{\left|\frac{\mathrm{d}}{\mathrm{d} \tau}\left(E_{+}\left(p_{+}(\tau)\right)-E_{-}\left(p_{+}(\tau)\right)\right)\right|}\right|_{\tau=t^{*}} \times \epsilon \times\left\|\mathrm{WP}_{\text {initial }}^{\epsilon}(\cdot)\right\|_{L^{2}}^{2}+o(\epsilon) .
\end{aligned}
$$

In the next subsection we shall compare the expression A.17 with A.31 of a Landau-Zener type model.

A.2. Agreement with 'Landau-Zener' theory. In this section we show that the expression (A.17) may be derived from a Landau-Zener type model, a simple finite dimensional model which incorporates key spectral features of the exact problem. We consider the following Schrödinger equation:

$$
i \epsilon \dot{\psi}^{\epsilon}=H(t) \psi^{\epsilon}, \quad t \geq 0 .
$$

Here, $H(t)$ denotes a time-dependent Hamiltonian whose spectrum consists of two time-dependent eigenvalue 'bands' which are degenerate at some $t^{*}>0$. Throughout this section, dots will denote derivatives with respect to $t$ hence $\dot{f}(t):=d f(t) / d t$. More precisely, we assume the following on the operator $H(t)$ :

$$
\operatorname{Spec}[H(t)]=E_{+}(t) \cup E_{-}(t),
$$

where $E_{+}(t), E_{-}(t)$ denote real, smooth functions which satisfy:

$$
\begin{aligned}
& E_{+}(t)<E_{-}(t) \text { for } t \in\left[0, t^{*}\right), \quad E_{-}(t)<E_{+}(t) \text { for } t>t^{*}, \\
& \text { and } E_{+}\left(t^{*}\right)=E_{-}\left(t^{*}\right) .
\end{aligned}
$$

We assume further that the crossing is linear in the sense that:

$$
\left.\left(\dot{E}_{+}(t)-\dot{E}_{-}(t)\right)\right|_{t=t^{*}}>0
$$


Let $\chi_{\sigma}(t), \sigma= \pm$ denote smoothly varying and orthonormal eigenfunctions corresponding to the eigenvalues $E_{\sigma}(t)$, so that:

$$
H(t) \chi_{\sigma}(t)=E_{\sigma}(t) \chi_{\sigma}(t), \quad t \geq 0,
$$

and $\left\langle\chi_{\sigma}(t) \mid \chi_{\sigma^{\prime}}(t)\right\rangle=\delta_{\sigma \sigma^{\prime}}$. We may assume without loss of generality (by making an appropriate gauge transformation) that:

$$
\left\langle\chi_{\sigma}(t) \mid \dot{\chi}_{\sigma}(t)\right\rangle=0, \quad t \geq 0 .
$$

This choice of gauge is sometimes known as the adiabatic gauge (see Remark 2.3). We now seek a solution of A.18 of the form:

$$
\psi^{\epsilon}(t)=\sum_{\sigma= \pm} c_{\sigma}(t) \chi_{\sigma}(t) e^{\frac{-i \int_{t^{*} E_{\sigma}(\tau) \mathrm{d} \tau}^{t}}{\epsilon}} .
$$

Substituting A.24 into A.18 and projecting onto the subspaces spanned by each eigenfunction yields a system for the coefficients $c_{\sigma}(t), \sigma= \pm$, which is equivalent to the original PDE (A.18):

$$
\begin{aligned}
\frac{\mathrm{d} c_{+}}{\mathrm{d} t}(t) & =\left\langle\chi_{+}(t) \mid \dot{\chi}_{-}(t)\right\rangle e^{\frac{i \int_{t^{*} E_{+}(\tau)-E_{-}(\tau) \mathrm{d} \tau}^{\epsilon}}{\mathrm{d}}} c_{-}(t) \\
\frac{\mathrm{d} c_{-}}{\mathrm{d} t}(t) & =\left\langle\chi_{-}(t) \mid \dot{\chi}_{+}(t)\right\rangle e^{\frac{i \int_{t^{*} E_{-}(\tau)-E_{+}(\tau) \mathrm{d} \tau}^{\epsilon}}{\epsilon}} c_{+}(t) .
\end{aligned}
$$

By differentiating $\left\langle\chi_{+}(t) \mid \chi_{-}(t)\right\rangle=0$ we obtain the identity:

$$
\left\langle\chi_{+}(t) \mid \dot{\chi}_{-}(t)\right\rangle=-\overline{\left\langle\chi_{-}(t) \mid \dot{\chi}_{+}(t)\right\rangle}, \quad t \geq 0 .
$$

We may then write the system A.25 in the compact form:

$$
\begin{aligned}
& \frac{\mathrm{d} c_{+}}{\mathrm{d} t}(t)=F^{\epsilon}(t) c_{-}(t), \quad \frac{\mathrm{d} c_{-}}{\mathrm{d} t}(t)=-\overline{F^{\epsilon}(t)} c_{+}(t), \\
& F^{\epsilon}(t):=-\overline{\left\langle\chi_{-}(t) \mid \dot{\chi}_{+}(t)\right\rangle} e^{\frac{i \int_{t^{*} E_{+}(\tau)-E_{-}(\tau) \mathrm{d} \tau}^{\epsilon}}{\epsilon}} .
\end{aligned}
$$

Integrating [A.27 in time gives:

$$
\begin{aligned}
& c_{+}(t)=c_{+}(0)+\int_{0}^{t} F^{\epsilon}(\tau) c_{-}(\tau) \mathrm{d} \tau \\
& c_{-}(t)=c_{-}(0)-\int_{0}^{t} \overline{F^{\epsilon}(\tau)} c_{+}(\tau) \mathrm{d} \tau .
\end{aligned}
$$

At this point, fix $t>t^{*}$. A standard calculation using oscillations in $F^{\epsilon}$ A.27 and the linear crossing assumption A.21 implies that:

$$
\begin{aligned}
& c_{+}(t)=c_{+}(0)-\left.\frac{\sqrt{\pi}(1+i) \overline{\left\langle\chi_{-}(\tau) \mid \dot{\chi}_{+}(\tau)\right\rangle} c_{-}(\tau)}{\sqrt{\left(\dot{E}_{+}(\tau)-\dot{E}_{-}(\tau)\right)}}\right|_{\tau=t^{*}} \times \sqrt{\epsilon}+o(\sqrt{\epsilon}) \\
& c_{-}(t)=c_{-}(0)+\left.\frac{\sqrt{\pi}(1-i)\left\langle\chi_{-}(\tau) \mid \dot{\chi}_{+}(\tau)\right\rangle c_{+}(\tau)}{\sqrt{\left(\dot{E}_{+}(\tau)-\dot{E}_{-}(\tau)\right)}}\right|_{\tau=t^{*}} \times \sqrt{\epsilon}+o(\sqrt{\epsilon}) .
\end{aligned}
$$


A similar calculation yields that $\left|c_{\sigma}\left(t^{*}\right)-c_{\sigma}(0)\right|=o(1), \sigma= \pm$, and hence:

$$
\begin{gathered}
c_{+}(t)=c_{+}(0)-\left.\frac{\sqrt{\pi}(1+i) \overline{\left\langle\chi_{-}(\tau) \mid \dot{\chi}_{+}(\tau)\right\rangle}}{\sqrt{\left(\dot{E}_{+}(\tau)-\dot{E}_{-}(\tau)\right)}}\right|_{\tau=t^{*}} \times \sqrt{\epsilon} \times c_{-}(0)+o(\sqrt{\epsilon}) \\
c_{-}(t)=c_{-}(0)+\left.\frac{\sqrt{\pi}(1-i)\left\langle\chi_{-}(\tau) \mid \dot{\chi}_{+}(\tau)\right\rangle}{\sqrt{\left(\dot{E}_{+}(\tau)-\dot{E}_{-}(\tau)\right)}}\right|_{\tau=t^{*}} \times \sqrt{\epsilon} \times c_{+}(0)+o(\sqrt{\epsilon}) .
\end{gathered}
$$

To compare with A.17), we now set $c_{-}(0)=0$ and square the absolute value of the expression for $c_{-}(t)$ to obtain:

$$
\left|c_{-}(t)\right|^{2}=\left.\frac{2 \pi\left|\left\langle\chi_{-}(\tau) \mid \dot{\chi}_{+}(\tau)\right\rangle\right|^{2}}{\left|\left(\dot{E}_{+}(\tau)-\dot{E}_{-}(\tau)\right)\right|}\right|_{\tau=t^{*}} \times \epsilon \times\left|c_{+}(0)\right|^{2}+o(\epsilon)
$$

When the initial data is normalized: $\left|c_{+}(0)\right|^{2}=1$, expressions A.31 and A.17 may be interpreted as the 'inter-band transition probability'.

\section{B. Proof that the 'INTER-BAND COUPLing COEFFICIENT' VANishes For TRIVIAL CROSSINGS}

B.1. Formula for $\left\langle\chi_{-}\left(\cdot ; p^{*}\right) \mid \partial_{p} \chi_{+}\left(\cdot ; p^{*}\right)\right\rangle$ from symmetry of Bloch band. Let $E(p), \chi(z ; p)$ denote an eigenpair of $(2.3)$. Then:

$$
\begin{aligned}
& H(2 \pi-p) e^{-2 \pi i z} \overline{\chi(z ; p)}=e^{-2 \pi i z} H(-p) \overline{\chi(z ; p)} \\
& =e^{-2 \pi i z} \overline{H(p) \chi(z ; p)}=E(p) e^{-2 \pi i z} \overline{\chi(z ; p)} \\
& e^{-2 \pi i(z+1)} \overline{\chi(z+1 ; p)}=e^{-2 \pi i z} \overline{\chi(z ; p)} .
\end{aligned}
$$

Hence, for $p \in \mathcal{B}$ such that the eigenvalue $E(p)$ is non-degenerate, $E(p)$ and $\chi(z ; p)$ obey the symmetry (after possibly multiplying $\chi(z ; p)$ by a constant):

$$
E(2 \pi-p)=E(p) \quad \chi(z ; 2 \pi-p)=e^{-2 \pi i z} \overline{\chi(z ; p)} .
$$

Note further that the symmetry $(\mathrm{B} .2)$ implies that the eigenvalue $E(2 \pi-p)$ is nondegenerate if and only if $E(p)$ is; if it were not, we could use $(\mathrm{B} .2)$ to generate two linearly independent eigenfunctions with eigenvalue $E(p)$ from those with eigenvalue $E(2 \pi-p)$.

Now, let $E_{n}(p), E_{n+1}(p)$ denote eigenvalue bands of 2.3 which cross at $p=\pi$ (Without loss of generality: the case where the crossing takes place at $p=0$ is similar and these are the only possibilities (Theorem 3.2p), and fix the Brillouin zone: $\mathcal{B}=[0,2 \pi]$. Let $E_{+}(p), E_{-}(p)$ and $\chi_{+}(z ; p), \chi_{-}(z ; p)$ denote the smooth eigenpairs defined in a neighborhood $U$ of $\pi$ by (3.3). It follows from (B.2) that for $p$ away from the degeneracy at $\pi, \chi_{+}(z ; p)$ and $\chi_{-}(z ; p)$ obey the symmetry:

$$
\chi_{-}(z ; p)=e^{-2 \pi i z} \overline{\chi_{+}(z ; p)}, \quad p \in U \backslash\{\pi\} .
$$

But now recall that the maps $\chi_{+}(z ; p), \chi_{-}(z ; p)$ are smooth at $p=\pi$, hence:

$$
\chi_{-}(z ; \pi)=\lim _{p \uparrow \pi} \chi_{-}(z ; p)=\lim _{p \uparrow \pi} e^{-2 \pi i z} \overline{\chi_{+}(z ; p)}=e^{-2 \pi i z} \overline{\chi_{+}(z ; \pi)} .
$$


It follows that (B.3) holds for every $p \in U$ :

$$
\chi_{-}(z ; p)=e^{-2 \pi i z} \overline{\chi_{+}(z ; p)}, \quad p \in U .
$$

Substituting B.5 into the formula for the 'inter-band coupling coefficient' 3.30 gives:

$$
\left\langle\chi_{-}(z ; \pi) \mid \partial_{p} \chi_{+}(z ; \pi)\right\rangle=\left\langle e^{-2 \pi i z} \overline{\chi_{+}(z ; p)} \mid \partial_{p} \chi_{+}(z ; \pi)\right\rangle=\int_{0}^{1} e^{2 \pi i z} \chi_{+}(z ; \pi) \partial_{p} \chi_{+}(z ; \pi) \mathrm{d} z .
$$

B.2. Proof that coefficient vanishes for trivial crossings. Now, suppose that $E_{n}(p)$ and $E_{n+1}(p)$ cross trivially in the sense that $V(z)=V_{1 / 2}(z)$, where $V_{1 / 2}(z)$ denotes a 1/2-periodic function, and the smooth band functions $E_{+}(p), E_{-}(p)$ and associated eigenfunctions $\chi_{+}(z ; p), \chi_{-}(z ; p)$ defined in a neighborhood of $p=\pi$ satisfy (all equality of eigenfunctions understood as holding up to a constant phase):

$$
\begin{array}{ll}
E_{+}(p)=\tilde{E}(p) & \chi_{+}(z ; p)=\tilde{\chi}(z ; p) \\
E_{-}(p)=\tilde{E}(2 \pi+p) & \chi_{-}(z ; p)=\tilde{\chi}(z ; 2 \pi+p)
\end{array}
$$

where $\tilde{E}(p)$ is an eigenvalue band of the Bloch eigenvalue problem $(2.3)$ with potential $V(z)=V_{1 / 2}(z)$ and $1 / 2$-periodic boundary conditions, considered on the Brillouin zone $[0,4 \pi]$ (see Figure 5).

B.7) in particular implies that $\chi_{+}(z ; p), \partial_{p} \chi_{+}(z ; p)$, and the function:

$$
\chi_{+}(z ; p) \partial_{p} \chi_{+}(z ; p) \text {, }
$$

are all $1 / 2$-periodic for all $p \in U$. It follows that the function (B.8) has for all $p \in U$ a convergent Fourier series with only even index modes:

$$
\begin{aligned}
& \chi_{+}(z ; p) \partial_{p} \chi_{+}(z ; p)=\sum_{m \in 2 \mathbb{Z}} c_{m} e^{2 \pi i m z}, \\
& c_{m}:=\int_{0}^{1} e^{-2 \pi i m z^{\prime}} \chi_{+}\left(z^{\prime} ; p\right) \partial_{p} \chi_{+}\left(z^{\prime} ; p\right) \mathrm{d} z^{\prime} .
\end{aligned}
$$

Substituting $(\overline{B .9}$ into $(\mathrm{B} .6)$ we have that:

$$
\left\langle\chi_{-}(z ; \pi) \mid \partial_{p} \chi_{+}(z ; \pi)\right\rangle=\sum_{m \in 2 \mathbb{Z}} c_{m} \int_{0}^{1} e^{2 \pi i z} e^{2 \pi i m z} \mathrm{~d} z,
$$

where the $c_{m}$ are as in $(\mathrm{B} .9)$. But:

$$
\int_{0}^{1} e^{2 \pi i(m+1) z} \mathrm{~d} z=0, \quad m \in 2 \mathbb{Z} .
$$

Hence $\left\langle\chi_{-}(z ; \pi) \mid \partial_{p} \chi_{+}(z ; \pi)\right\rangle=0$ as required.

\section{REFERENCES}

[1] N. I. Akhiezer. Elements of the theory of elliptic functions. Translations of Mathematical Monographs 79. American Mathematical Society, 1990.

[2] N.W. Ashcroft and N.D. Mermin. Solid State Physics. Saunders College, 1976.

[3] M. V. Berry. "Quantal phase factors accompanying adiabatic changes". In: Proceedings of the Royal Society of London A: Mathematical, Physical and Engineering Sciences. Vol. 392. 1802. The Royal Society. 1984, pp. 45-57. 
[4] G. Borg. "Eine Umkehrung der Sturm-Liouvilleschen Eigenwertaufgabe: Bestimmung der Differentialgleichung durch die Eigenwerte". In: Acta Mathematica 78 (1946), pp. 1-96.

[5] K. Cai. "Dispersive properties of Schrodinger equations". PhD thesis. California Institute of Technology, 2005.

[6] R. Carles and C. Sparber. "Semiclassical wave packet dynamics in Schrödinger equations with periodic potentials". In: Discrete Contin. Dyn. Syst. Ser. B 17.3 (2012), pp. 759-774.

[7] L. Chai, S. Jin, and Q. Li. "Semiclassical models for the Schrödinger equation with periodic potentials and band crossings". In: Kinetic and related models 6.3 (2013), pp. 505-532.

[8] L. Chai, S. Jin, Q. Li, and O. Morandi. "A multiband semiclassical model for surface hopping quantum mechanics". In: Multiscale Modeling 86 Simulation 13.1 (2015), pp. 205-230.

[9] K. S. Chandrasekharan. Elliptic functions. Grundlehren der Mathematischen Wissenschaften 281. Springer-Verlag Berlin, 1985.

[10] T. Duyckaerts, C. Fermanian-Kammerer, and T. Jecko. "Degenerated codimension 1 crossings and resolvent estimates". In: Asymptotic Analysis 65.3-4 (2009), pp. 147-174.

[11] M. S. P. Eastham. The spectral theory of periodic differential equations. Scottish Academic Press Ltd., London, 1973.

[12] C. Fefferman, J.P. Lee-Thorp, and M.I. Weinstein. "Edge states in honeycomb structures". In: Annals of PDE (to appear).

[13] C. Fefferman, J.P. Lee-Thorp, and M.I. Weinstein. "Honeycomb Schroedinger operators in the strong binding regime". In: Comm. Pure Appl. Math. (to appear).

[14] C. L. Fefferman, J. P. Lee-Thorp, and M. I. Weinstein. "Topologically protected states in one-dimensional continuous systems and Dirac points". In: Proceedings of the National Academy of Sciences 111.24 (2014), pp. 87598763.

[15] C. L. Fefferman, J. P. Lee-Thorp, and M. I. Weinstein. "Bifurcations of edge states - topologically protected and non-protected - in continuous 2D honeycomb structures". In: 2D Materials 3.1 (2016), p. 014008.

[16] C. L. Fefferman and M. I. Weinstein. "Honeycomb lattice potentials and Dirac points". In: Journal of the American Mathematical Society 25.4 (2012), pp. 1169-1220.

[17] C. L. Fefferman and M. I. Weinstein. "Wave packets in honeycomb structures and two-dimensional Dirac equations". In: Communications in Mathematical Physics 326.1 (2014), pp. 251-286.

[18] C. Fermanian-Kammerer and P. Gérard. "Mesures semi-classiques et croisement de modes". In: Bulletin de la Société Mathématique de France 130.1 (2002), pp. 123-168.

[19] C. Fermanian-Kammerer and P. Gérard. "A Landau-Zener formula for nondegenerated involutive codimension 3 crossings". In: Annales Henri Poincaré 4.3 (2003), pp. 513-552.

[20] C. Fermanian-Kammerer, P. Gérard, and C. Lasser. "Wigner measure propagation and conical singularity for general initial data". In: Archive for Rational Mechanics and Analysis 209.1 (2013), pp. 209-236. 
[21] C. Fermanian-Kammerer and C. Lasser. "Propagation through generic level crossings: a surface hopping semigroup". In: SIAM Journal on Mathematical Analysis 40.1 (2008), pp. 103-133.

[22] C. Fermanian-Kammerer and C. Lasser. "An Egorov theorem for avoided crossings of eigenvalue surfaces". In: Communications in Mathematical Physics 353.3 (2017), pp. 1011-1057.

[23] C. Fermanian-Kammerer and F. Mehats. "A kinetic model for the transport of electrons in a graphene layer". In: Journal of Computational Physics 327 (2016), pp. 450-483.

[24] P. Gérard. "Mesures semi-classiques et croisement de modes". In: Séminaire Équations aux dérivées partielles (Polytechnique) 16 (1990-1991), pp. 1-9.

[25] P. Gérard, P. A. Markowich, N. J. Mauser, and F. Poupaud. "Homogenization limits and Wigner transforms". In: Communications on Pure and Applied Mathematics 50.4 (1997), pp. 323-379.

[26] G. A. Hagedorn. "Proof of the Landau-Zener formula in an adiabatic limit with small eigenvalue gaps". In: Communications in Mathematical Physics 136.3 (1991), pp. 443-449.

[27] G. A. Hagedorn. Molecular propagation through electron energy level crossings. Vol. 536. Memoirs of the American Mathematical Society. American Mathematical Society, 1994.

[28] G. A. Hagedorn and A. Joye. "Landau-Zener transitions through small electronic eigenvalue gaps in the Born-Oppenheimer approximation". In: Annales de l'IHP Physique théorique. Vol. 68. 1. 1998, pp. 85-134.

[29] H. Hochstadt. "On the characterization of a Hill's equation from its spectrum". In: Archive for Rational Mechanics and Analysis 19 (1965), pp. 353611.

[30] H. Hochstadt. "On the determination of a Hill's equation from its spectrum II". In: Archive for Rational Mechanics and Analysis 23.3 (1966), pp. 237238.

[31] T. Jecko. "Semiclassical resolvent estimates for Schrödinger matrix operators with eigenvalues crossing". In: Mathematische Nachrichten 257.1 (2003), pp. $36-54$.

[32] A. Joye. "Proof of the Landau-Zener formula". In: Asymptotic Analysis 9.3 (1994), pp. 209-258.

[33] P. Kuchment. "An overview of periodic elliptic operators". In: Bulletin of the American Mathematical Society 53.3 (2016), pp. 343-414.

[34] L. Landau. "Zur Theorie der Energieübertragung. II". In: Physics of the Soviet Union 2 (1932), pp. 46-51.

[35] C. Lasser and S. Teufel. "Propagation through conical crossings: an asymptotic semigroup". In: Communications on Pure and Applied Analysis 58.9 (2005), pp. 1188-1230.

[36] W. Magnus and S. Winkler. Hill's Equation. John Wiley \& Sons, New York, 1966.

[37] H. Nakamura. Nonadiabatic transition: Concepts, Basic Theories and Applications. World Scientific Publishing Co. Pte. Ltd., 2002.

[38] J. Pöschel and E. Trubowitz. Inverse Spectral Theory. Pure and Applied Mathematics 130. Academic Press, 1987. 
[39] F. Poupaud and C. Ringhofer. "Semi-classical limits in a crystal with exterior potentials and effective mass theorems". In: Communications in Partial Differential Equations 21.11-12 (1996), pp. 1897-1918.

[40] M. Reed and B. Simon. Methods of modern mathematical physics, IV: analysis of operators. Academic press, 1977.

[41] R. Schubert, R. O. Vallejos, and F. Toscano. "How do wave packets spread? Time evolution on Ehrenfest time scales". In: Journal of Physics A: Mathematical and Theoretical 45.21 (2012), pp. 215-307.

[42] B. Simon. "On the genericity of nonvanishing instability intervals in Hills equation". In: Annales de l'I.H.P., Section A 24.1 (1976), pp. 91-93.

[43] Y. Colin de Verdiere. "The level crossing problem in semi-classical analysis I. The symmetric case". In: Annales de l'institut Fourier 53.4 (2003), pp. 10231054.

[44] Y. Colin de Verdiere. "The level crossing problem in semi-classical analysis II. The Hermitian case". In: Annales de l'institut Fourier 54.5 (2004), pp. 14231441.

[45] Y. Colin de Verdiere, M. Lombardi, and J. Pollet. "The microlocal LandauZener formula". In: Annales de l'I.H.P. Physique théorique 71.1 (1999), pp. 95127.

[46] A. B. Watson. "Wave dynamics in locally periodic structures by multiscale analysis". PhD thesis. https://doi.org/10.7916/D89W0SSM: Columbia University, 2017.

[47] A. B. Watson, J. Lu, and M. I. Weinstein. "Wavepackets in inhomogeneous periodic media: Effective particle-field dynamics and Berry curvature". In: Journal of Mathematical Physics 58.2 (2017), p. 021503.

[48] E. T. Whittaker and G. N. Watson. A Course of Modern Analysis. Cambridge University Press, 1902.

[49] C. Zener. "Non-adiabatic crossing of energy levels". In: Proceedings of the Royal Society of London A: Mathematical, Physical and Engineering Sciences 137.833 (1932), pp. 696-702. 